\title{
Dense gas in luminous infrared galaxies *
}

\author{
W. A. Baan ${ }^{1}$, C. Henkel ${ }^{2}$, A. F. Loenen ${ }^{3,1}$, A. Baudry ${ }^{4}$, and T. Wiklind ${ }^{5}$ \\ 1 ASTRON, Oude Hoogeveensedijk 4, 7991 PD Dwingeloo, The Netherlands \\ e-mail: baan@astron.nl \\ 2 Max-Planck-Institut für Radioastronomie, Auf dem Hügel 69, 53121 Bonn, Germany \\ Kapteyn Astronomical Institute, PO Box 800, 9700 AV Groningen, The Netherlands \\ 4 Observatoire de l'Université de Bordeaux, 33270 Floirac, France \\ 5 Onsala Space Observatory, 43900 Onsala, Sweden \& Space Telescope Science Institute, Baltimore, Maryland 21218, USA
}

Received 31 January 2007 / Accepted 16 October 2007

ABSTRACT

\begin{abstract}
Aims. Molecules that trace the high-density regions of the interstellar medium have been observed in (ultra-)luminous (far-)infrared galaxies, in order to initiate multiple-molecule multiple-transition studies to evaluate the physical and chemical environment of the nuclear medium and its response to the ongoing nuclear activity.

Methods. The $\mathrm{HCN}(1-0), \mathrm{HNC}(1-0), \mathrm{HCO}^{+}(1-0), \mathrm{CN}(1-0)$ and $\mathrm{CN}(2-1), \mathrm{CO}(2-1)$, and $\mathrm{CS}(3-2)$ transitions were observed in sources covering three decades of infrared luminosity including sources with known $\mathrm{OH}$ megamaser activity. The data for the molecules that trace the high-density regions were augmented with data available in the literature.

Results. The integrated emissions of high-density tracer molecules show a strong relation to the far-infrared luminosity. Ratios of integrated line luminosities were used for a first-order diagnosis of the integrated molecular environment of the evolving nuclear starbursts. Diagnostic diagrams display significant differentiation among the sources that relate to the initial conditions and the radiative excitation environment. Initial differentiation was introduced between the FUV radiation field in photon-dominated-regions and the $\mathrm{X}$-ray field in X-ray-dominated-regions. The galaxies displaying $\mathrm{OH}$ megamaser activity have line ratios typical of photon-dominated regions.
\end{abstract}

Key words. infrared: galaxies - ISM: molecules - radio line: galaxies - galaxies: active - galaxies: starburst - masers

\section{Introduction}

Large amounts of high-density molecular gas play a crucial role in the physics of (ultra-)luminous (far-)infrared galaxies ((U)LIRGs), giving rise to spectacular starbursts and possibly providing the fuel for the emergence of active galactic nuclei (AGN). A strong linear relation has been found between the far-infrared $\left(L_{\mathrm{FIR}}\right)$ and $\mathrm{HCN}$ luminosities, the latter being an indicator of the high density $\left(n\left(\mathrm{H}_{2}\right) \gtrsim 10^{4} \mathrm{~cm}^{-3}\right)$ component of the interstellar medium. This arises predominantly from the nuclear region and indicates a close relation to the nuclear starburst environment and the production of the FIR luminosity (Gao \& Solomon 2004a). The molecular gas contributes a significant fraction to the dynamical mass of the central regions of FIR galaxies (Henkel et al. 1991; Young \& Scoville 1991).

Emissions of molecules that trace the high-density regions in LIRGs and ULIRGs may serve to study the characteristics of the extreme environments in the nuclear regions of starburst and AGN nuclei. While much of the energy generation of FIR-luminous galaxies has traditionally been attributed to AGN activity using their optical signatures, the NIR and radio signatures suggest significant starburst contributions (Genzel et al. 1998; Baan \& Klöckner 2006). Circumnuclear star formation in the densest regions serves as a power source for the majority of ULIRGs such that they even mimic the presence of an AGN (Baan \& Klöckner 2006). The ULIRG population is also characterized by $\mathrm{OH}$ megamasers (MM) (Baan 1989;

$\star$ Appendices A and B are only available in electronic form at http://www . aanda. org
Henkel \& Wilson 1990) resulting from the amplification of the radio continuum by FIR-pumped foreground gas. Few $\mathrm{H}_{2} \mathrm{CO} \mathrm{MM}$ are also found among this population (Baan et al. 1993; Araya et al. 2004).

The observed molecular emissions from the high-density components could carry the signature of the nuclear region of the ULIRG and its nuclear power generation. Recent studies consider the $\mathrm{HCN}(1-0)$ versus $\mathrm{CO}(1-0)$ relation and its implications for star formation activity (Gao \& Solomon 2004a,b). Discussions are ongoing in the literature about whether such molecular emissions are accurate tracers of the high-density medium at the site of star formation (Wu et al. 2005; Graciá-Carpio et al. 2006; Papadopoulos 2007). It should be evident that only multi-transition and multi-molecule (multidimensional) studies can describe the multi-parameter environment of the nuclear activity and further establish the connection between the molecular signature and the nature of the nuclear energy source. The translation or extrapolation from the characteristics of individual star formation regions in our Galaxy to integrated regions in nearby galaxies at larger distances or even to unresolved nuclear regions in distant galaxies requires detailed multi-species observations and comparisons combined with elaborate theoretical modelling. The interpretation of molecular line data has focused on the nature of the local excitation mechanisms in the form of photon dominated regions (PDR) with far-ultraviolet radiation fields and X-ray dominated regions (XDR) (Lepp \& Dalgarno 1996; Meijerink \& Spaans 2005). The molecular properties of these two different regimes can be used as diagnostic tools in interpreting the integrated properties of galaxies. 
This paper reflects a multi-dimensional approach to understanding molecular line emissions that started in the mid 90's, but is only now coming to fruition. This study originally aimed to establish a better link between molecular megamaser activity and the molecular properties of luminous FIR galaxies, but it has changed into a general study of ULIRGs. Here we present data on the molecular characteristics of normal and FIR galaxies across the available range of $L_{\mathrm{FIR}}$. Data of the CO, HCN, HNC, $\mathrm{HCO}^{+}, \mathrm{CN}$, and $\mathrm{CS}$ line emissions obtained for a representative group of 37 FIR-luminous and $\mathrm{OH}$ megamaser galaxies has been joined with the additional data of 80 sources presented in the literature. Early studies of the properties of CS (Mauersberger et al. 1989), $\mathrm{HCO}^{+}$(Nguyen-Q-Rieu et al. 1992), and HNC (Hüttemeister et al. 1995) using small numbers of sources studied the relations between line and FIR luminosities and the nature of the central engine. Our molecular emission data show clear tendencies that cover a wide regime of nuclear activity. This database forms the basis for a first evaluation of the emission line properties and for further study and modelling of the line properties.

\section{Characteristics of high-density tracers in galaxies}

Extragalactic line ratios do not display the extreme ratios found in the Galaxy, because they are integrations of ensembles of different types of clouds within one beam. Nevertheless, clear variations reflect the response of the molecular emission to the varying physical and chemical environments characterizing the regions of nuclear activity.

HCN and HNC molecules - Steady-state excitation models show that the abundance of HCN and its isotopomer HNC decreases with increasing density and temperature (Schilke et al. 1992). Values for the $\mathrm{HNC} / \mathrm{HCN}$ abundance ratio below unity are consistent with steady-state models for higher-density gas at higher temperatures. An $\mathrm{HNC} / \mathrm{HCN}$ abundance ratio greater than unity suggests a rather quiescent, low temperature gas. In dark clouds this ratio can be more than 3.0 (Churchwell et al. 1984) but in warm environments in giant molecular clouds, the ratio may decrease to values of 0.5 to 0.2 (Irvine et al. 1987). In the Orion Hot Core the observed HNC/HCN column density ratio can be as low as 0.013 with values of 0.2 on the ridge (Schilke et al. 1992). The observed $\mathrm{HNC} / \mathrm{HCN}$ intensity ratio is found to vary significantly from 0.16 to 2 among galaxies with similar $\mathrm{HCN} / \mathrm{CO}$ intensity ratios (Aalto et al. 2002), which may be connected with IR pumping (Aalto et al. 2007). This suggests that $\mathrm{HNC}$ is not reliable as a tracer of cold $(10 \mathrm{~K})$ high-density gas in LIR galaxies, as it is in the Galaxy. HCN has thus been used to determine the gas masses and the related star formation efficiencies (Gao \& Solomon 2004b).

Our exploratory LVG simulations suggest that, for a given density, column density, and temperature, $\mathrm{HCN}(1-0)$ and $\mathrm{HNC}(1-0)$ have similar intensities, but that $\mathrm{HCO}^{+}(1-0)$ is weaker. At higher temperatures HNC tends to be selectively destroyed in favor of $\mathrm{HCN}$ as long as the medium is not highly ionized. The canonical $\mathrm{HNC} / \mathrm{HCN}$ abundance ratio is 0.9 , if the standard neutral production paths are used (Goldsmith et al. 1981). In a highly ionized medium, low $\mathrm{HNC} / \mathrm{HCN}$ ratios would be prevented since $\mathrm{HCNH}^{+}$can form $\mathrm{HCN}$ and $\mathrm{HNC}$ with equal probability (Aalto et al. 2002; Wang et al. 2004). The passage of shocks may selectively destroy HNC and could significantly reduce the steady-state $\mathrm{HNC} / \mathrm{HCN}$ abundance ratio (Schilke et al. 1992).
The $\mathrm{HCO}^{+}$molecule - Hüttemeister et al. (1995) suggest a simplified picture where the molecules trace different gas components: $\mathrm{HCN}$ in the warm and dense part, $\mathrm{HNC}$ in the cool and slightly less dense part, and $\mathrm{HCO}^{+}$in the slightly less dense part, that is penetrated by cosmic rays and/or UV photons. The $\mathrm{HCN} / \mathrm{HCO}^{+}$intensity ratio may vary from 0.5 to $\geq 4$, which indicates that the molecules probe distinct physical regions in nearby galaxies (Nguyen-Q-Rieu et al. 1992). Alternatively, there have to be large-scale abundance differences and/or different types of chemistry. The excitation of $\mathrm{HCN}$ and $\mathrm{HCO}^{+}$in these galaxies suggests low optical depth of less than unity and very low excitation temperatures of $\leq 10 \mathrm{~K}$ (Nguyen-Q-Rieu et al. 1992). This conclusion is confirmed by studies of many species in NGC 4945 and other nearby galaxies (e.g., Wang et al. 2004). A multi-transition study of the high-density medium in Arp 220 and NGC 6240 suggests that HCN would serve better as a tracer of dense gas than $\mathrm{HCO}^{+}$, which can be more sub-thermally excited and as a radical avoids high densities (Greve et al. 2006).

Shock propagation can strongly change the gas-phase chemistry such that $\mathrm{HCO}^{+}$is enhanced relatively to $\mathrm{HCN}$ in gas associated with young supernova remnants (Dickinson et al. 1980; Wootten 1981). The shocked region of IC 443 shows an enhancement of two orders of magnitude in $\mathrm{HCO}^{+}$relative to $\mathrm{CO}$ (Dickinson et al. 1980). This enhancement of $\mathrm{HCO}^{+}$ would result from increased ionization due to cosmic rays in the shocked dense material (Wootten 1981; Elitzur 1983).

The CN molecule - The abundance of the cyanid radical $\mathrm{CN}$ is enhanced in the outer regions of molecular clouds in PDR dominated environments (Greaves \& Church 1996; Rodriguez-Franco et al. 1998). At larger depths the CN abundance declines rapidly (Jansen et al. 1995b). To a significant extent $\mathrm{CN}$ is a photo-dissociation product of $\mathrm{HCN}$ and HNC; e.g., the $\mathrm{CN} / \mathrm{HCN}$ abundance increases in the outer regions of molecular clouds that are exposed to ultraviolet (FUV) radiation (see Rodriguez-Franco et al. 1998). Therefore, the CN/HCN (1-0) line intensity ratios may be used to probe the physical and chemical conditions for Galactic and extragalactic PDR sources.

In the Orion bar the $\mathrm{CN}$ abundance is enhanced at the inner edge of the PDR, but it declines at larger depths (Jansen et al. 1995a). The Orion-KL region shows HCN (1-0) emission that is significantly stronger than the HNC and CN (1-0) emission, which is predicted for warm $(T \gtrsim 50 \mathrm{~K})$ gas (Ungerechts et al. 1997).

Using $\mathrm{CN}(1-0)$ and $\mathrm{CN}(2-1)$, as well as $\mathrm{HCN}(1-0)$ measurements across the inner star-forming molecular disk in $\mathrm{M} 82$, Fuente et al. (2005) find that $N_{\mathrm{CN}} / N_{\mathrm{HCN}} \approx 5$. They argue that such a high ratio is indicative of a giant and dense PDR bathed in the intense radiation field of the starburst and conclude that $A_{\mathrm{V}} \leq 5$ in the clouds of M82, because for optically thicker clouds the $\mathrm{CN} / \mathrm{HCN}$ column density ratio would be smaller than observed. The effect of enhanced $\mathrm{CN} / \mathrm{HCN}$ abundance and line temperature ratios is confirmed by theoretical models of $\mathrm{CN}$ and HCN gas-phase chemistry (Boger \& Sternberg 2005). This suggests that the $\mathrm{CN} / \mathrm{HCN}$ abundances ratio in molecular clouds may be used as probes of FUV and cosmic-ray driven gas-phase chemistry, for a wide range of conditions. Boger \& Sternberg (2005) find that in dense gas, CN molecules are characteristically and preferentially produced near the inner edges of the C II zones in the PDRs. In addition, chemical models (Krolik \& Kallman 1983; Lepp \& Dalgarno 1996) indicate an enhancement of $\mathrm{CN}$ under high X-ray ionization conditions. The prominence of $\mathrm{HNC}$ and $\mathrm{CN}$ relative to $\mathrm{HCN}$ has been considered a measure of the evolutionary stage of the respective starburst (Aalto et al. 2002). This way the objects with strong HCN and weak HNC 
and $\mathrm{CN}$ are dominated by a warm dense gas environment early in the evolution.

The CS molecule - Multi-transition studies of CS in selected Galactic clouds by Snell et al. (1984) show that there is considerable density variation across their core regions, because the lower (column) density outer regions contribute little to the total CS emission. The cloud core is approximately two orders of magnitude denser than the extended cloud, and the density rises very steeply towards the core. LVG-modelling of the data suggests a mean density of 5 to $10 \times 10^{5} \mathrm{~cm}^{-3}$ with cloud core masses ranging from $3 \times 10^{5}$ to $2 \times 10^{3} M_{\odot}$. The CS abundance varies considerably, peaking in the clumped high-density medium. Chemical studies suggest that the fractional abundance of CS is sensitive to both the abundances of sulfur and oxygen (Graedel et al. 1982).

Although the CS lines may be brightest toward star-forming cores in regions of high volume-density, ubiquitous low-level CS emission, probably due to sub-thermally excited low-density gas, is found to dominate the emission from the inner Galaxy (McQuinn et al. 2002) and its central region (Bally et al. 1988). On the other hand, the study of the excitation state of CS in the integrated nuclear environment of Arp 220 and NGC 6240 suggests that CS probes the densest gas in the high-density medium better than $\mathrm{HCN}$ or $\mathrm{HCO}^{+}$(Greve et al. 2006).

\section{Observations}

\subsection{IRAM Pico Veleta (PV) observations}

Observations with the 30-m IRAM ${ }^{1}$ telescope at Pico Veleta (PV) (Spain) were carried out in Sept. 1994, May 1996, Jan. 1997, and May 1997. At the frequencies of the $\lambda \sim 3.4 \mathrm{~mm}$ $J=1-0$ transitions of $\mathrm{HCN}, \mathrm{HNC}$, and $\mathrm{HCO}^{+}$the full width to half power beam size was $25^{\prime \prime}$; for the $\lambda=2.6$ and $1.3 \mathrm{~mm}$ CO $J=1-0$ and 2-1 transitions it was 21 and $13^{\prime \prime}$. Typically, observations were started with the $\mathrm{CO} J=1-0$ and 2-1 lines, then the lower frequency frontend was tuned to $\mathrm{HCN}, \mathrm{HNC}$ or $\mathrm{HCO}^{+}$while keeping $\mathrm{CO}(2-1)$ as a sensitive indicator of pointing quality.

During the first session in 1994, the $3 \mathrm{~mm}$ SIS receiver had a $512 \mathrm{MHz}$ bandwidth and a receiver temperature of 80-120 K below $100 \mathrm{GHz}$ (image sideband rejection 5-10 dB). Starting in 1996, the renewed SIS receivers were singlesideband tuned with image sideband rejections of 25-40 dB. Receiver temperatures were $T_{\text {rec }}(\mathrm{SSB})=55-85 \mathrm{~K}$ at a wavelength of $3.4 \mathrm{~mm}, 100-140 \mathrm{~K}$ at $2.6 \mathrm{~mm}, 130 \mathrm{~K}$ at $2.0 \mathrm{~mm}$, and $100-180 \mathrm{~K}$ at $1.3 \mathrm{~mm}$. The observations were done under varied atmospheric conditions, which were best for the runs in 1997. The system temperatures during these sessions were $200-300 \mathrm{~K}$ for the $80-90 \mathrm{GHz}$ range, $260-400 \mathrm{~K}$ around $150 \mathrm{GHz}$, and $700-1200 \mathrm{~K}$ around $230 \mathrm{GHz}$.

During the first session in 1994 we used the filterbank backend (FB) in a $512 \times 1 \mathrm{MHz}$ mode and the acousto-optical spectrometer (AOS) in a $512 \times 1 \mathrm{MHz}$ mode. During the 1996 and 1997 observations we used the AOS in $512 \times 2 \mathrm{MHz}$ mode in parallel with the FB in $256 \times 1 \mathrm{MHz}$ mode. The spectral resolution of the observations with $1 \mathrm{MHz}$ channels was $3.2 \mathrm{~km} \mathrm{~s}^{-1}$ at $115 \mathrm{GHz}$ and $1.6 \mathrm{~km} \mathrm{~s}^{-1}$ at $230 \mathrm{GHz}$.

The observing procedure included the use of the wobbling secondary mirror in order to minimize baseline problems, which allowed switching between source and reference positions

1 Institute de Radioastronomie Millimétrique is supported by INSU/CNRS (France), the MPG (Germany), and the IGN (Spain). placed symmetrically at offsets of $4^{\prime}$ in azimuth. Each wobbler phase had a duration of $2 \mathrm{~s}$. From continuum cross scans through nearby pointing sources, we estimate the absolute pointing uncertainty to be of $\lesssim 3^{\prime \prime}$. The positional alignment between the receivers is estimated to be better than $2^{\prime \prime}$. For all sources single nuclear pointings were used.

The results of our observations are presented in units of main beam brightness temperature $\left(T_{\mathrm{mb}}\right.$; e.g. Downes 1989$)$. Using beam efficiencies of $0.78,0.75,0.69$, and 0.52 , we find main beam sensitivities of $S / T_{\mathrm{mb}}=4.86,4.72,4.62$, and $4.44 \mathrm{Jy} / \mathrm{K}$ at $\lambda \sim 3.4,2.6,2.0$, and $1.3 \mathrm{~mm}$. The calibration is affected by the pointing, baseline stability, and receiver stability from session to session. The overall accuracy of the data is estimated to be $15 \%$. Stability of calibration between the four observing periods has been ensured by using the same calibration sources during all of the sessions. All data has been calibrated using the standard chopper wheel method.

\subsection{SEST observations}

Two observing sessions were done at the Swedish/ESO ${ }^{2}$ Submillimeter Telescope (SEST) at La Silla (Chile) during January 1995 and January 1997. System performance during the first session prevented detection with a sufficient signal-to-noise ratio for the high-density lines of all our target sources except for IR07160-6215. The data presented in this paper have been predominantly obtained during the successful second session.

The observations were done using two Low-Resolution AOS systems with $1 \mathrm{GHz}$ bandwidth having a total of 1440 channels for a single polarization. The channel spacing was $1.4 \mathrm{MHz}$, corresponding to 5.2 and $3.6 \mathrm{~km} \mathrm{~s}^{-1}$ at 80 and $115 \mathrm{GHz}$. In 1997 the receiver temperatures were $T_{\text {rec }}(\mathrm{SSB})=100-180 \mathrm{~K}$ and $120-140 \mathrm{~K}$ in the $80-116 \mathrm{GHz}$ and $130-150 \mathrm{GHz}$ frequency ranges, respectively. Observations were conducted under very favorable atmospheric conditions and the actual system temperatures were between 150 and $200 \mathrm{~K}$ in the $80-90 \mathrm{GHz}$ band and between 250 and $350 \mathrm{~K}$ around $150 \mathrm{GHz}$.

The target transitions for the observations were the $\mathrm{HCN}(1-0)$ and $\mathrm{HCO}^{+}(1-0)$ transitions, which occur in a single bandpass, and the HNC(1-0) transition. Parallel observations were done of $\mathrm{CS}(3-2)$ using the other polarization. $\mathrm{CO}(1-0)$ observations were done for sources without known earlier detections. Single nuclear pointings were used for all sources. Pointing observations indicate that the absolute pointing uncertainty is of the order of $\lesssim 3^{\prime \prime}$.

Calibration was done with a chopper wheel and using the $T_{\mathrm{A}}^{*}$ intensity scale. The spectral data has been converted into a main-beam brightness temperature $T_{\mathrm{mb}}$, using a main-beam efficiency of 0.75 at $86 \mathrm{GHz}, 0.70$ at $115 \mathrm{GHz}$ and 0.60 at $150 \mathrm{GHz}$. Conversion factors to flux units are $18.75,18.90$, and $20.50 \mathrm{Jy} / \mathrm{K}$. The beam size is respectively $57^{\prime \prime}, 45^{\prime \prime}$, and $34^{\prime \prime}$.

\section{Results}

The observational results have been presented in Online Figs. A.1 and A.2 and in Tables B.1 and B.2. In the following evaluations, the luminosity distance $D_{\mathrm{L}}$ has been taken from the literature for sources with radial velocities below about $1500 \mathrm{~km} \mathrm{~s}^{-1}$ and determined from known emission line velocities or from NED using $H_{0}=72 \mathrm{~km} \mathrm{~s}^{-1}$ and $q_{0}=0.5$. The FIR luminosity $L_{\mathrm{FIR}}$ has been determined from the 60 and

\footnotetext{
${ }^{2}$ The European Southern Observatory.
} 
$100 \mu \mathrm{m}$ IRAS fluxes using an IR-color correction factor 1.8 (Sanders \& Mirabel 1996; Solomon et al. 1997).

IRAM Pico Veleta results - Our Pico Veleta 30-m observations of 18 luminous FIR sources have resulted in 66 detections of emission lines for the following transitions: 18 for $\mathrm{CO}(2-1)$, 11 for $\mathrm{HCN}(1-0), 8$ for $\mathrm{HNC}(1-0), 11$ for $\mathrm{HCO}^{+}(1-0), 8$ for $\mathrm{CS}(3-2), 6$ for $\mathrm{CN}(1-0)$ and 4 for $\mathrm{CN}(2-1)$. The target sample for $\mathrm{HCN}, \mathrm{HNC}$, and $\mathrm{HCO}^{+}$was selected on the basis of FIR luminosity, the presence of $\mathrm{OH}$ MM activity, or the strength of the CO $J=1-0$ line obtained earlier with the 12-m NRAO telescope (Baan \& Freund 1992, unpublished). Sources for observations of the cyano radical $\mathrm{CN}$ were selected on the basis of the strength of the $\mathrm{CO}$ and $\mathrm{HCN}$ lines. The $\mathrm{CS}(3-2)$ data has been obtained in parallel to the observations at lower frequencies. High-density tracer transitions in sources with published detections at the time of the observations were not re-observed. Individual spectra toward the nuclear positions obtained during our observations are presented in $T_{\mathrm{mb}}$ units in Figs. A.1 and A.2. Results from Gaussian fitting are presented in Table B.1 after conversion into units of $\mathrm{Jy}_{\mathrm{km} \mathrm{s}}{ }^{-1}$.

SEST results - The observations of 20 sources with the SEST 15-m telescope have resulted in a total of 52 new detections of emission lines in 16 sources for the following transitions: 12 for $\mathrm{CO}(1-0), 11$ for $\mathrm{HCN}(1-0), 8$ for $\mathrm{HNC}(1-0)$, 13 for $\mathrm{HCO}^{+}(1-0), 7$ for $\mathrm{CS}(3-2)$, and 1 for $\mathrm{CN}(1-0)$. Target sources during these sessions have been selected on the basis of the strength of known CO emission lines (Mirabel et al. 1990; Garay et al. 1993), and of the FIR luminosity taken from the IRAS catalogue. The source NGC 660 is common between the Pico Veleta and SEST data sets. High-density tracer lines in sources with published detections at the time of the observations were not re-observed. Individual spectra toward the nuclear positions are presented in Fig. A. 3 in $T_{\mathrm{mb}}$ units and the results from Gaussian fits are given in Table B.1 after conversion into units of $\mathrm{Jy} \mathrm{km} \mathrm{s}^{-1}$. Target sources without any line detection have been omitted from the tables.

Detections from the literature - The detections of our combined sample has been augmented with detections of molecular lines tracing the high-density component reported in the literature. In addition, we have used these detections to verify and cross-calibrate our own detections where possible. Thus we obtained detections of at least one of the $\operatorname{HCN}(1-0), \operatorname{HNC}(1-0)$, $\mathrm{HCO}^{+}(1-0), \mathrm{CN} N=1-0$ and $N=2-1$, and CS(3-2) transitions for a total of 80 additional sources. A total of 167 individual published records have been used, which include a significant number of HCN detections from Gao \& Solomon (2004a).

The integrated source sample - Our own sample of 37 sources has been augmented with the data of 80 sources from the literature. Table B.1 presents the available data on molecules that trace low-density and high-density components of the medium. The database contains a total number of 202 reliable detections of high-density tracers, and 110 for $\mathrm{CO}(1-0)$ with 32 accompanying $\mathrm{CO}(2-1)$ line detections. This represents an incomplete record for many of the sources. The database has 84 detections plus 18 upper limits for $\mathrm{HCN}, 28+13$ for $\mathrm{HNC}$, $42+10$ for $\mathrm{HCO}^{+}, 19+6$ for $\mathrm{CN}(1-0), 13+7$ for $\mathrm{CN}(2-1)$, and $17+20$ for $\mathrm{CS}(3-2)$. For 23 sources a complete set of $\mathrm{HCN}(1-0), \mathrm{HNC}(1-0)$ and $\mathrm{HCO}^{+}(1-0)$ tracer transitions were obtained and 10 sources have one out of three as an upper limit.

All data records for our integrated sample have been converted from $T_{\mathrm{mb}}$ into units of Jy $\mathrm{km} \mathrm{s}^{-1}$ for the PV and SEST results or from antenna temperature for other telescopes. We also note that some data in the literature has been obtained with various single-dish instruments: the 30-m Pico Veleta telescope, the 15-m SEST telescope, the 12-m NRAO telescope at Kitt Peak, the Onsala 20-m telescope, and the 14-m FCRAO telescope. The inclusion and comparison of this data has been complicated by the incomplete reporting of observing and calibration procedures. In many cases the $\mathrm{CO}(1-0)$ and $\mathrm{HCN}(1-0)$ line strengths from different data records have been used. General agreement was found for line data from multiple source records with errors within 30\%. Some published line data records, particularly in Gao \& Solomon (2004a), were not compatible with our data and other available data and could not be used in the compilation. As a result some line ratios are also different from those in other publications because of inconsistencies between our own data and some published data. The source NGC 660 was included in both our observing samples and a comparison of the $\mathrm{HCN}$ and $\mathrm{HCO}^{+}$lines shows agreement within $10 \%$. We also note that some of our own lower quality spectra and their unreliable fits have not been used in the evaluation and have been designated as upper limits in our data base in Tables 1 and 2 .

Evaluating the data - The collected data has been obtained at different observing beams and different beam filling factors. For a given telescope, the beam sizes at the observing frequencies of $\mathrm{HCN}, \mathrm{HNC}$, and $\mathrm{HCO}^{+}$are very similar and they differ by some $25 \%$ from those of $\mathrm{CN}(1-0)$ and $\mathrm{CO}(1-0)$. Since the emission of the high-density tracers is well-confined to the nuclear regions of the galaxies, it will generally not fill the beam. On the other hand, the $\mathrm{CO}(1-0)$ emission and possibly also the $\mathrm{CO}(2-1)$ emission will be more extended and could fill the beam for nearby galaxies. The extent of a typical CO (1-0) emission region in ULIRGs varies between 2 and $3 \mathrm{kpc}$ (Downes \& Solomon 1998); this would result in an underestimate of the $\mathrm{CO}$ emission at radial velocities less than $2000 \mathrm{~km} \mathrm{~s}^{-1}$ for the PV telescope and less than $1000 \mathrm{~km} \mathrm{~s}^{-1}$ for the SEST telescope.

Because of their similar frequencies, the line ratios of the high-density tracers are not affected by different beam filling factors. Their ratios with $\mathrm{CO}(1-0)$ would only be affected by an underestimate of the total $\mathrm{CO}$ emission. The line intensity ratio between $\mathrm{CO}(1-0)$ and the high density tracers used throughout this paper will therefore be representative of more distant galaxies, while they could be lower limits for nearby galaxies. We will return to the effect of relative filling factors in the evaluation of the CO line ratios below (Sect. 5.2).

\section{The $\mathrm{CO}$ line characteristics}

\subsection{Line luminosities}

The $J=1-0$ and 2-1 lines of CO observed by us are easily saturated and thermalized, such that they are likely not tracing the high-density regions $\left(n\left(\mathrm{H}_{2}\right) \geq 10^{4} \mathrm{~cm}^{-3}\right)$ of the ISM where the star formation occurs. The $\mathrm{CO}$ characteristics of our sample sources are shown in Fig. 1. Slopes (indicated in the figures with $s$ ) have been fitted to the data using a standard Least Squares Fit method (see Bevington \& Robinson 1992). To evaluate the significance of the fits, the correlation coefficient (indicated by $r$ ) has also been calculated. For these calculations upper limits have been omitted, and all data have been given the same statistical weight, since only few data points have known errors. Both CO transitions display a clear dependence on the FIR luminosity with slopes of $0.74 \pm 0.04$ (110 sources; $r=0.87$ ) and $0.91 \pm 0.10$ (32 sources; $r=0.85$ ). At high luminosities the scatter in $L_{\mathrm{CO}}$ is a factor 4 but increases at lower luminosities to a factor of 10 . This scatter can be attributed to variations of the star formation efficiency (SFE) $L_{\mathrm{FIR}} / M\left(\mathrm{H}_{2}\right)$, ranging between 1 and $100 L_{\odot} / M_{\odot}$, and (in part) to underestimates of the 

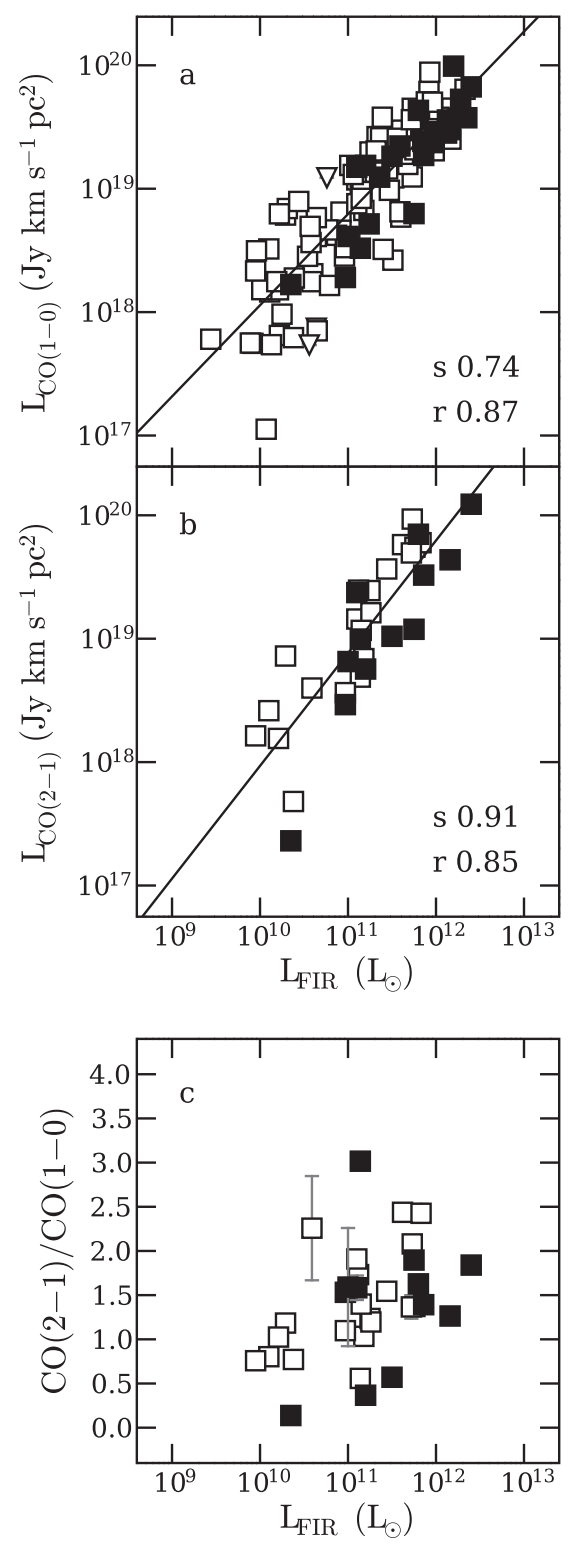

Fig. 1. Integrated line luminosities versus FIR luminosity for the integrated sample of sources. a) $\mathrm{CO}(1-0)$ luminosity versus FIR luminosity, b) $\mathrm{CO}(2-1)$ luminosity versus FIR luminosity, and c) $\mathrm{CO}(2-1) / \mathrm{CO}(1-0)$ luminosity ratio versus FIR luminosity. There are 110 reliable data points for $\mathrm{CO}(1-0)$ and 32 for $\mathrm{CO}(2-1)$. The fitted slope $(s)$ and the correlation coefficient $(r)$ are given in the lower right corner of each frame and the fit does not take into account any displayed upper limits. The data points in the diagrams are either squares for reliable values or triangles for upper and lower limits. Filled symbols indicate the $24 \mathrm{OH}$ or $\mathrm{H}_{2} \mathrm{CO} \mathrm{MM}$ sources in the sample. Open symbols may also represent undetected $\mathrm{OH}$ MM because of beaming effects or weak maser action with similar ISM conditions.

CO luminosity for the lower luminosity (and nearby) sources, where single-dish instruments only sample the central region.

The slopes for both $\mathrm{CO}$ curves are significantly less steep than unity, possibly related to optical depth effects or to an increase in the star formation efficiency SFE at high $L_{\mathrm{FIR}}$. Assuming constant $L_{\mathrm{CO}}$-to- $M\left(\mathrm{H}_{2}\right)$ conversions, earlier studies suggested a linear $L_{\mathrm{FIR}}-M\left(\mathrm{H}_{2}\right)$ relation with a slope that steepens at high $L_{\text {FIR }}$ due to a strong variation of the SFE (Young \& Scoville 1991). Similarly Braine \& Combes (1992) present a linear relation between the $\mathrm{CO}(2-1)$ and FIR data for $L_{\mathrm{FIR}} \leq 10^{10.5} L_{\odot}$. The relation with slope less than unity deduced from our data for $L_{\mathrm{FIR}} \geq 10^{10} L_{\odot}$ is consistent with characteristics of the upper luminosity range of the earlier data. Our fit would suggest SFE $\propto L_{\mathrm{FIR}}{ }^{0.25}$ for the higher end of the $L_{\mathrm{FIR}}$ distribution, or an equivalent variation in the $L_{\mathrm{CO}}$-to- $M\left(\mathrm{H}_{2}\right)$ conversion factor.

\subsection{The CO line ratio}

The $I_{\mathrm{CO}}(2-1) / I_{\mathrm{CO}}(1-0)$ line ratio has been displayed for 32 sources of which five have values greater than $2(\mathrm{OH} M M$ IRAS 15107+0724, NGC 660, NGC 3256, IRAS 06259-4708, and IRAS 18293-3413; Fig. 1c). Earlier results from nearby spiral galaxies (Radford et al. 1991; Braine \& Combes 1992) as well as the data from this study show a concentration of data points around 1.5 with a few outliers up to 3 .

An observed line ratio of $\geq 1$ for a homogeneous gas could imply that the gas is optically thin, which is not realistic for the nuclear environments considered in our sample. An optically thick cloud model with external heating of small dense clouds, i.e. with hot young stars blowing holes into the molecular structures, can make the $\mathrm{CO}(2-1)$ line stronger than the $\mathrm{CO}(1-0)$ line, if the temperature decreases by about 30-60 K (factor 2) from the $\tau=1$ surface for the $\mathrm{CO}(2-1)$ line to the $\tau=1$ surface for the CO (1-0) line (Braine \& Combes 1992). The higher opacity of the $\mathrm{CO}(2-1)$ line as compared to the $\mathrm{CO}(1-0)$ line, in almost all scenarios, leads to a higher beam filling factor for the $\mathrm{CO}(2-1)$ line and thus a line intensity ratio larger than unity.

Early evaluation of the $I_{\mathrm{CO}}$ to hydrogen mass $M\left(\mathrm{H}_{2}\right)$ conversion factor in galaxies by Maloney \& Black (1988) assumed thermalized CO rotational levels with $T_{\mathrm{ex}}=T_{\mathrm{kin}}$. This would predict $\mathrm{CO}(2-1) / \mathrm{CO}(1-0)$ brightness temperature ratios in the range of 0.9 to 1.1 in the Galaxy. In order to explain similar extragalactic data, Radford et al. (1991) show that sub-thermal excitation $\left(T_{\text {ex }}<T_{\text {kin }}\right)$ in the $n\left(\mathrm{H}_{2}\right)=10^{2}$ to $10^{3} \mathrm{~cm}^{-3}$ range could result in integrated $\mathrm{CO}(2-1) / \mathrm{CO}(1-0)$ line ratios in the 1.4-2 range, and beam-corrected (intrinsic) $T_{\mathrm{b}}(2-1) / T_{\mathrm{b}}(1-0)$ ratios in the range of $0.6-0.75$.

An evaluation of the $\mathrm{CO}$ properties requires consideration of the relative filling factor of the two CO transitions (Sect. 4). Here the conversion from the observed $T_{\mathrm{mb}}$ ratio to an intrinsic $T_{\mathrm{b}}$ ratio involves an antenna beam correction factor $\left(d_{\mathrm{s}}^{2}+\right.$ $\left.b_{21}^{2}\right) /\left(d_{\mathrm{s}}^{2}+b_{10}^{2}\right)$, which expresses the relative size of the source $d_{\mathrm{s}}$ and the beam size $b$ at the two transitions. For a representative size of $2 \mathrm{kpc}$ for the prominent $\mathrm{CO}$ emission regions in luminous FIR galaxies, the intrinsic $T_{\mathrm{b}}$ ratios will be reduced by a factor of $1.15-2.46$ (for velocities of 750 to $7500 \mathrm{~km} \mathrm{~s}^{-1}$ ) relative to the corresponding $T_{\mathrm{mb}}$ ratios. This range of values suggests that most of the observed ratios could have intrinsic $T_{\mathrm{b}}$ ratios around unity, which is consistent with a sub-thermal excitation of the low-density molecular medium (Radford et al. 1991). Our most extreme value of 3.0 for the OH MM source IR $15107+0724$ at $46 \mathrm{Mpc}$ would give a brightness temperature ratio of 1.4 .

The conversion of integrated $\mathrm{CO}(1-0)$ lines to $\mathrm{H}_{2}$ column densities is expressed as $N\left(\mathrm{H}_{2}\right) / I_{\mathrm{CO}(1-0)}=\alpha$ (Strong et al. 1988). These authors suggested a value $\alpha=2.3 \times$ $10^{20}$ molecules $\mathrm{cm}^{-2} /\left(\mathrm{K} \mathrm{km} \mathrm{s}^{-1}\right)$, using the properties of selfgravitating virialized molecular clouds of high optical depths. However, this value is not uniformly applicable for the Galaxy and for extragalactic nuclei. Studies of the Galactic Center using COBE data (Sodroski et al. 1995) and $\mathrm{C}^{18} \mathrm{O}$ and the mm dust emission (Dahmen et al. 1998) find that the conversion factor is a highly variable value, resulting in an overestimate of the mass in the nuclear region and an underestimate in the outer disk. 
Studies of the central regions of the nearby galaxies NGC 253 (Mauersberger et al. 1996b) and NGC 4945 (Mauersberger et al. 1996a) show an overestimate by a factor of 6-10. Studies of ULIRGs show that most of the CO luminosity comes from relatively low-density gas that is dynamically significant and is located in a molecular disk (Solomon et al. 1997; Downes \& Solomon 1998). A conservative estimate of the CO-to- $\mathrm{H}_{2}$ conversion $M\left(\mathrm{H}_{2}\right) / L_{\mathrm{CO}}^{\prime}=0.8 M_{\odot} /\left(\mathrm{K} \mathrm{km} \mathrm{s}^{-1} \mathrm{pc}^{2}\right)$ for such extragalactic nuclei is based on an $\alpha$ factor about 5 times lower than the value for self-gravitating clouds. This conversion ratio may be used to convert the $\mathrm{CO}(1-0)$ values listed in Table B.1 into $\mathrm{H}_{2}$ masses.

\section{High-density tracer molecules}

\subsection{Line luminosities}

The diagrams of line luminosity against the infrared luminosity for all molecular transitions considered (Figs. 1-3) show a wellbehaved distribution with a considerable width. The spread of the data points may be caused by a variety of effects, such as non-radiative heating of the gas, differences in the initial abundances, and the evolutionary changes of the ISM resulting from a starburst and the depletion of the high-density material (see Sect. 7 below). Data points at low luminosity that lie well below the center of the line luminosity distribution may result from using only the central pointing for nearby galaxies.

The HCN, HNC and $\mathrm{HCO}^{+}$molecules - The line luminosities of the source sample of the prominent tracer molecules are presented against the FIR luminosity in Fig. 2. The slopes for these three molecules are close to unity such that HCN (84 sources) has $s=0.98 \pm 0.06(r=0.89)$, HNC (28 sources) has $s=1.18 \pm 0.13(r=0.88)$, and $\mathrm{HCO}^{+}(42$ sources $)$ has $s=1.10 \pm 0.06(r=0.95)$. The tracer luminosities provide a slightly tighter relation with $L_{\mathrm{FIR}}$ than the $\mathrm{CO}(1-0)$ data as displayed in Fig. 1. Fits with slope $0.9 \pm 0.1$ for $\mathrm{HCN}$ versus $\mathrm{HCO}^{+}$ and with slope $1.4 \pm 0.4$ for $\mathrm{HCN}$ versus $\mathrm{CO}(1-0)$ have been noted by Nguyen-Q-Rieu et al. (1992). A slope of $0.97 \pm 0.05$ was found for HCN versus $L_{\text {FIR }}$ (Gao \& Solomon 2004a) and slopes of $0.88 \pm 0.15$ for $\mathrm{HNC}$ versus both $\mathrm{HCN}$ and $\mathrm{HCO}^{+}$ (Hüttemeister et al. 1995). The scatter of the distributions is about one decade in the main body of data points.

The CN molecule - The mm-wave spectra of the cyano radical $\mathrm{CN}$ have more complex characteristics than those of the other observed molecular species. The $\mathrm{CN} N=1-0$ line observations at Pico Veleta have been taken at the strongest feature, the $J=3 / 2-1 / 2$ transition at $113.491 \mathrm{GHz}$ that contains one third of the total intensity in the optically thin limit under Local Thermodynamic Equilibrium (LTE) conditions. For the SEST data for NGC 1068 , besides the $J=3 / 2-1 / 2$ feature a second $J=1 / 2-1 / 2$ feature is seen at $113.191 \mathrm{GHz}$ at a relative strength of 0.45 (Figs. A.2). This relative strength is close to the LTE value, which could suggest that the lines are optically thin (see Wang et al. 2004). The $\mathrm{CN} N=2-1$ transition data focus on the $J=5 / 2-3 / 2$ line at $226.659 \mathrm{GHz}$ that represents one sixth of the total intensity ( $\tau<1$; LTE). The CN data has been augmented with data for both transitions from Aalto et al. (2002).

Most of the $\mathrm{CN}$ detections are single broad features centered at the systemic velocity of the galaxies (Figs. A.1-A.3). Only Arp 220 and IR 05414 show double $N=1-0$ and $N=2-1$ $\mathrm{CN}$ components that cannot be attributed to other spingroups but only to the two nuclei of their respective systems.

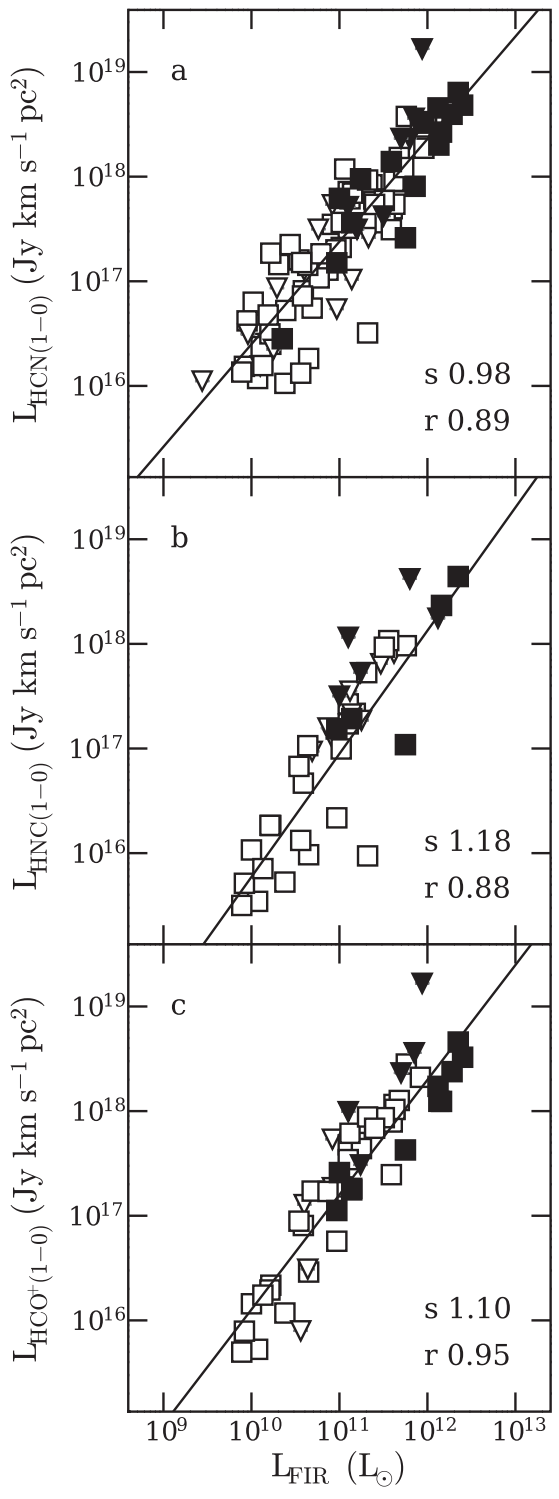

Fig. 2. a) Integrated $\mathrm{HCN}(1-0)$, b) integrated $\mathrm{HNC}(1-0)$, and c) integrated $\mathrm{HCO}^{+}(1-0)$ line luminosity versus FIR luminosity for the total sample. There are 84 reliable data points for HCN, 27 for HNC, and 42 for $\mathrm{HCO}^{+}$. The slope of the fitted line $(s)$ and the correlation coefficient $(r)$ are shown in the lower right corner of each frame. The displayed upper limits have not been used in the fits. The symbols are explained with Fig. 1.

The CN line luminosities in Fig. 3a,b show a distribution that is similar to that of other tracer molecules. A slope close to unity is found for $\mathrm{CN}(1-0)$ (19 sources): $s=1.03 \pm 0.13(r=0.89)$ and for $\mathrm{CN}(2-1)$ (13 sources): $s=1.14 \pm 0.17(r=0.89)$. The scatter of the distributions is less than one order of magnitude.

The CS molecule - Our CS (3-2) data from the PV 30$\mathrm{m}$ and SEST telescopes (Figs. A.1-A.3) have been augmented with detections from Mauersberger et al. (1989), Mauersberger \& Henkel (1989), and Wang et al. (2004). An early assessment of the CS emission in nearby galaxies shows a clear relation with $L_{\mathrm{CO}}$ and $L_{\mathrm{FIR}}$ and the star formation rate (Mauersberger \& Henkel 1989). The CS line luminosity (17 sources) versus the FIR luminosity has a higher slope, $s=1.56 \pm 0.14(r=0.95)$, as compared with a slope of unity for the much smaller sample of Mauersberger et al. (1989). This larger slope of CS as compared 


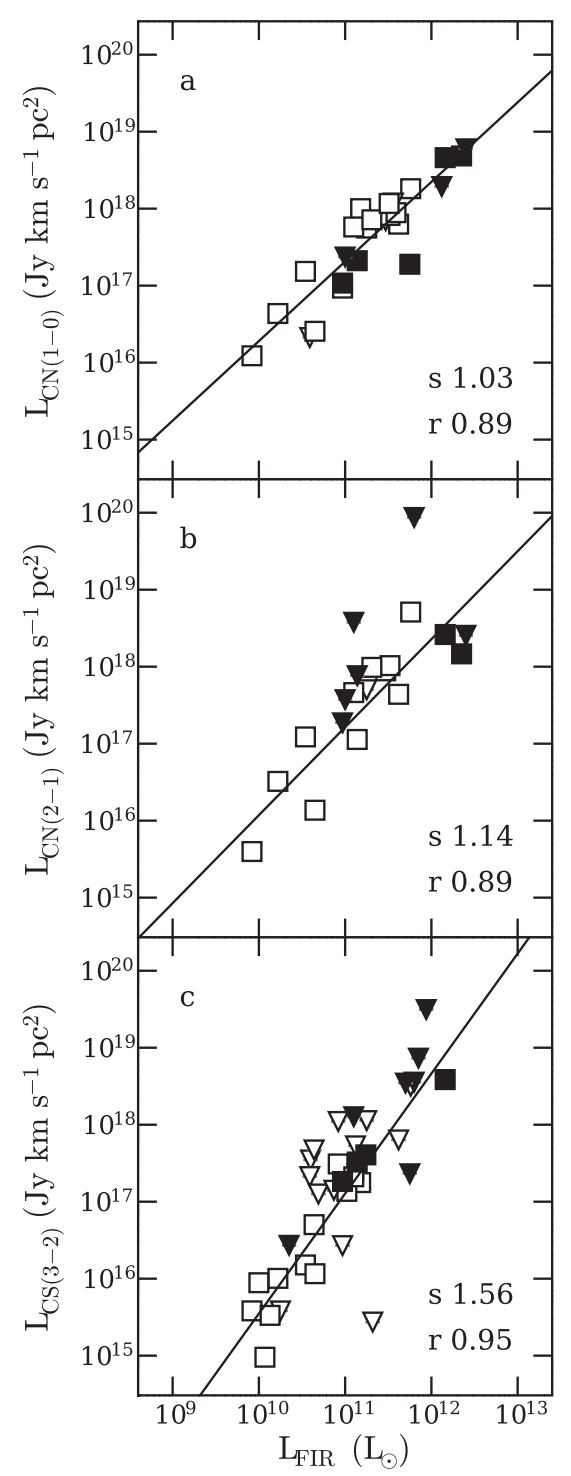

Fig. 3. a) Integrated $\mathrm{CN} N=(1-0)$, b) integrated $\mathrm{CN} N=(2-1)$, and c) integrated CS (3-2) line luminosity versus FIR luminosity for the total sample. There are 19 reliable data points for $\mathrm{CN}(1-0), 13$ for $\mathrm{CN}(2-1)$, and 17 for $\operatorname{CS}(3-2)$. The slope of the fitted curves $(s)$ and the correlation coefficient $(r)$ are shown in the lower right corner of each frame. No upper limits have been used in the fits. The symbols are described with Fig. 1.

to the other molecules under consideration indicates a stronger environmental dependence (Fig. 3c).

\subsection{Evaluating the relations}

The well-known Kennicutt-Schmidt laws relate to the surfacedensity of star formation $\left(\Sigma_{\mathrm{SFR}}\right)$ and the surface-density of the gas $\left(\Sigma_{\text {gas }}\right)$. On the basis of $\mathrm{HI}, \mathrm{CO}$, and $\mathrm{H} \alpha$ data for a large sample of spiral and starburst galaxies, Kennicutt (1998) finds that the $\Sigma_{\mathrm{SFR}}-\Sigma_{\text {gas }}$ relation has an exponent $\alpha=1.4\left(\Sigma_{\mathrm{SFR}} \propto \Sigma_{\text {gas }}^{\alpha}\right)$. Analogously the relation of the integrated luminosity of the high-density gas component involved in the star formation process and the integrated star formation rate (SFR) expressed as $L_{\text {FIR }}$ could also behave as a Kennicutt-Schmidt law. Krumholz \& Thompson (2007) made model predictions for the KennicuttSchmidt laws assuming a constant star formation efficiency.
They find that the line luminosity for molecules with a critical density lower than the average density of the system rises linearly with density. For lines with critical densities larger than the median gas density, the luminosity per unit volume increases faster than linearly with the density. The star formation rate also rises super-linearly with the gas density, and the combination of these two effects produces a close to linear correlation $(\alpha \sim 1)$ for the high critical density molecules and a superlinear relation for the low density tracers $(\alpha \sim 1.5$; the classic Kennicutt-Schmidt law). They also predict that generally there may be more intrinsic scatter in this relation for low critical density molecules than for high density tracers.

Our data mostly confirm the findings of Krumholz \& Thompson (2007). Our CO distributions (Fig. 1) show indices of $\alpha=1.40$ and 1.23 (the inverse of the slope $s$ in our diagrams) and have a relatively large spread. The relations between $L_{\mathrm{FIR}}$ and the line luminosities of high density tracers $\mathrm{HCN}(1-0)$, $\mathrm{HNC}(1-0), \mathrm{HCO}^{+}(1-0), \mathrm{CN}(1-0)$, and $\mathrm{CN}(2-1)$ are all linear within the errors (see Figs. 2 and 3). However, the relation for CS (3-2) is sub-linear with $\alpha=0.66$. Since CS has a critical density close to that of $\mathrm{HCN}$ and higher than that for $\mathrm{CO}$, CS is expected to have a linear relation (Krumholz \& Thompson 2007). This may indicate that this species is influenced by additional chemical and environmental effects.

\subsection{Tracer intensities relative to $C O(1-0)$}

In Figs. 4 and 5 the integrated intensities for the total sample are compared with the $\mathrm{CO}(1-0)$ emission that represents the larger scale low-density component. Ignoring NGC 7469, which seems to have unusual high ratios, and upper limits, the emission line ratio varies from 0.01 to 0.18 for $\mathrm{HCN}$, from 0.01 to 0.08 for $\mathrm{HNC}$, from 0.01 to 0.21 for $\mathrm{HCO}^{+}$, from 0.01 to 0.15 for $\mathrm{CN}$, and from 0.01 to 0.11 for CS. An early study of CS suggests a relative line ratio in the $0.01-0.08$ range (Mauersberger \& Henkel 1989). The upper range for the HCN/CO intensity ratio of our current sample is comparable with the sample of Gao \& Solomon (2004a). The range for $\mathrm{HNC}$ and $\mathrm{HCO}^{+}$is found to be (slightly) smaller than for $\mathrm{HCN}$ (when ignoring the outliers/upper limits) (see also Graciá-Carpio et al. 2006).

We note that the distribution of characteristic line ratios for all molecules increases with FIR luminosity, which gives an upwardly curved lower boundary for the distribution at higher $L_{\mathrm{FIR}}$. The highest values for all ratios are found at $L_{\mathrm{FIR}} \geq 10^{10.5} L_{\odot}$. As discussed in Sect. 7 below, a decreasing line strength of a high-density tracer as compared with $\mathrm{CO}$ during the course of a nuclear starburst would be the signature of the depletion of the nuclear high-density component.

\section{Modelling of a ULIRG outburst}

\subsection{The outburst model}

The FIR light curve of an ULIRG is determined by the radiative energy generated by a (circum-)nuclear starburst possible in hybrid mode with an AGN, that is re-radiated by a torus-like dusty environment. During such a FIR outburst, the low-density and high-density molecular components of the nuclear region evolve differently. The large-scale signature of the low-density gas, as represented by $\mathrm{CO}(1-0)$ emission, would only be partially altered by the centralized star formation process. On the other hand, the centrally concentrated high-density gas forms the structural basis for the star formation process and will be slowly consumed, destroyed or dispersed by the newly formed stars. 


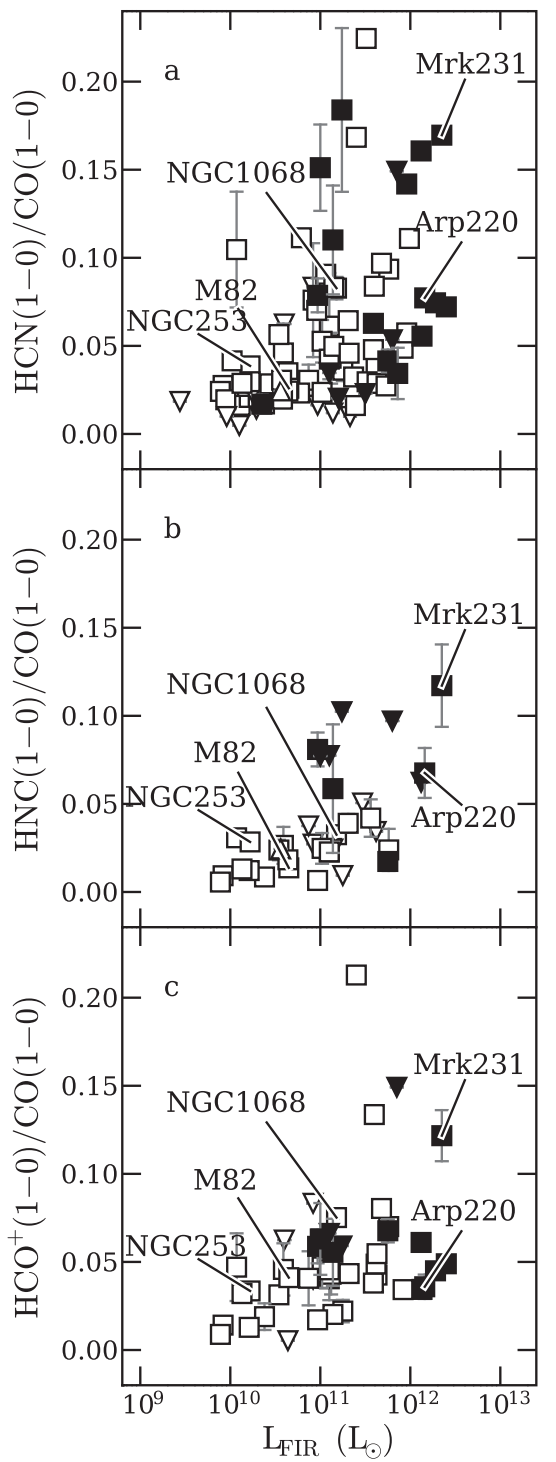

Fig. 4. The fraction of the high-density component relative to the lowdensity component. a) Integrated $\mathrm{HCN}(1-0) / \mathrm{CO}(1-0)$, b) integrated $\mathrm{HNC}(1-0) / \mathrm{CO}(1-0)$, and $\mathbf{c})$ integrated $\mathrm{HCO}^{+}(1-0) / \mathrm{CO}(1-0)$ line ratios as a function of FIR luminosity. The symbols are described with Fig. 1.

In a simplified scenario, the instantaneous FIR luminosity of the ULIRG represents the rate at which the nuclear activity generates FIR energy and consumes/destroys its high-density molecular material. In the absence of a representative FIR light curve with different timescales for the rise and fall of the FIR luminosity, we choose a diffusion-like expression as a response to a sudden start of the star formation. This function may be defined for $t>0$ as:

$L_{\mathrm{FIR}}(t)=1.35 L_{\mathrm{FIR}}(\max )\left(\frac{T}{t}\right)^{2.5} \mathrm{e}^{-T / t}$,

where $L_{\mathrm{FIR}}(\max )$ is the maximum luminosity and $T$ is the characteristic timescale of the burst. The duration of the burst (down to $1 \%$ of the peak) is on the order of $10 \times T$. A diffusion curve may not be the most appropriate representation of a FIR light curve but it resembles those obtained with starburst-driven models of the FIR evolution and color properties of ULIRGs (Loenen et al. 2006).

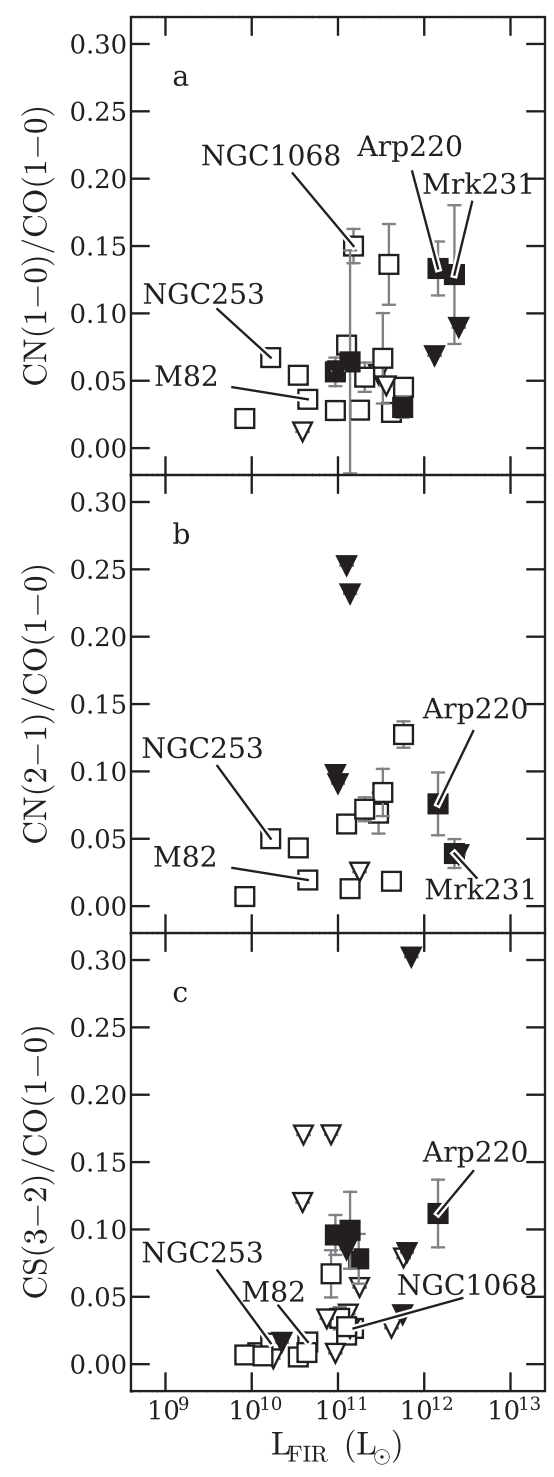

Fig. 5. The fraction of the high-density component relative to the lowdensity component. a) Integrated $\mathrm{CN}(1-0) / \mathrm{CO}(1-0)$, b) integrated $\mathrm{CN}(2-1) / \mathrm{CO}(1-0)$, and c) integrated $\mathrm{CS}(3-2) / \mathrm{CO}(1-0)$ line ratios versus FIR luminosity. The symbols are described with Fig. 1.

The initial line strength of the high-density $H D(t=0)$ component can be defined as a fraction $\beta_{0}$ of that of $L D$, the largerscale low-density component, that is assumed to remain unchanged during the outburst. As a result, the line ratio of the high- and low-density components varies with time during the FIR outburst as:

$\frac{H D(t)}{L D}=\beta_{0}\left[1-\Gamma \frac{\int_{0}^{t} L_{\mathrm{FIR}}(t) \mathrm{d} t}{E_{\mathrm{FIR}, \text { int }}}\right]$,

where $\Gamma$ is the total fraction of the initial $\beta_{0}$ of the $H D$ emission component that is destroyed (disappears) during the whole course of the outburst. The instantaneous $H D$ component depends on the fraction of the FIR energy generated up till time $t$ and the total FIR energy radiated during the outburst $E_{\mathrm{FIR}, \text { int }}$. 


\subsection{Modelling results}

The results obtained in these simulations are presented in Fig. 6 for three FIR light curves with peak luminosities log $L_{\mathrm{FIR}}(\max )=11,12$, and 12.5 in order to cover the range of observed FIR luminosities. The FIR light curves (upper frame) are used to determine the evolving luminosity of a highdensity component (middle frame), and the evolving emission ratio of the high- and low-density components (bottom frame). A light curve having a more delayed peak would give more rounded curves and the turn-around point would occur at higher $H D(t) / L D$ values.

This simple time-dependent scenario already shows some distinctive behavior of the high-density component during a FIR outburst, that is relevant to the high-density tracer data presented in this paper:

1) The rapid rise segment (indicated as (a) in Fig. 6) of the star formation process and the resulting FIR outburst ensure that the data points show rapid evolution and are relatively sparse at the onset of the curves. During the peak of the curve the depletion rate of the high-density component is highest. After the luminosity peak the data points in the decay segment of the three curves (indicated as (b) in Fig. 6) will be closer together. Because of the increased probability of finding a source in this state, our source samples will be dominated by sources in the intermediate and later stages of evolution.

2) The luminosity of the high-density component versus $L_{\mathrm{FIR}}$ will display a separation between the upward (a) and downward (b) luminosity segment due to the ongoing depletion (middle frame Fig. 6). Sources with the highest peak luminosities and the largest depletion factors follow the outermost evolutionary track and will end on the lower boundary of the distribution. This effect could add significantly to the scatter in the diagrams of the high density tracers versus $L_{\mathrm{FIR}}$.

3) The high- to low-density emission ratio versus FIR (bottom frame Fig. 6) reflects the phases of the FIR light curve: a rapid horizontal track for the rapid rise segment $(a)$ of the FIR luminosity with few data points, followed by a downward curved track with accumulation of data points at the lower boundary for the decay segments (b). After finishing the FIR light curve, a third buildup phase may take place with a steady re-building of the high-density molecular component in the central regions. A molecular buildup would prepare the galaxy for the next star formation outburst.

\subsection{Comparison with observations}

The observed relations between molecular line luminosities and FIR luminosities in Figs. 1-3 are in agreement with the simulations in the middle frame of Fig. 6. The observed scatter in the diagrams would partly result from differences in the rise and decay stages of an outburst. Furthermore, the track of the highest luminosity sources and $\mathrm{OH}$ MM would lie at the outer edges of the distribution. Similarly, the distribution of data points in an $L_{H D}-L_{\text {FIR }}$ diagram could display a steepening towards higher $L_{\text {FIR }}$ values (see Fig. 2).

The observed intensity ratios of tracer molecules relative to $\mathrm{CO}(1-0)$ show organized distributions (Figs. 4 and 5), that are in agreement with the simulations in Fig. 6c. The upper regions of the distributions are indeed sparsely populated as they represent (near-)initial values for sources. From the diagrams it follows that the initial fraction $\beta_{0}$ of the high-density component would be in the $0.08-0.18$ range, and the depletion rate $\Gamma$ for our sample of sources would lie in the $0.6-0.9$ range. Further
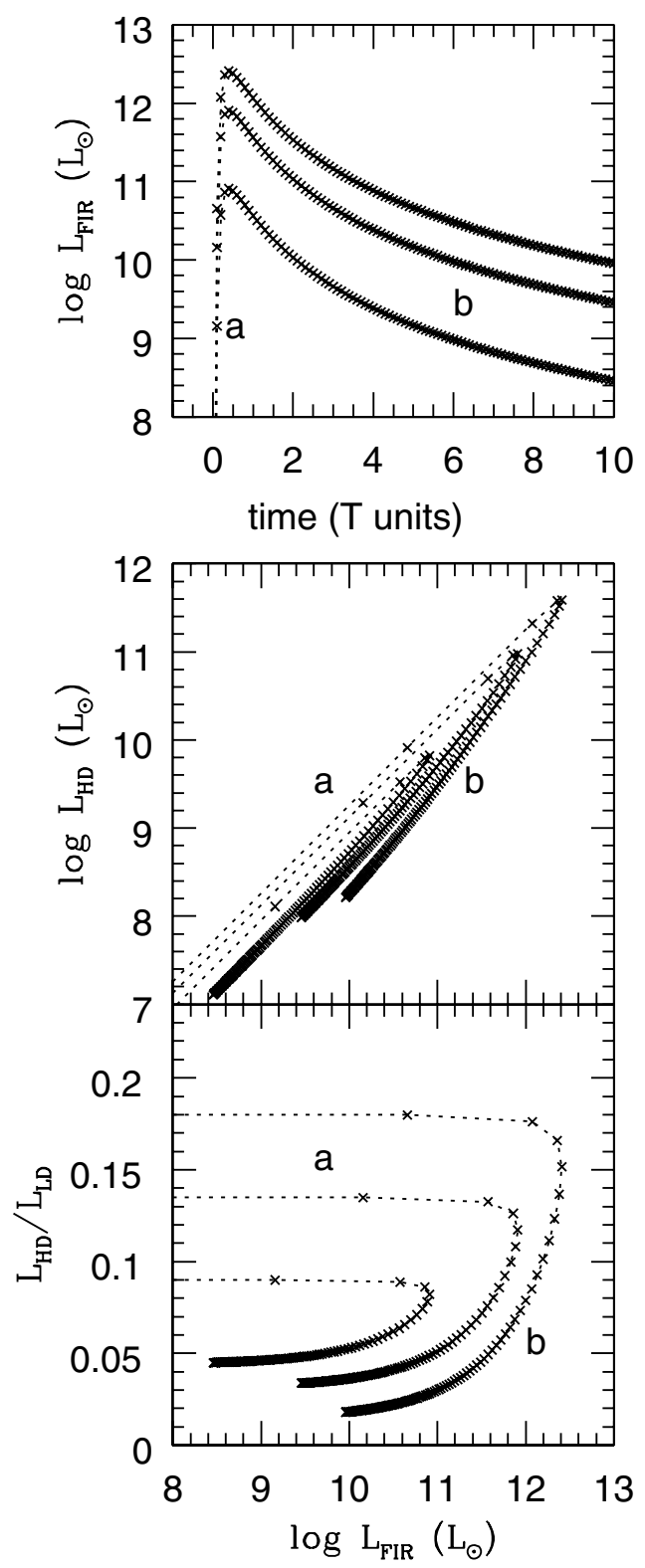

Fig. 6. Simulations of characteristic behavior of the high-density component during a FIR outburst. Labels (a) and (b) indicate the rapidly rising and the slower decaying segments of the outburst, respectively. Upper diagram: three $L_{\mathrm{FIR}}$ light curves versus time on a log-linear scale. The time is in units of $T$ as used in Eq. (1). Middle diagram: the associated luminosity of the high-density $(H D)$ component $L_{\mathrm{HD}}$ for the three light curves on a log-log scale. The parameters of the curves as described in Sect. 7 are: 1) the outer curve with initial $\beta_{0}=0.18, \log$ $L_{\mathrm{FIR}}(\max )=12.5$, and a depletion rate of $\Gamma=0.9$ during the course of the outburst; 2) the middle curve $0.135,12.0$, and 0.5 ; and 3 ) the inner curve $0.09,11.0$, and 0.4 . Lower diagram: the time-evolution of the ratio of high- and low-density (LD) components for the three FIR light curves of the above frames.

evolution of the outburst during the decay phase would result in the characteristically curved lower boundary observed in the diagrams. The highest FIR luminosity sources that have passed beyond their peak are also found at this lower boundary.

The distribution of $\mathrm{OH} \mathrm{MM}$ (and $\mathrm{H}_{2} \mathrm{CO} \mathrm{MM}$ ) are quite conspicuous in the diagrams. Some occupy the sparse region of rapid evolution at the highest $H D / L D$ intensity ratios (high $\beta_{0}$ ), 
while others have already evolved to the curved lower edge of the distribution, as seen clearly for the $\mathrm{HCN} / \mathrm{CO}$ ratio in Fig. 4 a.

\section{Molecular excitation environments}

The emission properties of Galactic and also extragalactic emission regions have been studied and interpreted by using two distinct excitation regimes: X-ray Dominated Regions (XDRs) and Photon Dominated Regions (PDRs) with far-ultraviolet radiation fields. These environments are quite distinguishable using the emission characteristics of high-density tracers in the cloud core regions and low-density tracers at the cloud surfaces (Lepp \& Dalgarno 1996; Meijerink \& Spaans 2005; Boger \& Sternberg 2005). The abundance and emission characteristics of the molecular and atomic lines depend strongly on the available (attainable) column density submitted to the radiation field and the strength of the radiation field.

Global environmental conditions - The PDR and XDR modelling of Meijerink \& Spaans (2005) and Meijerink et al. $(2006,2007)$ incorporate the physics and the chemistry in the local environment using many molecules and chemical reactions under local thermal balance. A large grid of density and radiative conditions was used to predict the cumulative column densities taking into account the radiation transfer in a representative slab-model incorporating XDR and PDR environments. This grid predicts column densities in all relevant energy states for the dominant molecules. All high-density tracer emissions are predominantly produced by regions with high hydrogen column densities. Relying on the predicted column densities for such a gas component, we restrict ourselves to a first order analysis of the observed line ratios using the ratios of calculated column densities.

The characteristics of both the PDR and XDR models depend on the parameterization of the radiative energy deposition rate per hydrogen nucleus $P=F_{\mathrm{FUV}} / n_{\mathrm{H}}$ or $F_{\mathrm{X}} / n_{\mathrm{H}}$ and suggest the following regimes:

1) For PDRs with high values of $P \geq 5 \times 10^{-4} \mathrm{erg} \mathrm{cm} \mathrm{s}^{-1}$ the column density ratios vary for all column densities $N_{\mathrm{H}} \geq$ $10^{21.5} \mathrm{~cm}^{-2}$ as:

$$
N\left(\mathrm{HCO}^{+}\right) / N(\mathrm{HCN})<N(\mathrm{HNC}) / N(\mathrm{HCN}) \leq 1
$$

while for smaller values for $N_{\mathrm{H}}$, the ratios switch around such that:

$$
N(\mathrm{HNC}) / N(\mathrm{HCN}) \ll 1 \leq N\left(\mathrm{HCO}^{+}\right) / N(\mathrm{HCN})
$$

and for lower $P \approx 5 \times 10^{-6} \mathrm{erg} \mathrm{cm} \mathrm{s}^{-1}$ the column density ratios vary as:

$$
1 \leq N(\mathrm{HNC}) / N(\mathrm{HCN}) \approx N\left(\mathrm{HCO}^{+}\right) / N(\mathrm{HCN}) .
$$

2) For XDRs the radiation penetrates deeper into the cloud volume than for PDRs. For stronger XDR radiation fields $(P \geq$ $5 \times 10^{-4} \mathrm{erg} \mathrm{cm} \mathrm{s}^{-1}$ ) and for column densities above the transition point $N_{\mathrm{H}} \approx 10^{22.5} \mathrm{~cm}^{-2}$, the observable molecular column densities have ratios:

$$
1 \leq N(\mathrm{HNC}) / N(\mathrm{HCN}) \leq N\left(\mathrm{HCO}^{+}\right) / N(\mathrm{HCN}),
$$

and below this $N_{\mathrm{H}}$ transition point:

$$
N(\mathrm{HNC}) / N(\mathrm{HCN}) \ll N\left(\mathrm{HCO}^{+}\right) / N(\mathrm{HCN}) \leq 1,
$$

while for lower $P \approx 5 \times 10^{-6} \mathrm{erg} \mathrm{cm} \mathrm{s}^{-1}$ the column density ratios vary as:

$$
N\left(\mathrm{HCO}^{+}\right) / N(\mathrm{HCN}) \ll N(\mathrm{HNC}) / N(\mathrm{HCN}) \approx 1 .
$$

3) For PDR conditions the column density ratio $N(\mathrm{CN}) / N(\mathrm{HCN})$ varies from 0.5 to 2.0 over the respective density range of $10^{6}$ to $10^{4} \mathrm{~cm}^{-3}$.

4) For XDR conditions the column density ratio is $N(\mathrm{CN}) / N(\mathrm{HCN}) \gg 1$ and may become as large as 10 .

5) For PDR conditions at $N_{\mathrm{H}} \geq 10^{22} \mathrm{~cm}^{-2}$ the column density ratio $N(\mathrm{CS}) / N(\mathrm{HCN}) \geq 1$. At lower column densities the ratio may become $\leq 1$, which makes $\mathrm{CS}$ a column density indicator.

6) For XDR conditions for the whole column density range at low values of $P \approx 5 \times 10^{-6} \mathrm{erg} \mathrm{cm} \mathrm{s}^{-1}$ the column density ratio is $N(\mathrm{CS}) / N(\mathrm{HCN}) \geq 1$. At higher values of $P$ the ratio remains $\geq 1$ for $N_{\mathrm{H}} \geq 10^{23} \mathrm{~cm}^{-2}$ and is $\leq 1$ for lower $N_{\mathrm{H}}$, again making it column density sensitive.

Evolutionary environmental conditions - The characteristics described above are representative of a steady-state nuclear excitation environment. Realistically the behavior of molecules tracing the high-density component is affected by changing evolutionary and environmental effects that influence their abundance and the exciting radiation fields.

Assuming that FIR luminous sources are mostly powered by (circum-)nuclear starbursts, the evolving star formation will generate supernovae with shocks enhancing the cosmic ray flux, and produce new generations of X-ray binaries. Because these star formation products alter the excitation environment, an environment initially dominated by PDR conditions could change into one with a combination of PDR and XDR conditions during the later stages of the outburst (for M 82 see: García-Burillo et al. 2002; Spaans \& Meijerink 2007). This will modify the line ratios from their steady-state conditions discussed above. For instance, increased cosmic ray production in shocks would enhance the relative $\mathrm{HCO}^{+}$abundance while shocks also would decrease the relative HNC abundance (Wootten 1981; Elitzur 1983; Schilke et al. 1992). We will refer to this evolved starburst stage as being in a "late-stage" as compared with an "early-stage" starburst where these effects do not yet play a role.

During the late-stage starburst also the density and temperature structure of the ISM and its clumping would be affected, resulting in chemical changes such as the selective destruction of HNC in favor of HCN at higher temperatures (see Sect. 2). In addition, a certain fraction of the high-density medium will be used/destroyed by the star formation process. As a result the line emission may originate in regions of decreasing column densities, which modifies the observed line ratios.

\section{Diagnostics with tracer lines}

In the absence of multi-transition studies using LVG simulations involving higher $J$ transitions, we make the assumption that the integrated line ratios have a diagnostic value that is equivalent to the ratios of column densities for the observable molecular species. The global characteristics of molecular column densities described in the previous section for XDR- and PDR-dominance may thus be used for a first diagnosis of the excitation environments.

\subsection{High-density tracer ratios versus $L_{\mathrm{FIR}}$}

Diagrams of relative intensities for $\mathrm{HCN}, \mathrm{HNC}, \mathrm{HCO}^{+}, \mathrm{CN}$, and CS are presented in Figs. 7 and 8. Horizontal lines for a ratio 


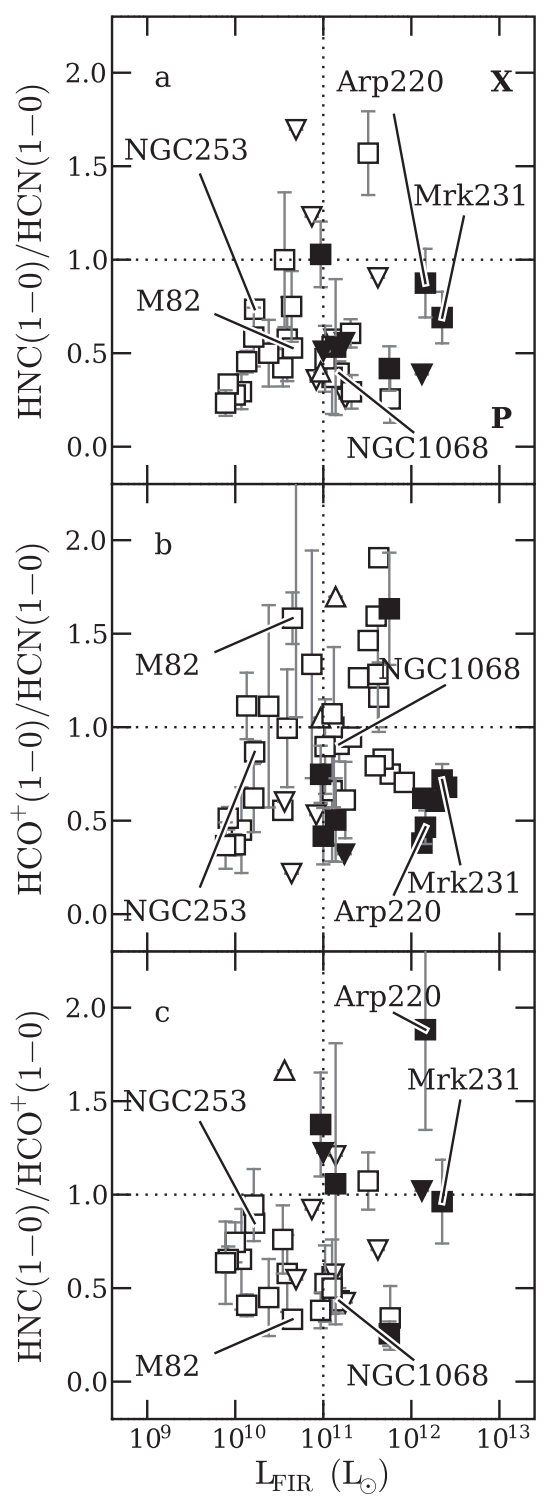

Fig. 7. High-density tracer line ratios versus FIR luminosity. a) The integrated $\mathrm{HNC}(1-0) / \mathrm{HCN}(1-0)$ line intensity ratio, b) the integrated $\mathrm{HCO}^{+}(1-0) / \mathrm{HCN}(1-0)$ line intensity ratio, and $\left.\mathbf{c}\right)$ the integrated $\mathrm{HNC}(1-0) / \mathrm{HCO}^{+}(1-0)$ line intensity ratio. The dotted lines mark the predicted range of XDR-PDR excitation using first-order modelling diagnostics. In the upper frame the PDRs lie below the line and the XDRs above. These labels have been omitted in the two lower frames (where $\mathrm{X}$ would be in the upper range of frame $\mathbf{b}$ ) and in the lower range of frame c)) because non-standard heating effects on $\mathrm{HCO}^{+}$and $\mathrm{HNC}$ (see Sect. 9.1) causes a shift of the data points and makes labelling nonappropriate. The symbols are described with Fig. 1.

equal to unity (and two for $\mathrm{CN}$ ) have been added to the diagrams in order to test a first order division between PDR and $\mathrm{XDR}$ line ratios as derived in the previous section. However, we find that the unity value is not a reliable boundary for ratios with $\mathrm{HCO}^{+}$. For $\mathrm{CS}$ this line separates high and low column densities. The line ratios are predominantly determined by regions having the highest molecular column densities (Meijerink et al. 2007). All diagrams show some systematic behavior in that the $\mathrm{OH}$ MM sources cluster together and that there could be a wedge-like upper boundary for the sources at high luminosities.
$\mathrm{HCN}, \mathrm{HNC}$ and $\mathrm{HCO}^{+}$lines - The observed ratios of $\mathrm{HCN}$, $\mathrm{HNC}$, and $\mathrm{HCO}^{+}$in Fig. 7 show a range of values between 0.2 and 2.0. Modelling results indicate that the $\mathrm{HCO}^{+} / \mathrm{HCN}$ ratio in most PDR environments is expected to be smaller than unity, while in $\mathrm{XDRs}$ the $\mathrm{HCO}^{+} / \mathrm{HCN}$ line ratio becomes $\geq 1$ for the higher column densities. The PDR sources are expected to lie in the lower parts $(\leq 1)$ of the upper two diagrams and in the upper part $(\geq 1)$ of the bottom diagram of Fig. 7 .

The observed HNC/HCN data shows significant clustering at 0.5 across the whole $L_{\text {FIR }}$ range, which could indicate that the $\mathrm{HNC} / \mathrm{HCN}$ ratios have been lowered by the evolving starburst excitation. In the $\mathrm{HCO}^{+} / \mathrm{HCN}$ diagram in $\mathrm{Fig}$. $7 \mathrm{~b}$, the $\mathrm{OH} \mathrm{MM}$ sources are well confined to the 0.3 and 0.8 range with only IC 694 (Arp299A, IR $11257+5850$ A) as the exception. Rather than finding a steady decrease of the observed $\mathrm{HCO}^{+} / \mathrm{HCN}$ with increasing $L_{\mathrm{FIR}}$ (Graciá-Carpio et al. 2006), our larger sample presents a more complicated picture that hints at (systematic) evolutionary influences on the nuclear medium.

The diagrams of Fig. 7 display systematic behavior related to the $\mathrm{HCO}^{+}$and $\mathrm{HNC}$ characteristics. The group of megamaser sources (mostly) lies to the right of the vertical line of $L_{\mathrm{FIR}}>$ $10^{11} L_{\odot}$ but they are in the PDR regions of all three diagrams. They are mostly high $N_{\mathrm{H}}$ and pure PDR sources. On the other hand, the group of sources left of that vertical line lies in the PDR regime for the $\mathrm{HNC} / \mathrm{HCN}$ ratio, straddles the XDR-PDR dividing line for the $\mathrm{HCO}^{+} / \mathrm{HCN}$ ratio, and lies in the XDR region for the $\mathrm{HNC} / \mathrm{HCO}^{+}$ratio. This effect can be attributed to $\mathrm{HCO}^{+}$ enhancement and HNC depletion during a late-stage starburst phase, that pushes PDR sources to a first-order XDR regime in the two bottom diagrams. This late-PDR behavior is exemplified by the late starburst M 82 .

The lowest values of $\mathrm{HNC} / \mathrm{HCO}^{+}$for high $L_{\mathrm{FIR}}$ sources are for OH MM IC 694 and the two AGN sources NGC 1068 and NGC 6240, which are all $\mathrm{H}_{2} \mathrm{O}$ emitters. NGC 6240 is not an $\mathrm{OH}$ MM but is similar to Arp 220 in its properties. Both have dominant (circum-)nuclear starburst activity and NGC 6240 also has a shock-heated molecular emission region located between its two AGN nuclei (Tacconi et al. 1999; Baan et al. 2007; Iono et al. 2007). The emission of the ring of starformation in NGC 1068 (Schinnerer et al. 2000) also falls within the SEST observing beam; this would dominate the signature of the source and results in different ratios than for IRAM 30 m data (Usero et al. 2004). As a result of these characteristics, the three sources lie in the PDR region of Fig. 7a and in the XDR region of Fig. 7c. Alternatively, the emission from these sources could be dominated by low $N_{\mathrm{H}}$ emission regions, where the theoretical line ratios are reversed compared to the high $N_{\mathrm{H}}$ cases (Sect. 8).

The evolved starburst behavior displayed in Fig. 7 shows that the first-order PDR-XDR division of the three diagrams may not be optimal. The most reliable tracer of PDR versus XDR behavior appears to be the $\mathrm{HNC} / \mathrm{HCN}$ ratio, because it is least affected by late-stage starburst conditions. Using this ratio would also imply that most sources of our sample are PDR dominated starburst sources.

CN and CS lines - The $N(\mathrm{CN}) / N(\mathrm{HCN})$ ratios would lie below 2.0 for PDRs and (far) above for XDRs (Meijerink et al. 2006). In Fig. 8ab any XDR sources would lie (far) above the line in the two diagrams. The $\mathrm{OH} M M$ have a typical $\mathrm{CN}(1-0) / \mathrm{HCN}$ ratio in the $0.4-0.8$ range, except for Arp 220 with a value of 1.7. The PDR-XDR division appears well situated in the diagram.

The $N(\mathrm{CS}) / N(\mathrm{HCN})$ ratio should be $\geq 1$ at high column densities for both XDRs and PDRs (Fig. 8c). The observed 


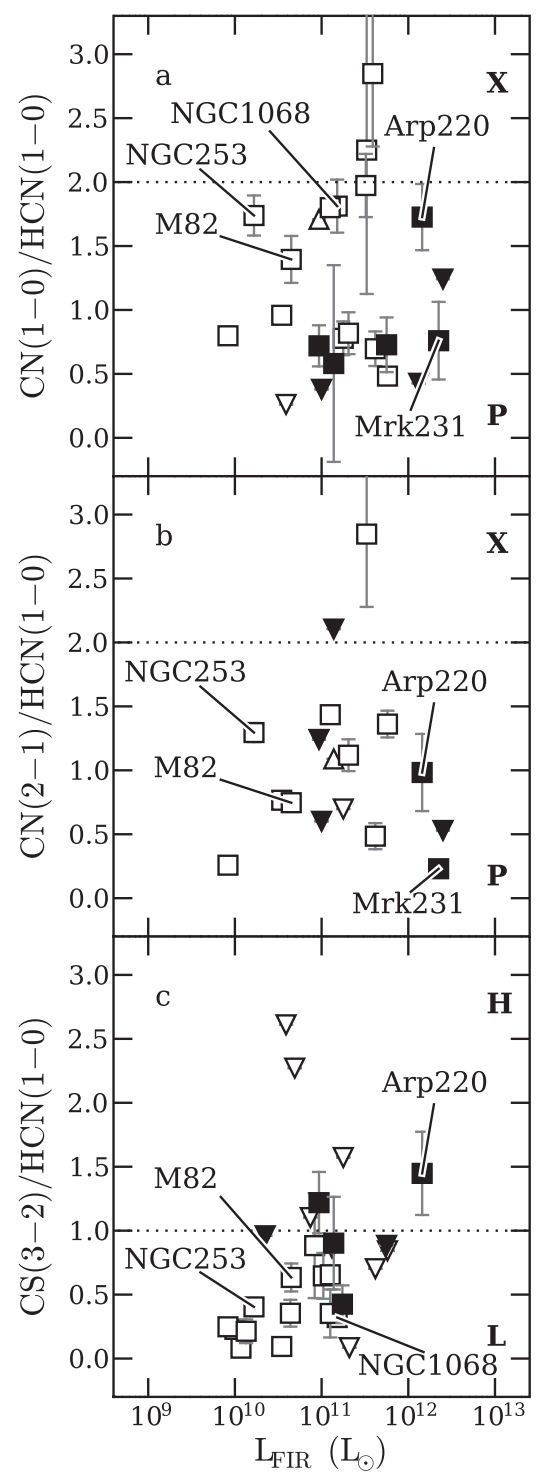

Fig. 8. $\mathrm{CN} / \mathrm{HCN}$ and $\mathrm{CS} / \mathrm{HCN}$ line ratios versus FIR luminosity. a) The integrated $\mathrm{CN}(1-0) / \mathrm{HCN}(1-0)$ line intensity ratio, b) the integrated $\mathrm{CN}(2-1) / \mathrm{HCN}(1-0)$ line intensity ratio, and c) the integrated $\mathrm{CS}(3-2) / \mathrm{HCN}(1-0)$ line intensity ratio. The symbols $\mathrm{X}$ and $\mathrm{P}$ and the dotted lines mark the range of values for XDRs and PDRs. For the $\mathrm{CS} / \mathrm{HCN}$ diagram the dotted line divides the range for low- and highcolumn densities (L \& H). The symbols are described with Fig. 1.

$\mathrm{CS}(3-2) / \mathrm{HCN}(1-0)$ ratio increases with increasing $L_{\mathrm{FIR}}$, which suggests a correlation with column density.

\subsection{High-density tracer ratios versus each other}

Tracers $\mathrm{HCN}, \mathrm{HCO}^{+}$, and $\mathrm{HNC}-$ The line ratios of highdensity tracer molecules $\mathrm{HCN}, \mathrm{HCO}^{+}$, and $\mathrm{HNC}$ may be compared to each other in order to discern collective behavior (Fig. 9). There is a significant spread in data points and a separation of a group of mostly $\mathrm{OH} \mathrm{MM}$ from an extended group of non-OH MM. The discrepant OH MM point is again IC 694.

The diagrams of Fig. 9 may be interpreted in the framework of dominant PDR and XDR characteristics of the emission regions using only the (more dependable) $\mathrm{HNC} / \mathrm{HCN}$ ratio (see Sect. 8). The data points have extended (slanted) distributions in all three frames with most sources lying on the PDR side of the three dividing lines.

The dependence on density is strongest for $\mathrm{HCO}^{+}($Meijerink et al. 2007). In the top-left frame, the diagonal structure represents a change from higher (bottom-right) to lower (top-left) densities with Arp 220 and other $\mathrm{OH}$ MM lying at the high density extreme. $\mathrm{HNC}$ depletion and $\mathrm{HCO}^{+}$enhancement due to evolved starburst environmental effects (see Sect. 8) may account for the bunching of data points, and the fact that some sources have moved close to the XDR-PDR boundary.

Tracers $\mathbf{C N}$ and $\mathbf{C S}-$ The $\mathrm{CN} / \mathrm{HNC}$ and $\mathrm{CN} / \mathrm{HCO}^{+}$versus $\mathrm{CN} / \mathrm{HCN}$ distributions show a significant spread with the $\mathrm{OH} \mathrm{MM}$ concentrated at lower $\mathrm{CN} / \mathrm{HNC}$ ratios (Fig. 10). The first order diagnostics discussed in Sect. 8 indicates that all sources have a $\mathrm{CN} / \mathrm{HCN}$ ratio close to unity, which is consistent with PDR environments. There is a hint of a gap in the source distribution at $\mathrm{CN} / \mathrm{HCN} \approx 1$. The slant in the data distribution in the bottom frame of Fig. 10 (as compared with the top frame) results from the $\mathrm{HCO}^{+}$enhancement for part of the sample.

The CS diagrams (Figs. 10c,d) display a clear extended structure with the $\mathrm{OH} \mathrm{MM}$ showing high ratios against $\mathrm{HNC}$, $\mathrm{HCO}^{+}$and $\mathrm{HCN}$. Since $\mathrm{CS}$ is to first order a column density tracer (see Sects. 2 and 8), this linear variation in the relative strength of CS is a consequence of seeing more of the highest density components with a high column density. ULIRGs/OH MM with high column densities have high ratios and moderate (low) power FIR sources like NGC 1808, M 83, and Maffei 2 have lower values.

Comparisons with $\mathrm{CO}$ - A diagnostic diagram may be constructed using the ratios of tracers with $\mathrm{CO}(1-0)$ (Fig. 11). These diagrams also display a significant (diagonal) spread in data points and a separation between $\mathrm{OH} \mathrm{MM}$ and the rest of the data points. The $\mathrm{HCO}^{+}$enhancement and the less apparent HNC depletion may account for the curved distributions of the bottom-left and top-right frames. As presented in previous sections, the ratios of high-density tracers with $\mathrm{CO}$ would be evolutionary indicators that reduce in value during the course of the outburst. Depending on their $L_{\mathrm{FIR}}(\max )$, the sources during early evolutionary stages have a starting point further towards the topright side of each frame, while more evolved sources or those with smaller $L_{\mathrm{FIR}}(\max )$ will appear more towards the bottomleft side. The relative locations of (late-stage) M 82 and (earlystage low $\left.L_{\mathrm{FIR}}(\max )\right)$ NGC 253, and ULIRGs/OH MM Arp 220 and Mrk 231 are consistent with this picture. While there is no one-to-one interpretation of relative positions of individual data points, the collective paths would support FIR-related evolution from higher $L_{\mathrm{FIR}}$ and PDR-dominated sources in early stages of evolution to XDR-like sources at lower $L_{\mathrm{FIR}}$ (see Figs. 9-12). Detailed modelling needs to confirm the evolutionary paths followed by the nuclear activity having different initial conditions.

\subsection{Diagnostics of the nuclear energy source}

The variation of the line intensity ratios has also been connected to the AGN or starburst nucleus (SBN) nature of the nuclear power sources (Kohno et al. 2001; Graciá-Carpio et al. 2006). Both authors suggest that the data points with $\mathrm{HCO}^{+} / \mathrm{HCN} \leq 0.55$ would indicate AGN activity and that higher values indicate a composite AGN nature.

The $(\log -\log )$ diagram of $\mathrm{HCO}^{+} / \mathrm{HCN}$ versus the evolutionary parameter of the $\mathrm{HCN} / \mathrm{CO}$ ratio (Fig. 12) shows a continuous distribution for our larger sample (see Sects. 6.3 and 7) that confirms the distribution of data points presented earlier (Kohno et al. 2001; Graciá-Carpio et al. 2006). The central ridge that 


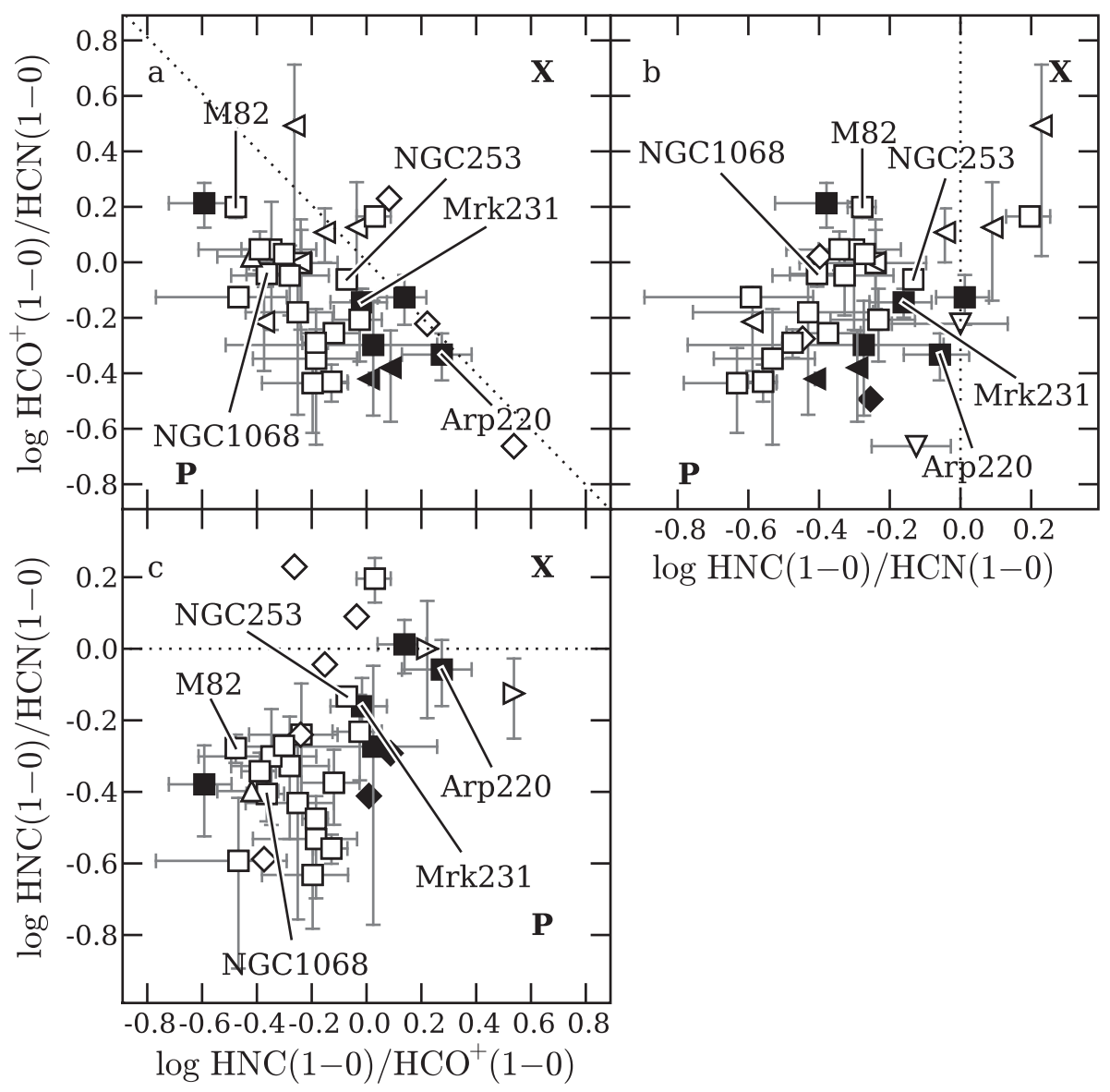

Fig. 9. The integrated line ratios of $\mathrm{HCN}(1-0)$, $\mathrm{HNC}(1-0)$, and $\mathrm{HCO}^{+}(1-0)$ versus each other. a) Integrated $\mathrm{HCO}^{+}(1-0) / \mathrm{HCN}(1-0)$ versus $\mathrm{HNC}(1-0) / \mathrm{HCO}^{+}(1-0)$ ratios; b) integrated $\mathrm{HCO}^{+}(1-0) / \mathrm{HCN}(1-0)$ versus $\mathrm{HNC}(1-0) / \mathrm{HCN}(1-0)$ ratios; and c) integrated $\mathrm{HNC}(1-0) / \mathrm{HCN}(1-0) \quad$ versus $\mathrm{HNC}(1-0) / \mathrm{HCO}^{+}(1-0)$ ratios. The $\mathrm{P}$ and $\mathrm{X}$ and the dotted lines mark the regions of the line ratios of predominantly photon-dominated regions (PDRs) and predominantly X-raydominated regions (XDRs) using only the $\mathrm{HNC} / \mathrm{HCN}$ ratio. The symbols are described with Fig. 1.
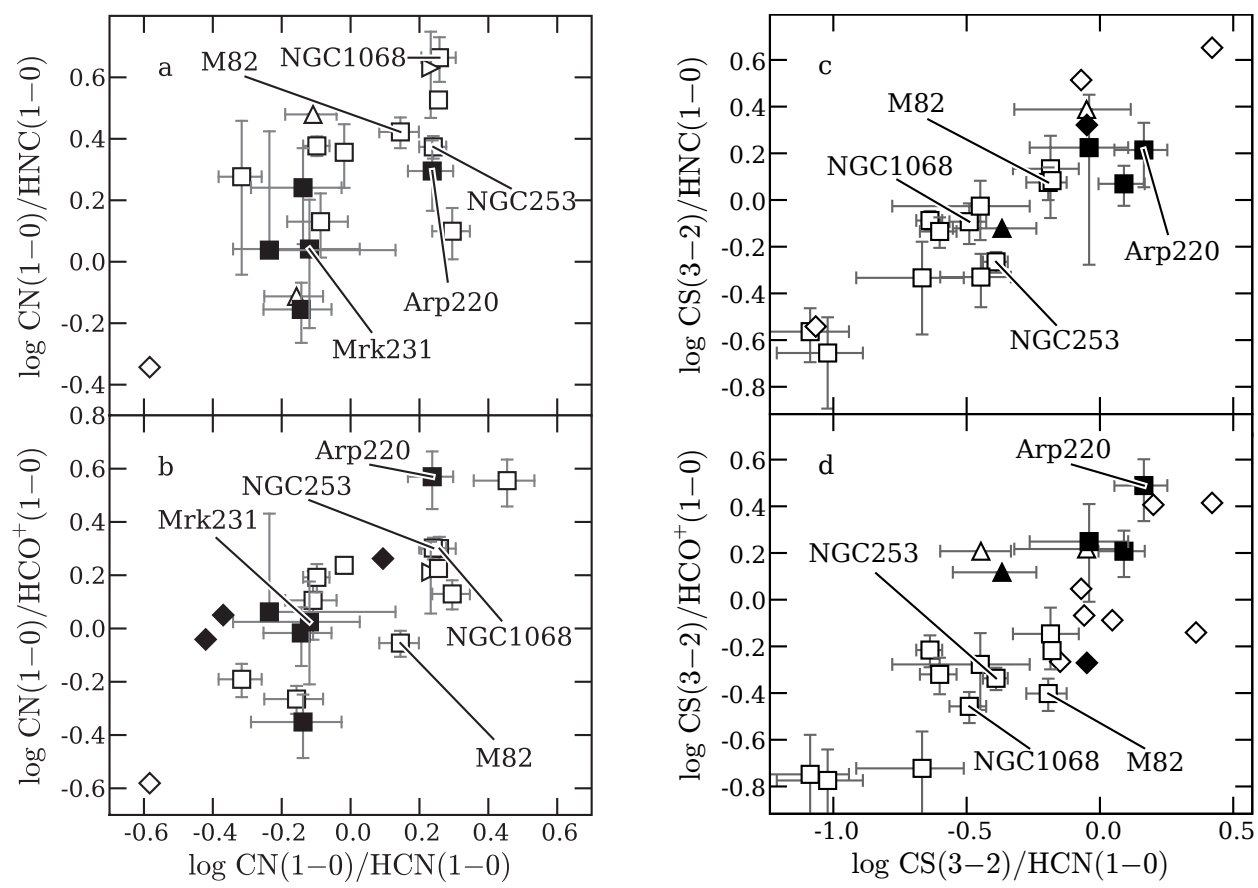

Fig. 10. The $\mathrm{CN}(1-0)$ and $\mathrm{CS}(3-2)$ lines compared with other high-density tracers. a) The $\mathrm{CN} / \mathrm{HCN}$ ratio versus the $\mathrm{CN} / \mathrm{HNC}$ ratio and b) versus the $\mathrm{CN} / \mathrm{HCO}^{+}$ratio. c) $\mathrm{The} \mathrm{CS} / \mathrm{HCN}$ ratio versus the $\mathrm{CS} / \mathrm{HNC}$ ratio and d) versus the $\mathrm{CS} / \mathrm{HCO}^{+}$ratio. The symbols are described with Fig. 1 .

accounts for most of the data points shows an inverse-linear relation between the two ratios, which suggests a general depletion of $\mathrm{HCN}$ relative to $\mathrm{CO}$ and $\mathrm{HCO}^{+}$during the course of the outburst. In our evolutionary scenario, a source moves from (bottom) right to (upper) left in the diagram during the decay segment of the nuclear outburst. M 82 represents a later stage of starburst evolution and is located at the upper left, since its late-PDR nature also enhances $\mathrm{HCO}^{+}$. $\mathrm{OH}$ MMs and ULIRGs may start at the right side of Fig. 12, while less dominant starbursts have a starting point more at the center or even on the 

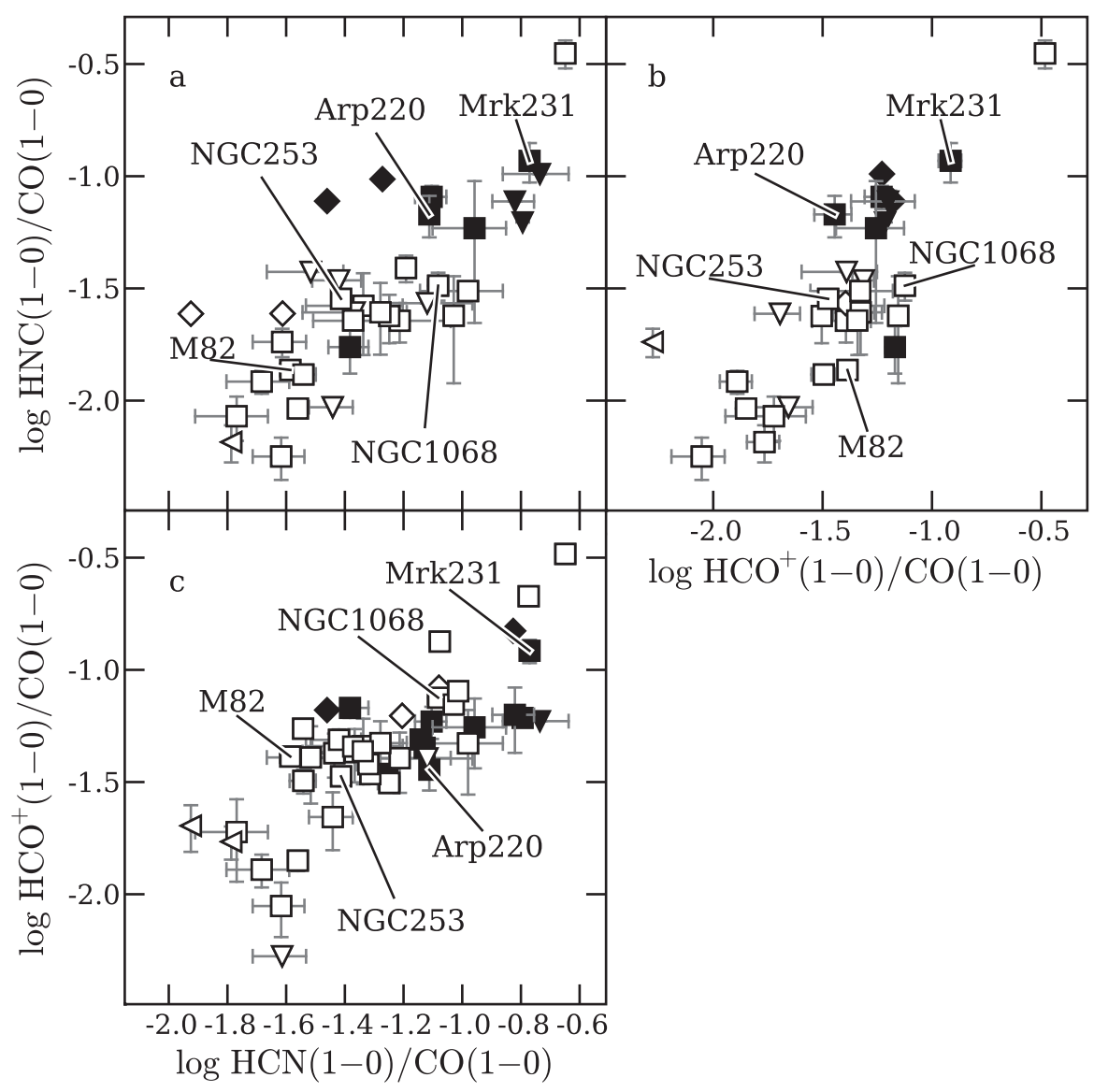

Fig. 11. Integrated line ratios of $\mathrm{HCN}(1-0)$, $\mathrm{HNC}(1-0)$, and $\mathrm{HCO}^{+}(1-0)$ versus $\mathrm{CO}(1-0)$. The filled squares are values for $\mathrm{OH}$ and $\mathrm{H}_{2} \mathrm{CO}$ megamaser galaxies, while the open squares are values for mostly nearby starburst galaxies. a) Integrated $\mathrm{HNC}(1-0) / \mathrm{CO}(1-0)$ versus $\mathrm{HCN}(1-0) / \mathrm{CO}(1-0)$ ratios; b) integrated $\mathrm{HNC}(1-0) / \mathrm{CO}(1-0) \quad$ versus $\mathrm{HCO}^{+}(1-0) / \mathrm{CO}(1-0)$ ratios; and c) integrated $\mathrm{HCO}^{+}(1-0) / \mathrm{CO}(1-0)$ versus $\mathrm{HCN}(1-0) / \mathrm{CO}(1-0)$ ratios. The symbols are described with Fig. 1.

left hand side of the diagram. NGC 253 is representing such a case.

First order diagnostics (Sect. 8) relates the $\mathrm{HCO}^{+} / \mathrm{HCN}$ ratio to PDR and XDR signatures rather than to the nature of central power source. We hereby assume that high $N_{\mathrm{H}}$ PDRs dominate the emission signatures and produce the low values for the $\mathrm{HCO}^{+} / \mathrm{HCN}$ ratio, rather than low $N_{\mathrm{H}}$ XDRs. Of the four $\mathrm{OH} \mathrm{MM}$ sources with the lowest $(\leq 0.55$ or $\log \leq$ -0.26) ratios (Arp 220, IRAS 11506-3851, IRAS 15107+0724, and Mrk 273), the radio classification only indicates a possible AGN in IRAS 15107+0724 (Baan \& Klöckner 2006). NIR classification suggests an AGN power contribution of $50 \%$ in Mrk 273 (Genzel et al. 1998). Both these classifications suggest a significant AGN presence in Mrk 231, which has a ratio here of 0.72 (higher than in e.g. Graciá-Carpio et al. 2006). Observed $\mathrm{HCO}^{+} / \mathrm{HCN}$ values are also higher than 0.55 for the starburst plus AGN source NGC 1068 (0.9; Usero et al. 2004) and the nearby starburst-dominated sources NGC $253(0.9)$ and M 82 (1.6; Fuente et al. 2005).

Therefore, the predicted properties of the nuclear ISM during the evolution of the starburst may well account for the data points in Fig. 12 and particularly the (inverse-linear) central ridge. If the observed trend were affected by the presence of an $\mathrm{AGN}$, its influence on the excitation environment of the nucleus would have to be further confirmed by more detailed modelling. The (circum-) nuclear starburst would easily outshine the compact AGN region and dominate the emission signature in low spatial resolution data.

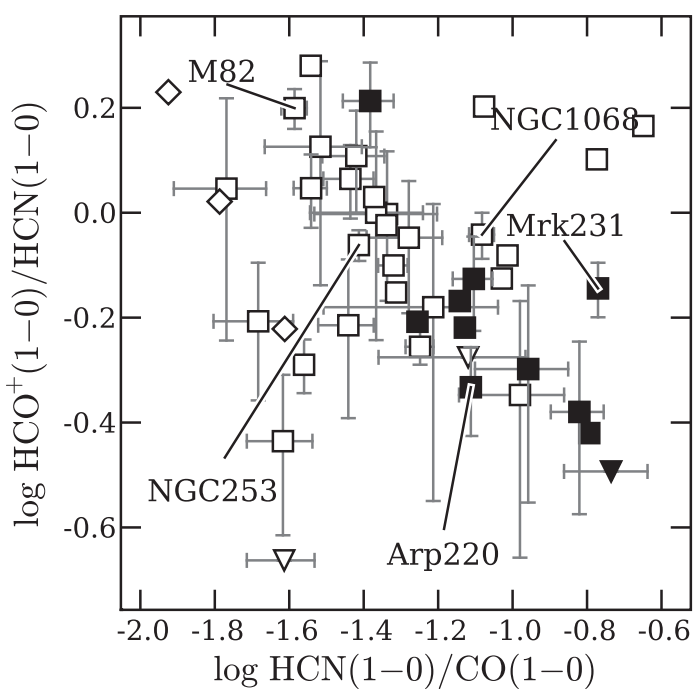

Fig. 12. The $\mathrm{HCO}^{+}(1-0) / \mathrm{HCN}(1-0)$ ratio versus the $\mathrm{HCN}(1-0) / \mathrm{CO}(1-0)$ ratio. The symbols are described with Fig. 1.

\section{Summary}

Molecular line emissions in FIR-luminous galaxies are tools for multi-dimensional diagnostics of the environmental parameters in the nuclear ISM and the heating processes resulting from the nuclear activity. 
A proper evaluation of integrated emission lines and their diagnostic line ratios can be achieved after synthesis and modelling of a representative description of the integrated nuclear molecular medium under a wide variety of AGN- and starburstrelated circumstances. The molecular information in this paper has been diagnosed to first order, using modelling results for nuclear emission regions presented in the literature.

The collective behavior of line luminosities and line ratios of the low-density and high-density tracers presents a consistent picture of the molecular medium in the nuclear regions of ULIRGs. The high-density tracers represent the molecular medium in the regions where star formation is taking place, and the low-density tracer represents the relatively unperturbed larger-scale molecular environment. The tracer luminosities increase roughly linearly with FIR luminosity but they show significant scatter in data points due to physical processes in the nuclear region, the chemical history, and the relative age of the nuclear activity. The luminosity of high-density tracers varies linearly with $L_{\mathrm{FIR}}$ except for the $\mathrm{CS}(3-2)$ luminosity, where a steeper relation is suggestive of a different excitation dependence. The $\mathrm{CO}(1-0)$ and $\mathrm{CO}(2-1)$ lines for these high FIR luminosity sources have a slope less than unity and are (almost) consistent with empirical evidence on the relation of SFR versus gas content (Kennicutt 1998).

First order diagnostics of the nuclear ISM can be based on the collective behavior of high-density tracers HCN, HNC and $\mathrm{HCO}^{+}$showing significant differentiation and systematic changes. The line strengths of these three molecules relative to the $\mathrm{CO}(1-0)$ line show a large variation and a distinct dependence on $L_{\mathrm{FIR}}$. This variation has been interpreted in terms of an evolutionary model where the depletion of molecular gas depends on the consumption and destruction of the high-density gas by the ongoing star formation process. Only partial diagnostics could be done using the available $\mathrm{CN}$ and $\mathrm{CS}$ data, which complements the diagnostics of the three other high-density tracers.

The emission line ratios of the three high-density tracers of the galaxies show a structured distribution that fills selected parts of the parameter space. Interpreting these distributions has been done using modelling column densities that are characteristic of PDR- and XDR-dominated nuclear environments. $\mathrm{OH}$ MM and other powerful ULIRGs are mostly characterized by PDR-dominance. The other end of the data distribution is characterized by mixed PDR-XDR and XDR-dominated environments. (U)LIRGs represent the phases of nuclear evolution that generate the highest FIR luminosities and most rapidly deplete the dense molecular component. Using the HCN/CO ratio as an evolutionary indicator, the distributions of data points may reflect evolution from PDR-like to more XDR-like nuclear ISM properties during the course of the outburst.

The $\mathrm{HCO}^{+} / \mathrm{HCN}$ and $\mathrm{HNC} / \mathrm{HCN}$ ratios serve as indicators of environments affected by star-formation feedback with shocks and non-standard heating and with $\mathrm{HNC}$ depletion and $\mathrm{HCO}^{+}$ enhancement in a fraction of the sources. The $\mathrm{HCO}^{+} / \mathrm{HCN}$ ratio also serves as a density indicator and would be higher under PDR circumstances and lower in XDR environments. The observed trends in the $\mathrm{HCO}^{+} / \mathrm{HCN}$ ratio could result from the (indirect) influence of an AGN in the nucleus. More likely the trends in the relative abundance of $\mathrm{HCN}$ as compared to other constituents result from evolution of the nuclear environment during the SBN activity.

The detailed interpretations of multi-line multi-molecule emission line behavior and their relation to the local environment require detailed modelling of the physical parameters of the environment together with the excitation, the chemistry, and the radiative transfer for the molecular constituents. Hereby the integration of higher level transitions of the molecules is needed to determine specific excitation temperatures and densities. A comparison with the properties of Galactic emission regions will emphasize the effect of scale size on the line ratios for tracer molecules. Further studies are underway to connect the FIR signature and global heating scenario of ULIRGs (Loenen et al. 2006) with modelling of the molecular emissions under varying conditions in a nuclear starburst. The emission scenarios for XDRs and PDRs establish the connection between star formation and other sources of excitation and the integrated emission line parameters observed in extragalactic sources.

Acknowledgements. The authors thank the IRAM staff at Pico Veleta and the staff of the Onsala Space Observatory at the SEST telescope for their valuable help during the observations. WAB thanks Wolfram Freudling for support during the startup of the SEST project and Lydia Haitas Baan for support with the observations during the second SEST run. WAB also thanks Arecibo REU summer student Melissa Nysewander (Univ. of North Carolina) for support during data reduction. The authors thank Marco Spaans for consultations on modelling aspects. This research has made use of the NASA/IPAC Extragalactic Database (NED), which is operated by the Jet Propulsion Laboratory, Caltech, under contract with NASA. This research has also made use of NASA's Astrophysics Data System Abstract Service. The authors thank the referee Melanie Krips for constructive comments and critical reading of the paper.

\section{References}

Aalto, S., Polatidis, A. G., Hüttemeister, S., \& Curran, S. J. 2002, A\&A, 381, 783

Aalto, S., Spaans, M., Wiedner, M. C., \& Hüttemeister, S. 2007, A\&A, 464, 193 Araya, E., Baan, W. A., \& Hofner, P. 2004, ApJS, 154, 541

Baan, W. A. 1989, ApJ, 338, 804

Baan, W. A., Hagiwara, Y., \& Hofner, P. 2007, ApJ, 661, 173

Baan, W. A., Haschick, A. D., \& Uglesich, R. 1993, ApJ, 415, 140

Baan, W. A., \& Klöckner, H.-R. 2006, A\&A, 449, 559

Bally, J., Stark, A. A., Wilson, R. W., \& Henkel, C. 1988, ApJ, 324, 223

Bevington, P. R., \& Robinson, D. K. 1992, Data reduction and error analysis for the physical sciences (New York: McGraw-Hill), 2nd edn.

Boger, G. I., \& Sternberg, A. 2005, ApJ, 632, 302

Braine, J., \& Combes, F. 1992, A\&A, 264, 433

Churchwell, E., Nash, A. G., \& Walmsley, C. M. 1984, ApJ, 287, 681

Dahmen, G., Huttemeister, S., Wilson, T. L., \& Mauersberger, R. 1998, A\&A, 331,959

Dickinson, D. F., Dinger, A. S. C., Kuiper, T. B. H., \& Rodriguez Kuiper, E. N. 1980, ApJ, 237, L43

Downes, D. 1989, Evolution of Galaxies: Astronomical Observations, LNP, 333, 351

Downes, D., \& Solomon, P. M. 1998, ApJ, 507, 615

Elitzur, M. 1983, ApJ, 267, 174

Fuente, A., García-Burillo, S., Gerin, M., et al. 2005, ApJ, 619, L155

Gao, Y., \& Solomon, P. M. 2004a, ApJS, 152, 63

Gao, Y., \& Solomon, P. M. 2004b, ApJ, 606, 271

Garay, G., Mardones, D., \& Mirabel, I. F. 1993, A\&A, 277, 405

García-Burillo, S., Martín-Pintado, J., Fuente, A., Usero, A., \& Neri, R. 2002, ApJ, 575, L55

Genzel, R., Lutz, D., Sturm, E., et al. 1998, ApJ, 498, 579

Goldsmith, P. F., Langer, W. D., Ellder, J., Kollberg, E., \& Irvine, W. 1981, ApJ, 249,524

Graciá-Carpio, J., García-Burillo, S., Planesas, P., \& Colina, L. 2006, ApJ, 640, L135

Graedel, T. E., Langer, W. D., \& Frerking, M. A. 1982, ApJS, 48, 321

Greaves, J. S., \& Church, S. E. 1996, MNRAS, 283, 1179

Greve, T. R., Papadopoulos, P. P., Gao, Y., \& Radford, S. J. E. 2006, ArXiv Astrophysics e-prints

Henkel, C., Baan, W. A., \& Mauersberger, R. 1991, A\&A Rev., 3, 47

Henkel, C., Mauersberger, R., Wiklind, T., et al. 1993, A\&A, 268, L17

Henkel, C., Schilke, P., \& Mauersberger, R. 1988, A\&A, 201, L23

Henkel, C., \& Wilson, T. L. 1990, A\&A, 229, 431

Hüttemeister, S., Henkel, C., Mauersberger, R., et al. 1995, A\&A, 295, 571

Iono, D., Wilson, C. D., Takakuwa, S., et al. 2007, ApJ, 659, 283

Irvine, W. M., Goldsmith, P. F., \& Hjalmarson, A. 1987, in Interstellar Processes, ed. D. J. Hollenbach, \& H. A. Thronson, Jr., ASSL, 134, 561 
Jansen, D. J., Spaans, M., Hogerheijde, M. R., \& van Dishoeck, E. F. 1995a, A\&A, 303, 541

Jansen, D. J., van Dishoeck, E. F., Black, J. H., Spaans, M., \& Sosin, C. 1995b, A\&A, 302, 223

Kennicutt, Jr., R. C. 1998, ApJ, 498, 541

Kohno, K., Matsushita, S., Vila-Vilaró, B., et al. 2001, in The Central Kiloparsec of Starbursts and AGN: The La Palma Connection, ed. J. H. Knapen, J. E. Beckman, I. Shlosman, \& T. J. Mahoney, ASP Conf. Ser., 249, 672

Krolik, J. H., \& Kallman, T. R. 1983, ApJ, 267, 610

Krumholz, M. R., \& Thompson, T. A. 2007, ArXiv e-prints, 704

Lepp, S., \& Dalgarno, A. 1996, A\&A, 306, L21

Loenen, A. F., Baan, W. A., \& Spaans, M. 2006, A\&A, 458, 89

Maloney, P. \& Black, J. H. 1988, ApJ, 325, 389

Mauersberger, R., \& Henkel, C. 1989, A\&A, 223, 79

Mauersberger, R., Henkel, C., Whiteoak, J. B., Chin, Y.-N., \& Tieftrunk, A. R. 1996a, A\&A, 309, 705

Mauersberger, R., Henkel, C., Wielebinski, R., Wiklind, T., \& Reuter, H.-P. 1996b, A\&A, 305, 421

Mauersberger, R., Henkel, C., Wilson, T. L., \& Harju, J. 1989, A\&A, 226, L5

McQuinn, K. B. W., Simon, R., Law, C. J., et al. 2002, ApJ, 576, 274

Meijerink, R., \& Spaans, M. 2005, A\&A, 436, 397

Meijerink, R., Spaans, M., \& Israel, F. P. 2006, ApJ, 650, L103

Meijerink, R., Spaans, M., \& Israel, F. P. 2007, A\&A, 461, 793

Mirabel, I. F., Booth, R. S., Johansson, L. E. B., Garay, G., \& Sanders, D. B. 1990, A\&A, 236, 327
Nguyen-Q-Rieu, Jackson, J. M., Henkel, C., Truong, B., \& Mauersberger, R. 1992, ApJ, 399, 521

Papadopoulos, P. P. 2007, ApJ, 656, 792

Radford, S. J. E., Downes, D., \& Solomon, P. M. 1991, ApJ, 368, L15

Rodriguez-Franco, A., Martin-Pintado, J., \& Fuente, A. 1998, A\&A, 329, 1097

Sanders, D. B., \& Mirabel, I. F. 1996, ARA\&A, 34, 749

Schilke, P., Walmsley, C. M., Pineau Des Forets, G., et al. 1992, A\&A, 256, 595

Schinnerer, E., Eckart, A., Tacconi, L. J., Genzel, R., \& Downes, D. 2000, ApJ, 533, 850

Snell, R. L., Goldsmith, P. F., Erickson, N. R., Mundy, L. G., \& Evans, II, N. J. 1984, ApJ, 276, 625

Sodroski, T. J., Odegard, N., Dwek, E., et al. 1995, ApJ, 452, 262

Solomon, P. M., Downes, D., \& Radford, S. J. E. 1992, ApJ, 387, L55

Solomon, P. M., Downes, D., Radford, S. J. E., \& Barrett, J. W. 1997, ApJ, 478, 144

Spaans, M., \& Meijerink, R. 2007, ApJ, 664, L23

Strong, A. W., Bloemen, J. B. G. M., Dame, T. M., et al. 1988, A\&A, 207, 1

Tacconi, L. J., Genzel, R., Tecza, M., et al. 1999, ApJ, 524, 732

Ungerechts, H., Bergin, E. A., Goldsmith, P. F., et al. 1997, ApJ, 482, 245

Usero, A., García-Burillo, S., Fuente, A., Martín-Pintado, J., \& Rodríguez-Fernández, N. J. 2004, A\&A, 419, 897

Wang, M., Henkel, C., Chin, Y.-N., et al. 2004, A\&A, 422, 883

Wootten, A. 1981, ApJ, 245, 105

Wu, J., Evans, II, N. J., Gao, Y., et al. 2005, ApJ, 635, L173

Young, J. S., \& Scoville, N. Z. 1991, ARA\&A, 29, 581 
W. A. Baan et al.: Dense gas in luminous infrared galaxies, Online Material $p 1$

\section{Online Material}


W. A. Baan et al.: Dense gas in luminous infrared galaxies, Online Material p 2

\section{Appendix A: Display spectral data}

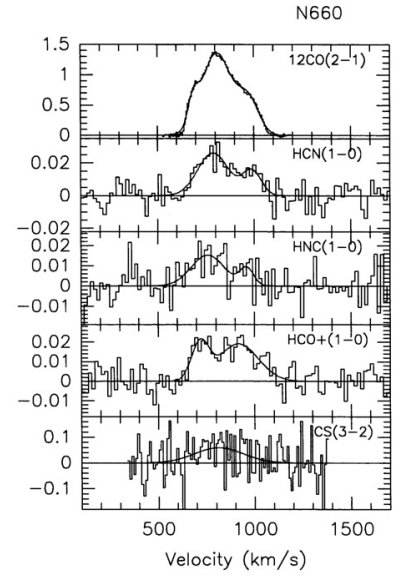

A220
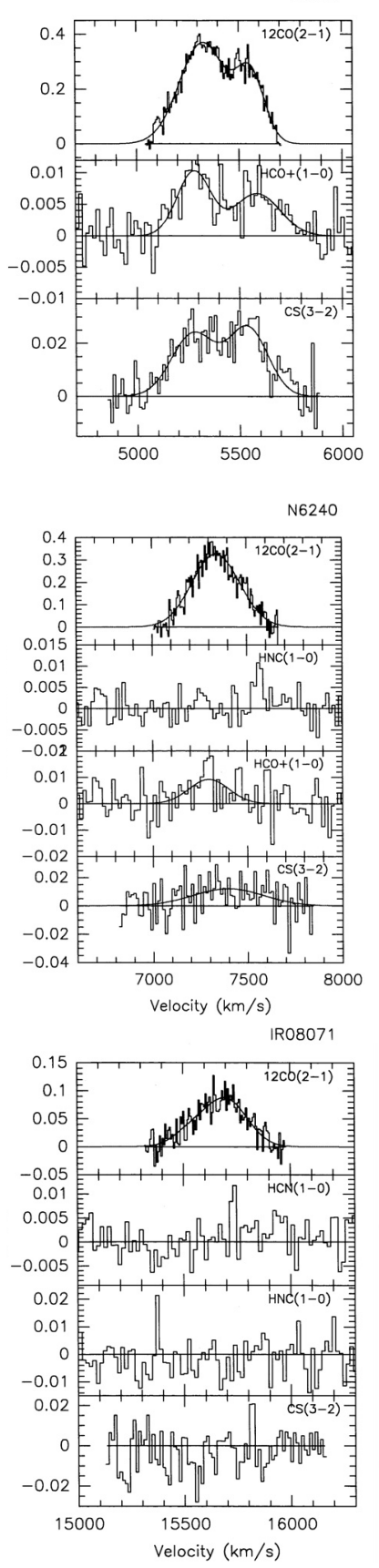
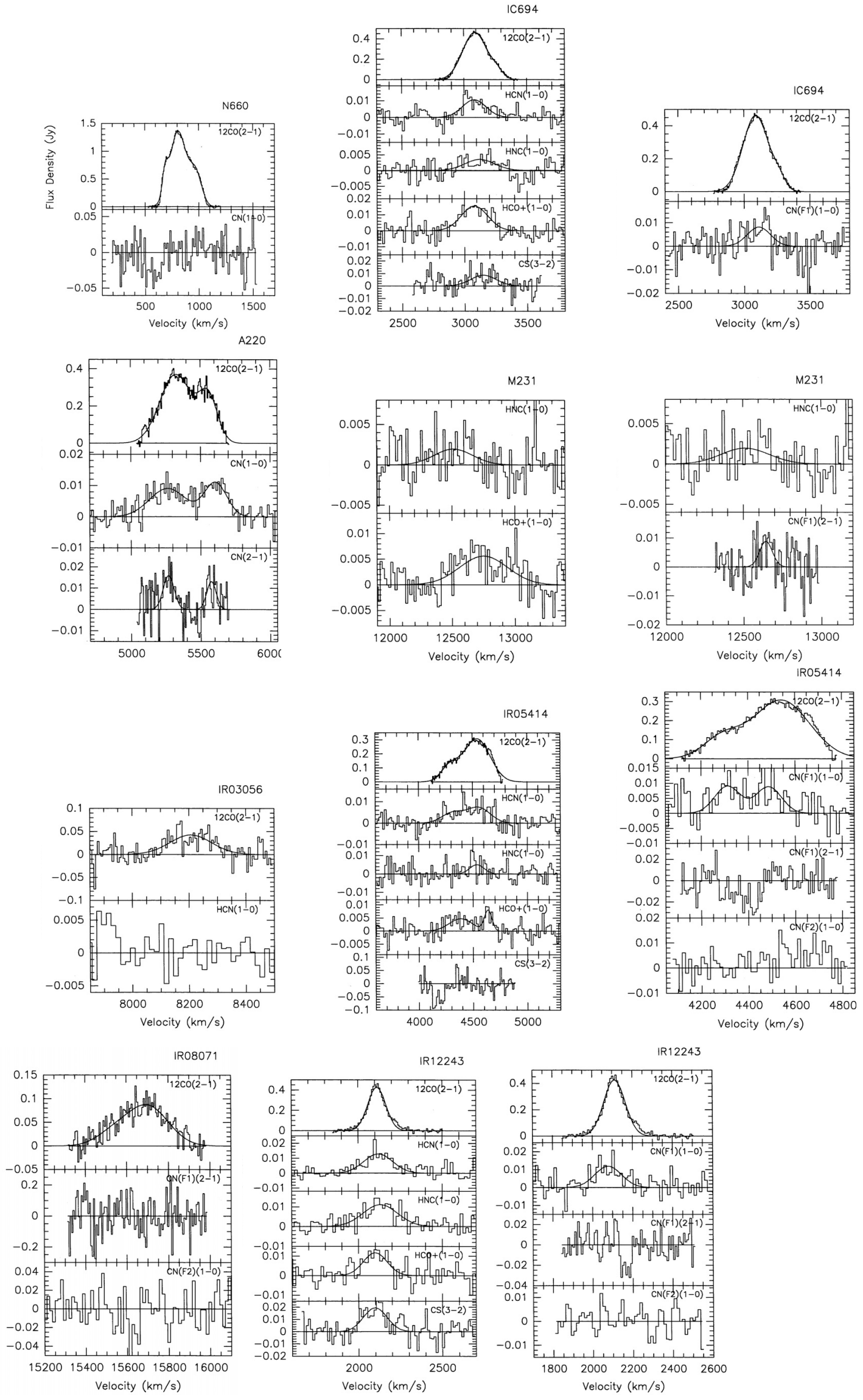

Fig. A.1. Spectra of Pico Valeta sources - Part 1. Vertical scale is $T_{\mathrm{mb}}$ in degrees. 
W. A. Baan et al.: Dense gas in luminous infrared galaxies, Online Material p 3

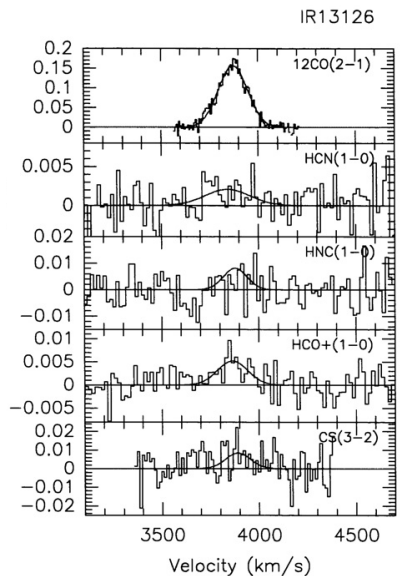

IR15107
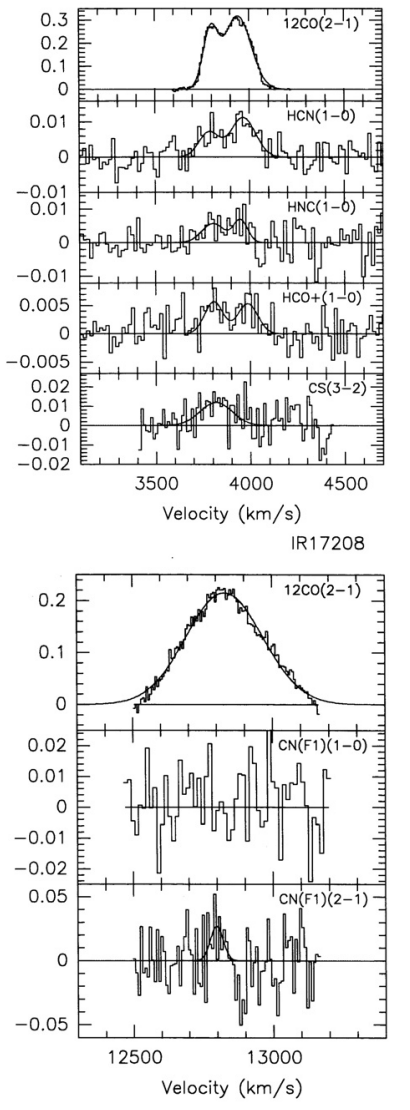

IR22025

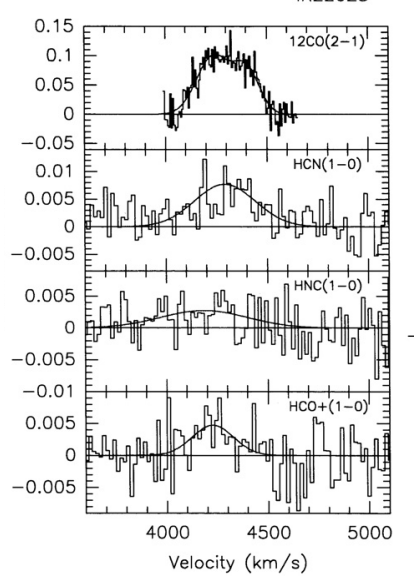

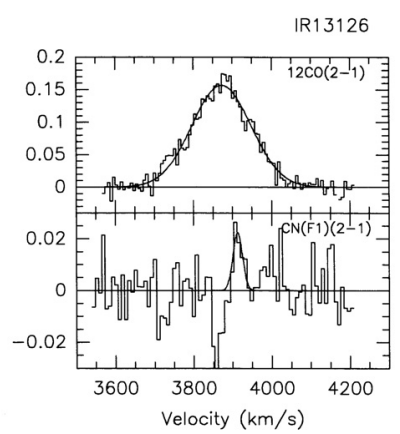

IR15107
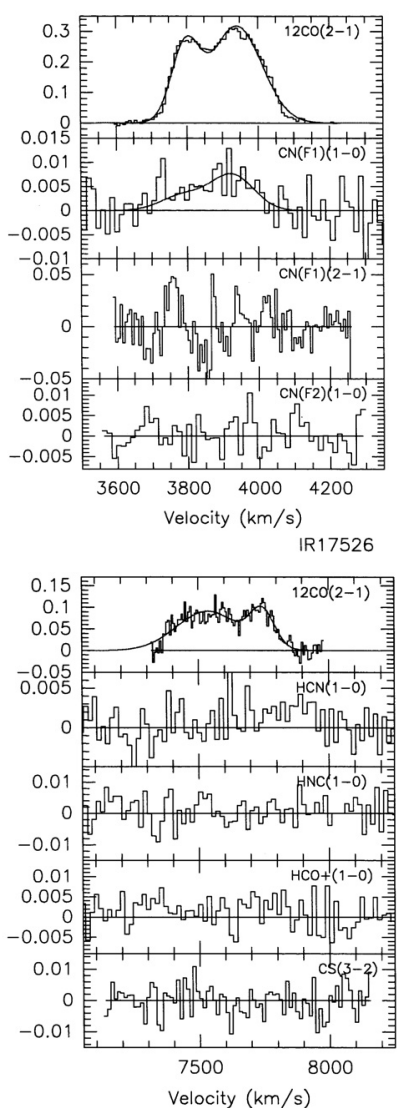

IR22025

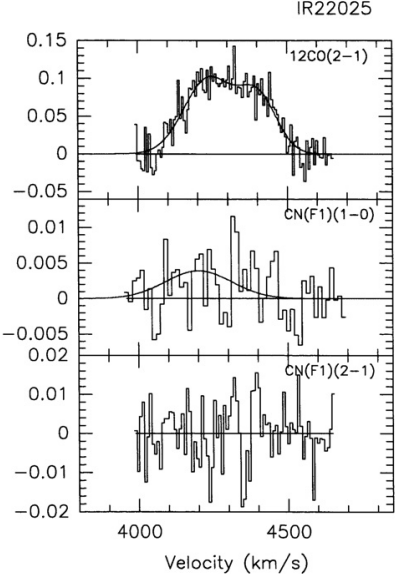

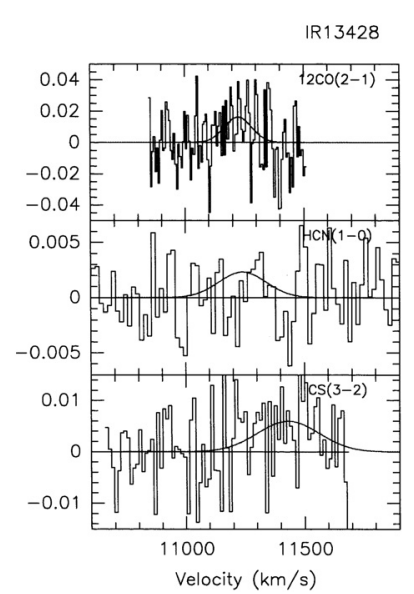
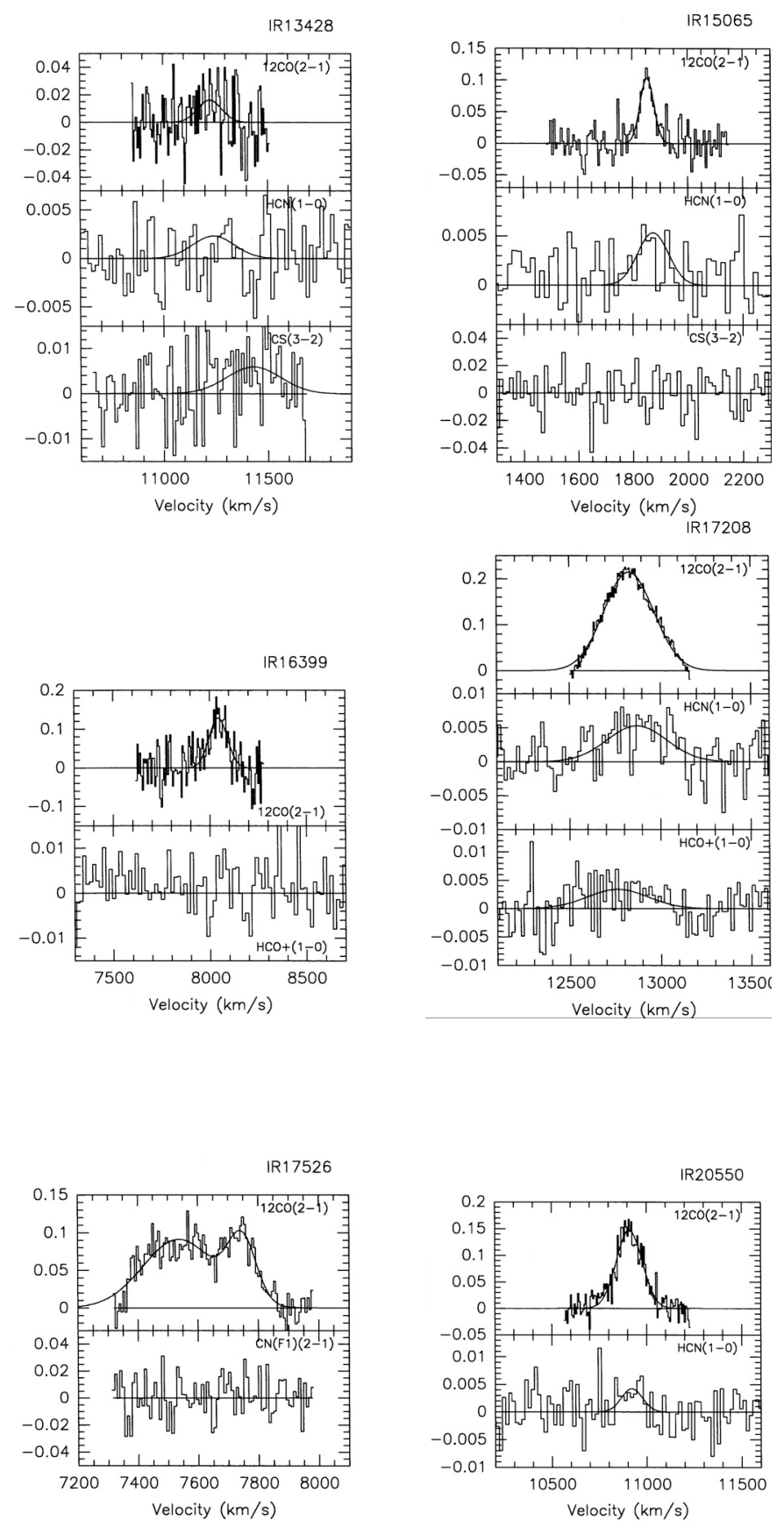

Fig. A.2. Spectra of Pico Valeta sources - Part 2. Vertical scale is $T_{\mathrm{mb}}$ in degrees. 
W. A. Baan et al.: Dense gas in luminous infrared galaxies, Online Material p 4
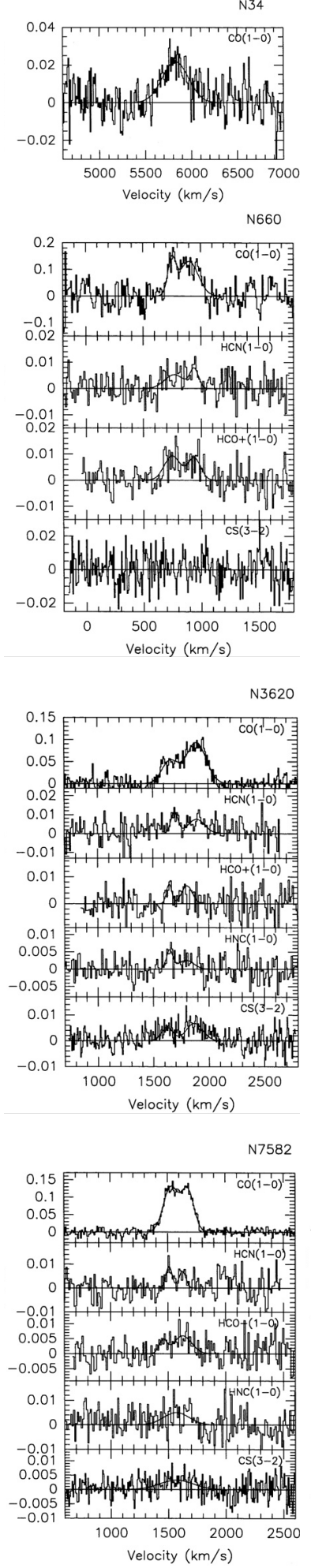
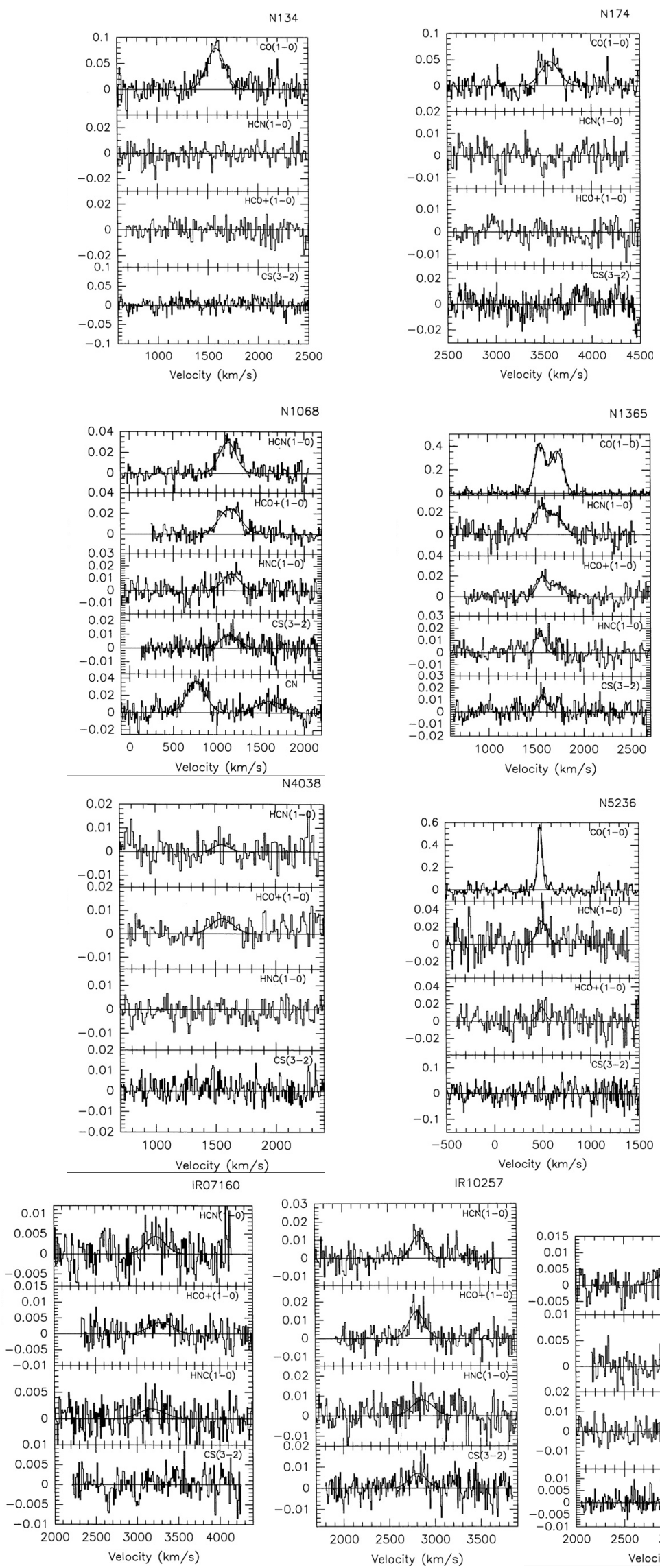
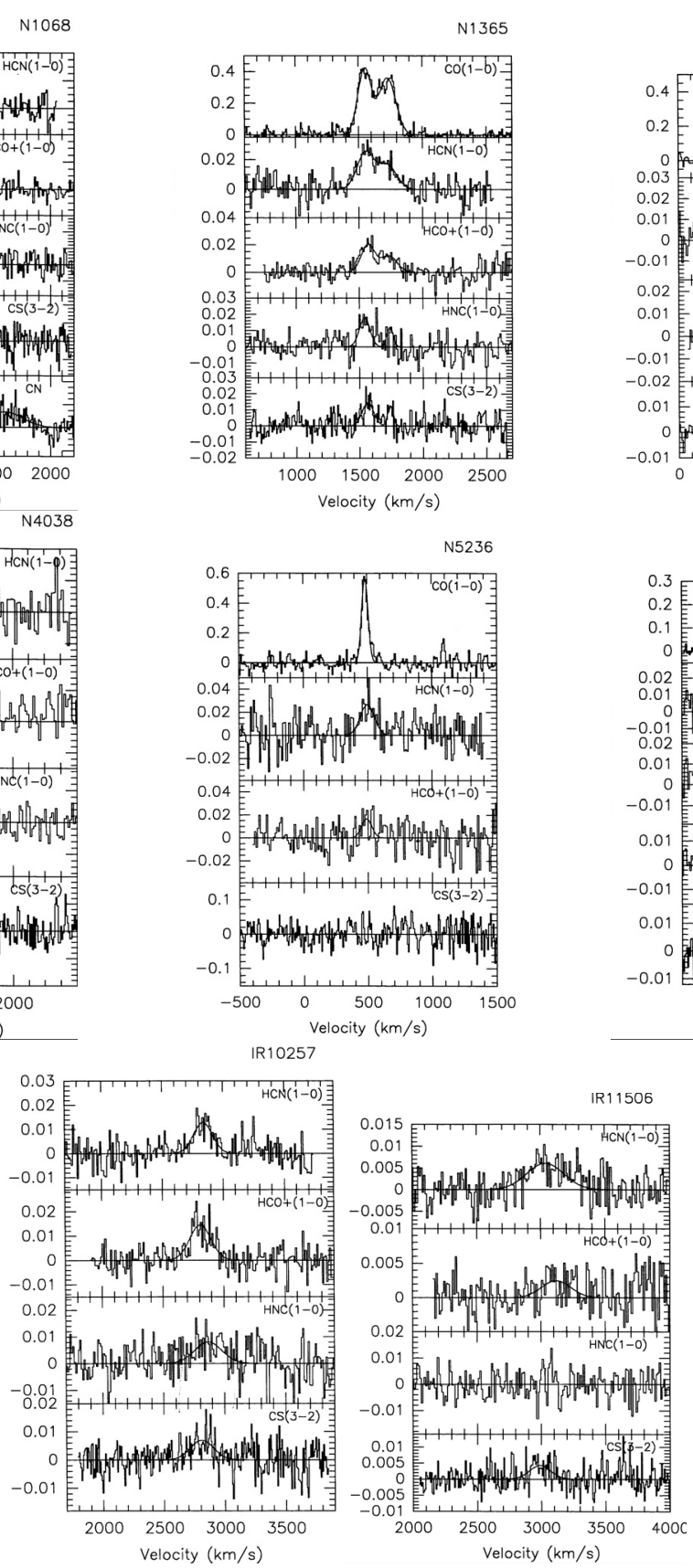

Fig. A.3. Spectra of SEST sources. Vertical scale is $T_{\mathrm{mb}}$ in degrees. Source IR 10257 is also known as M 83 or NGC 5236. 
W. A. Baan et al.: Dense gas in luminous infrared galaxies, Online Material p 5

\section{Appendix B: Data tables}

Table B.1. Observational data for High-Density Tracers based on Pico Veleta and SEST Data and from Literature .

\begin{tabular}{|c|c|c|c|c|c|c|c|c|c|c|c|c|c|c|}
\hline $\begin{array}{l}\text { Source } \\
\text { IRAS }\end{array}$ & name & MM & $\begin{array}{l}D_{\mathrm{L}}^{3} \\
\mathrm{Mpc}\end{array}$ & $\begin{array}{l}L_{\mathrm{FIR}} \\
\log \frac{L_{\mathrm{FIR}}}{L_{\odot}}\end{array}$ & $\begin{array}{l}\mathrm{CO}(1-0) \\
\mathrm{Jy} \mathrm{km} \mathrm{s}^{-1}\end{array}$ & $\begin{array}{l}\mathrm{CO}(2-1) \\
\mathrm{Jy} \mathrm{km} \mathrm{s}^{-1}\end{array}$ & $\begin{array}{l}\mathrm{HCN}(1-0) \\
\mathrm{Jy} \mathrm{km} \mathrm{s}^{-1}\end{array}$ & $\begin{array}{l}\mathrm{HNC}(1-0) \\
\mathrm{Jy} \mathrm{km} \mathrm{s}^{-1}\end{array}$ & $\begin{array}{l}\mathrm{HCO}^{+}(1-0) \\
\mathrm{Jy} \mathrm{km} \mathrm{s}^{-1}\end{array}$ & $\begin{array}{l}\mathrm{CS}(3-2) \\
\mathrm{Jy} \mathrm{km} \mathrm{s}^{-1}\end{array}$ & $\begin{array}{l}\mathrm{CN}(1-0) \\
\mathrm{Jy} \mathrm{km} \mathrm{s}^{-1}\end{array}$ & $\begin{array}{l}\mathrm{CN}(2-1) \\
\mathrm{Jy} \mathrm{km} \mathrm{s}^{-1}\end{array}$ & Telescopes & Comments \\
\hline $00085-1223$ & NGC34 & & 82.8 & 11.47 & $148.5(13.5)^{d}$ & $\ldots$ & $\ldots$ & $<7.5^{d}$ & $\cdots$ & $\ldots$ & $<8.1^{d}$ & $10.2(2.0)^{d}$ & $\overline{\mathrm{SE}}$ & - \\
\hline $00278-3331$ & NGC134 & & 22.0 & 10.60 & $336.4(27.0)$ & $\ldots$ & $<21.0$ & $\ldots$ & $<21.0$ & $<57.2$ & $\ldots$ & $\ldots$ & SE & - \\
\hline 00335-2732 & $\ldots$ & 1 & 293.5 & 11.94 & $<26.8$ & $\ldots$ & $<15.4$ & $\ldots$ & $<15.4$ & $<28.9$ & $\ldots$ & $\ldots$ & SE & - \\
\hline $00345-2945$ & NGC174 & & 49.3 & 10.92 & $211.7(19.1)$ & $\ldots$ & $<17.6$ & $\ldots$ & $<17.6$ & $<36.0$ & $\ldots$ & $\ldots$ & SE & - \\
\hline 01053-1746 & IC1623 & & 81.7 & 11.63 & $291.1(44.8)$ & $\ldots$ & $10.7^{f}$ & $\ldots$ & $12.4(2.0)$ & $\ldots$ & $\ldots$ & $\ldots$ & SE, KP & scaled value from ref. $f$ \\
\hline 01364-1042 & $\ldots$ & 1 & 203.5 & 11.85 & $46.5^{a}$ & $\ldots$ & $<6.9$ & $\ldots$ & $<6.9$ & $<14.1$ & $\ldots$ & $\ldots$ & SE & - \\
\hline $01403+1323$ & NGC660 & & 14.0 & 10.59 & $716.3(186.9)$ & $1617.3(18.4)$ & $33.0(8.3)$ & $18.9(5.7)$ & $32.8(6.3)$ & $<86.0$ & $<8.6$ & $\ldots$ & PV, SE & SEST data CO \\
\hline 02401-0013 & NGC1068 & 2 & 16.7 & 11.18 & $1902.9^{f}$ & $1967.2(82.0)^{j}$ & $157.5(12.1)$ & $61.9(8.8)$ & $143.1(9.3)$ & $50.5(6.9)$ & $285.4(24.2)$ & $\ldots$ & SE, FC, PV & $\begin{array}{l}\text { scaled refs. f \&j. PV data } \\
\text { consist. with refs. c, h \& i }\end{array}$ \\
\hline $03056+2034$ & $\ldots$ & 1 & 114.1 & 11.20 & 94.5 & 34.7 (4.2) & $<1.9$ & $\ldots$ & $\ldots$ & $\ldots$ & $\ldots$ & $\ldots$ & PV & - \\
\hline 03317-3618 & NGC1365 & & 20.8 & 11.10 & $2165.9(102.4)$ & $\ldots$ & $132.6(65.0)$ & $49.1(9.6)$ & $87.6(25.9)$ & $46.7(9.7)$ & $\ldots$ & $\ldots$ & SE & SEST data consist. with $\mathrm{f}$ \\
\hline 05059-3734 & NGC1808 & & 10.9 & 10.54 & $1897.6(137.2)$ & $\ldots$ & $107.1(5.0)$ & $45.2(10.5)$ & $59.4(3.6)$ & $10.1(3.6)$ & $102.6(2.7)^{d}$ & $82.0(4.1)^{d}$ & SE & HNC low in ref. d \\
\hline $05414+5840$ & $\ldots$ & & 62.0 & 11.25 & 411.1 & $508.0(36.6)$ & $14.9(2.5)$ & $<3.8$ & $9.1(2.6)$ & $<23.4$ & 11.6 & $<10.4$ & PV & - \\
\hline $07160-6215$ & NGC2369 & & 45.3 & 11.12 & $553.8(5.7)^{b}$ & $959.4(14.3)^{b}$ & $23.8(8.0)$ & $<13.7$ & $23.8(6.3)$ & $<20.6$ & $\ldots$ & $\ldots$ & SE & SEST data \& ref. b \\
\hline 08071+0509 & $\ldots$ & 1 & 220.1 & 11.80 & 70.9 & $115.4(7.3)$ & $<3.8$ & $<6.9$ & $\ldots$ & $<5.9$ & $\ldots$ & $<142.4$ & PV & - \\
\hline $10039-3338$ & $\ldots$ & 1 & 143.2 & 11.70 & $\ldots$ & $\ldots$ & $<9.0$ & $\ldots$ & $<9.0$ & $<13.5$ & $\ldots$ & $\ldots$ & SE & - \\
\hline $10257-4338$ & NGC3256 & & 39.3 & 11.62 & $1222.8(7.6)^{b}$ & $2980.7(14.3)^{b}$ & $46.5(8.8)$ & $<42.0$ & $59.6(6.7)$ & $<32.7$ & $32.4(1.4)^{d}$ & $22.6(2.0)^{d}$ & SE & $\mathrm{HCN}$ low in ref. $\mathrm{d}$ \\
\hline $11143-7556$ & NGC3620 & & 25.3 & 10.92 & $572.7(74.3)$ & $\ldots$ & $43.5(17.6)$ & $<15.6$ & $<23.1$ & $38.4(8.7)$ & $\ldots$ & $\ldots$ & SE & - \\
\hline $11257+5850 \mathrm{a}$ & Arp299A & 1,2 & 42.0 & 11.75 & 283.5 & $537.8(5.4)$ & $11.8(1.8)$ & $4.9(1.2)$ & $19.2(1.9)$ & $<10.4$ & $8.6(2.1)$ & $\ldots$ & PV & $\begin{array}{l}\text { IC } 694 \text {, refs. e \& f consist. } \\
\text { scaled ref. g }\end{array}$ \\
\hline $11506-3851$ & $\ldots$ & 1 & 42.8 & 11.24 & $225.3^{a}$ & $\ldots$ & $41.4(10.5)$ & $<23.1$ & $<13.3$ & $17.6(4.2)$ & $\ldots$ & $\ldots$ & SE & - \\
\hline $12018-1852$ & NGC4038 & & 22.6 & 10.69 & $\ldots$ & $\ldots$ & $8.6(5.2)$ & $<14.6$ & $26.8(6.9)$ & $<19.6$ & $\ldots$ & $\ldots$ & SE & - \\
\hline $12243-0036$ & $\ldots$ & 1 & 30.3 & 10.97 & 165.4 & $253.3(3.0)$ & $13.0(1.6)$ & $13.4(1.6)$ & $9.7(1.6)$ & $15.9(2.5)$ & $9.4(1.8)$ & $<16.1$ & PV & - \\
\hline $12540+5708$ & Mrk231 & 1 & 170.0 & 12.35 & $103.5^{g}$ & $\ldots$ & $17.6^{g}$ & $12.1(2.4)^{d}$ & $12.6(1.5)$ & $\ldots$ & $13.3(5.3)^{d}$ & $4.0(1.1)$ & OSO, PV & agrees with our data \\
\hline $13126+2452$ & $\ldots$ & & 54.3 & 11.14 & 236.2 & $131.8(2.0)$ & $<2.8$ & $<5.8$ & $4.8(1.1)$ & $<6.2$ & $\ldots$ & $3.1(1.0)$ & PV & - \\
\hline $13341-2936$ & ${\mathrm{M} 83^{4}}^{4}$ & & 3.7 & 10.07 & $653.9(33.3)$ & $\ldots$ & $68.4(21.2)$ & $20.1(1.4)^{c}$ & $30.8(12.5)$ & $5.5(1.4)^{i}$ & $\ldots$ & $\ldots$ & SE, PV & - \\
\hline $13428+5608$ & Mrk273 & 1 & 152.0 & 12.12 & $97.3^{g}$ & $\ldots$ & $15.6^{g}$ & $<6.1^{d}$ & $5.9^{g}$ & $\ldots$ & $<6.7^{d}$ & $\ldots$ & OSO & refs. g \& c consist. \\
\hline $14566-1629$ & $\ldots$ & & 48.6 & 10.64 & $<24.9$ & $\ldots$ & $\ldots$ & $\ldots$ & $\ldots$ & $<15.8$ & $\ldots$ & $\ldots$ & SE & - \\
\hline $15065-1107$ & $\ldots$ & 1 & 25.3 & 10.35 & 207.9 & $28.6(3.3)$ & $3.5(1.1)$ & $\ldots$ & $\ldots$ & $<3.4$ & $\ldots$ & $\ldots$ & PV & - \\
\hline $15107+0724$ & $\ldots$ & 1 & 47.1 & 11.14 & 118.1 & 356.3 (4.6) & $13.0(3.6)$ & $6.9(4.3)$ & $6.5(2.2)$ & $11.7(3.4)$ & $7.6(9.8)$ & $<27.4$ & PV & - \\
\hline $15327+2340$ & Arp220 & 1 & 73.0 & 12.16 & $515.0^{e}$ & $650.9(32.4)$ & $39.8^{e}$ & $34.8(7.3)^{d}$ & $18.5(3.6)$ & $57.6(12.9)$ & $68.7(10.3)^{d}$ & $39.1(12.0)^{d}$ & $\mathrm{PV}, \mathrm{OSO}, \mathrm{SE}$ & scaled values refs. f \& e \\
\hline $16399-0937$ & $\ldots$ & 1 & 111.1 & 11.50 & 118.1 & $67.4(9.2)$ & $<2.7$ & $\ldots$ & $\ldots$ & $\ldots$ & $\ldots$ & $\ldots$ & PV & - \\
\hline $16504+0228$ & NGC6240 & 2 & 98.0 & 11.76 & $333.2^{g}$ & $457.0(12.5)$ & $31.2^{g}$ & $8.0(4.0)^{d}$ & $23.4^{g}$ & $<26.3$ & $15.1(2.1)^{d}$ & $42.4(3.3)^{d}$ & SE, PV & $\begin{array}{l}\text { scaled values ref. g } \\
\text { ref. e consist. }\end{array}$ \\
\hline $17208-0014$ & $\ldots$ & 1 & 173.0 & 12.40 & $177.7^{g}$ & $327.0(3.7)$ & $12.8^{g}$ & $\ldots$ & $8.7^{g}$ & $\ldots$ & $<15.9$ & $<6.8$ & PV & - \\
\hline $17526+3253$ & $\ldots$ & 1 & 106.9 & 11.10 & 104.0 & $164.6(14.4)$ & $<3.6$ & $<8.1$ & $<6.9$ & $<8.8$ & $\ldots$ & $<26.3$ & PV & - \\
\hline $20550+1656$ & $\ldots$ & 1 & 152.7 & 11.86 & 80.3 & $111.8(4.1)$ & $2.8(1.2)$ & $\ldots$ & $\ldots$ & $\ldots$ & $\ldots$ & $\ldots$ & PV & - \\
\hline $22025+4205$ & $\ldots$ & 1 & 60.3 & 11.00 & 89.8 & $142.9(60.0)$ & $13.6(2.2)$ & $<6.9$ & $5.7(1.8)$ & $\ldots$ & $<5.2$ & $<8.2$ & PV & - \\
\hline $23134-4251$ & NGC7552 & & 22.1 & 11.02 & $652.0(88.1)$ & $\ldots$ & $34.3(6.4)$ & $16.1(5.2)$ & $30.8(6.5)$ & $22.2(4.5)$ & $\ldots$ & $\ldots$ & SE & - \\
\hline 23156-4238 & NGC7582 & & 21.9 & 10.87 & $695.5(69.0)$ & $\cdots$ & $21.2(5.8)$ & $<26.1$ & $28.3(10.3)$ & $<23.4$ & $\cdots$ & $\ldots$ & SE & - \\
\hline $00402-2350$ & $\ldots$ & & 95.0 & 11.44 & $209.8(5.7)^{b}$ & $323.9(8.2)^{b}$ & $\ldots$ & $\ldots$ & $\ldots$ & $\ldots$ & $\ldots$ & $\ldots$ & SE & - \\
\hline 00450-2533 & $\mathrm{NGC}_{25} 3^{4}$ & & 2.5 & 10.22 & $8236.0^{f}$ & $\ldots$ & $318.2(14.0)^{h}$ & $234.0(7.0)^{c}$ & $276.1(14.0)^{h}$ & $128.5(12.5)^{i}$ & $553.3(43.5)^{c}$ & $412.2(15.1)^{l}$ & PV, KP & scaled values ref. $f$ \\
\hline 01077-1707 & $\ldots$ & & 140.6 & 11.61 & $120.0^{a}$ & $\ldots$ & $\ldots$ & $\ldots$ & $\ldots$ & $\ldots$ & $\ldots$ & $\ldots$ & SE & - \\
\hline 01159-4443 & $\ldots$ & 1 & 95.7 & 11.36 & $107.8^{a}$ & $\ldots$ & $\ldots$ & $\ldots$ & $\ldots$ & $\ldots$ & $\ldots$ & $\ldots$ & SE & - \\
\hline 01219+0331 & NGC520 & & 31.6 & 10.96 & $226.8^{e}$ & $\ldots$ & $15.9^{e}$ & $\ldots$ & $\ldots$ & $\ldots$ & $\ldots$ & $\ldots$ & PV & - \\
\hline $01484+2220$ & NGC695 & & 131.0 & 11.60 & $27.3^{g}$ & $\ldots$ & $2.3^{g}$ & $\ldots$ & $3.6^{9}$ & $\ldots$ & $\ldots$ & $\ldots$ & PV & - \\
\hline $02071+3857$ & NGC 828 & & 74.6 & 11.33 & $378.0^{e}$ & $\ldots$ & $<3.7^{e}$ & $\ldots$ & $\ldots$ & $\ldots$ & $\ldots$ & $\ldots$ & PV & - \\
\hline $02114+0456$ & Mrk1027 & & 123.0 & 11.37 & $136.0^{f}$ & $\ldots$ & $4.4^{f}$ & $\ldots$ & $\ldots$ & $\ldots$ & $\ldots$ & $\ldots$ & PV & - \\
\hline $02193+4207$ & NGC891 & & 10.0 & 10.38 & $494.3^{f}$ & $381.6(26.6)^{j}$ & $8.4(2.3)^{h}$ & $4.2(0.9)^{c}$ & $9.4(3.7)^{h}$ & $\cdots$ & $\cdots$ & $\ldots$ & PV, FC & $\begin{array}{l}\text { scaled values ref. f } \\
\text { consist. with c \& h }\end{array}$ \\
\hline 02360-0653 & NGC1022 & & 21.1 & 10.39 & $337.7^{f}$ & $\ldots$ & $9.4^{f}$ & $\ldots$ & $\ldots$ & $\ldots$ & $\ldots$ & $\ldots$ & KP & - \\
\hline $02381+5923$ & Maffei2 & & 5.0 & 10.00 & $\ldots$ & $\ldots$ & $123.5(9.4)^{h}$ & $34.2(1.9)^{c}$ & $45.9(6.1)^{h}$ & $28.2(2.3)^{i}$ & $\ldots$ & $\ldots$ & PV & - \\
\hline $02391+0013$ & NGC1055 & & 14.8 & 10.29 & $2221.4^{f}$ & $2630.2(136.0)^{j}$ & $<31.3^{f}$ & $\ldots$ & $\ldots$ & $\ldots$ & $\ldots$ & $\ldots$ & KP, PV & scaled values ref. $\mathrm{j}$ \\
\hline $02526-0023$ & NGC1144 & & 118.0 & 11.39 & $216.9^{f}$ & $\ldots$ & $3.5^{f}$ & $\ldots$ & $\ldots$ & $\ldots$ & $\ldots$ & $\ldots$ & PV & - \\
\hline $03419+6756$ & IC $342^{4}$ & & 3.7 & 9.92 & $3247.2^{f}$ & $\ldots$ & $89.4(5.2)^{h}$ & $29.9(0.9)^{c}$ & $45.9(4.7)^{h}$ & $22.2(3.2)^{i}$ & $71.3(4.7)^{m}$ & $23.1(0.9)^{c}$ & PV, KP & scaled values ref. $f$ \\
\hline $04170+7510$ & NGC1530 & & 34.8 & 10.64 & $269.3^{e}$ & $\ldots$ & $9.4^{e}$ & $\ldots$ & $\ldots$ & $\ldots$ & $\ldots$ & $\ldots$ & PV & - \\
\hline 04315-0840 & NGC1614 & & 63.3 & 11.52 & $243.0(13.5)^{c}$ & $\ldots$ & $7.2^{f}$ & $\ldots$ & $\ldots$ & $\ldots$ & $16.2(8.1)^{d}$ & $20.5(4.1)^{d}$ & SE, PV & - \\
\hline 04454-4838 & $\ldots$ & 1 & 224.4 & 11.86 & $29.4^{a}$ & $\ldots$ & $\ldots$ & $\ldots$ & $\ldots$ & $\ldots$ & $\ldots$ & $\ldots$ & SE & - \\
\hline $05083+7936$ & VIIZw31 & & 220.0 & 11.92 & $100.9^{g}$ & $\cdots$ & $4.9^{g}$ & $\ldots$ & $3.5^{g}$ & $\cdots$ & $\cdots$ & $\ldots$ & PV & refs. f \& g not consist. \\
\hline $05189-2524$ & $\ldots$ & & 167.5 & 11.96 & $92.4^{f}$ & $\ldots$ & $5.3^{f}$ & $\ldots$ & $\ldots$ & $\ldots$ & $\ldots$ & $\ldots$ & $\mathrm{PV}$ & - \\
\hline $06035-7102$ & $\cdots$ & & 335.2 & 12.18 & $31.8^{a}$ & $\ldots$ & $\cdots$ & $\cdots$ & $\cdots$ & $\cdots$ & $\cdots$ & $\ldots$ & SE & - \\
\hline $06106+7822$ & NGC2146 & & 15.2 & 10.97 & $1146.4(26.7)^{e}$ & $1259.0(17.3)^{j}$ & $<18.7^{h}$ & $7.5(1.4)^{c}$ & $19.7(3.3)^{h}$ & $<9.2^{i}$ & $32.0(8.0)^{d}$ & $\ldots$ & OSO, PV & scaled values ref. $\mathrm{j}$ \\
\hline $06206-6315$ & $\ldots$ & 1 & 390.3 & 12.20 & $51.4^{a}$ & $\ldots$ & $\ldots$ & $\ldots$ & $\ldots$ & $\ldots$ & $\ldots$ & $\ldots$ & SE & - \\
\hline 06259-4708 & $\ldots$ & & 165.5 & 11.83 & $71.8(1.9)^{b}$ & $174.2(6.2)^{b}$ & $\ldots$ & $\ldots$ & $\ldots$ & $\ldots$ & $\ldots$ & $\ldots$ & SE & - \\
\hline $06592-6313$ & $\ldots$ & & 96.1 & 11.16 & $83.3^{a}$ & $\ldots$ & $\ldots$ & $\ldots$ & $\ldots$ & $\ldots$ & $\ldots$ & $\ldots$ & SE & - \\
\hline $07101+8550$ & NGC2276 & & 35.5 & 10.77 & $292.6^{f}$ & $\ldots$ & $6.8^{f}$ & $\ldots$ & $\ldots$ & $\ldots$ & $\ldots$ & $\ldots$ & KP & - \\
\hline $08354+2555$ & NGC2623 & & 76.7 & 11.56 & $346.6(13.3)^{d}$ & $\ldots$ & $\ldots$ & $14.5(3.6)^{d}$ & $\ldots$ & $\ldots$ & $<16.0^{d}$ & $\ldots$ & OSO & - \\
\hline $08520-6850$ & $\cdots$ & & 191.8 & 11.73 & $26.9^{a}$ & $\cdots$ & $\cdots$ & $\cdots$ & $\cdots$ & $\cdots$ & $\cdots$ & $\cdots$ & SE & - \\
\hline 09111-1007 & $\ldots$ & & 231.4 & 12.03 & $56.3^{a}$ & $\ldots$ & $\ldots$ & $\ldots$ & $\ldots$ & $\ldots$ & $\ldots$ & $\ldots$ & SE & - \\
\hline $09126+4432$ & Arp55 & & 161.0 & 11.68 & $48.3^{g}$ & $\ldots$ & $4.7^{g}$ & $\ldots$ & $3.9^{g}$ & $\cdots$ & $\ldots$ & $\ldots$ & PV & ref. f \& h not consist. \\
\hline $09293+2143$ & NGC2903 & & 6.2 & 9.89 & $1162.5^{f}$ & $\ldots$ & $28.1(5.6)^{h}$ & $6.5(1.4)^{c}$ & $10.3(2.8)^{h}$ & $\ldots$ & $\ldots$ & $\ldots$ & $\mathrm{PV}$ & scaled values ref. $\mathrm{f}$ \\
\hline $09320+6134$ & UGC5101 & 1 & 160.2 & 11.96 & $73.3^{f}$ & $\ldots$ & $10.4^{f}$ & $\ldots$ & $\ldots$ & $\ldots$ & $\ldots$ & $\ldots$ & PV & - \\
\hline $09517+6954$ & $\mathrm{M} 82^{4}$ & & 3.5 & 10.65 & $4579.6^{f}$ & $\ldots$ & $118.9(9.4)^{h}$ & $62.7(2.8)^{c}$ & $188.1(7.0)^{h}$ & $75.3(11.6)^{n}$ & $165.8(17.5)^{m}$ & $88.7(4.9)^{c}$ & PV, KP & scaled values ref. f \\
\hline $09585+5555$ & NGC3079 & 2 & 16.2 & 10.64 & $1768.7^{f}$ & $\ldots$ & $43.1(8.9)^{h}$ & $32.3(4.7)^{c}$ & $<9.4^{h}$ & $15.3(3.2)^{i}$ & $\ldots$ & $\ldots$ & PV, KP & scaled values ref. $\mathrm{f}$ \\
\hline $10126+7339$ & NGC3147 & & 41.6 & 10.79 & $75.6^{e}$ & $\ldots$ & $8.4^{e}$ & $\ldots$ & $\ldots$ & $\ldots$ & $\ldots$ & $\ldots$ & PV & - \\
\hline $10565+2448$ & $\ldots$ & & 173.3 & 11.99 & $77.5^{f}$ & $\ldots$ & $8.6^{f}$ & $\ldots$ & $\ldots$ & $\ldots$ & $\ldots$ & $\ldots$ & PV & - \\
\hline $11010+4107$ & Arp148 & 1 & 143.3 & 11.59 & $86.0^{f}$ & $\ldots$ & $5.4^{f}$ & $\ldots$ & $\ldots$ & $\ldots$ & $\ldots$ & $\ldots$ & PV & - \\
\hline $11085+5556$ & NGC3556 & & 10.6 & 10.11 & $1032.5^{f}$ & $\ldots$ & $16.8^{f}$ & $\ldots$ & $\ldots$ & $\ldots$ & $\ldots$ & $\ldots$ & KP & - \\
\hline $11176+1315$ & NGC3627 & & 7.6 & 10.10 & $4477.4^{f}$ & $3597.2(74.8)^{j}$ & $<21.4^{f}$ & $\ldots$ & $\ldots$ & $\ldots$ & $\ldots$ & $\ldots$ & $\mathrm{KP}, \mathrm{PV}$ & scaled values ref. $\mathrm{j}$ \\
\hline
\end{tabular}


W. A. Baan et al.: Dense gas in luminous infrared galaxies, Online Material p 6

Table B.1. continued.

\begin{tabular}{|c|c|c|c|c|c|c|c|c|c|c|c|c|c|c|}
\hline $\begin{array}{l}\text { Source } \\
\text { IRAS }\end{array}$ & name & $\overline{\mathrm{MM}}$ & $\begin{array}{l}D_{\mathrm{L}}{ }^{3} \\
\mathrm{Mpc}\end{array}$ & $\begin{array}{l}L_{\mathrm{FIR}} \\
\log \frac{L_{\mathrm{FIR}}}{L_{\odot}}\end{array}$ & $\begin{array}{l}\mathrm{CO}(1-0) \\
\mathrm{Jy} \mathrm{km} \mathrm{s}^{-1}\end{array}$ & $\begin{array}{l}\mathrm{CO}(2-1) \\
\mathrm{Jy} \mathrm{km} \mathrm{s}{ }^{-1}\end{array}$ & $\begin{array}{l}\mathrm{HCN}(1-0) \\
\mathrm{Jy} \mathrm{km} \mathrm{s}^{-1}\end{array}$ & $\begin{array}{l}\mathrm{HNC}(1-0) \\
\mathrm{Jy} \mathrm{km} \mathrm{s}^{-1}\end{array}$ & $\begin{array}{l}\mathrm{HCO}^{+}(1-0) \\
\mathrm{Jy} \mathrm{km} \mathrm{s}^{-1}\end{array}$ & $\begin{array}{l}\text { CS (3-2) } \\
\text { Jy km s s}\end{array}$ & $\begin{array}{l}\mathrm{CN}(1-0) \\
\text { Jy km s}{ }^{-1}\end{array}$ & $\begin{array}{l}\mathrm{CN}(2-1) \\
\mathrm{Jy} \mathrm{km} \mathrm{s}^{-1}\end{array}$ & Telescopes & Comments \\
\hline$\overline{11176+1351}$ & NGC3628 & & 9.6 & 10.21 & $1309.4^{f}$ & $1343.1(17.0)^{J}$ & $27.1(6.5)^{h}$ & $15.9(1.9)^{C}$ & $16.9(2.8)^{h}$ & $\ldots$ & & $\ldots$ & $\mathrm{PV}, \mathrm{KP}$ & scaled values refs. $f \& j$ \\
\hline $11257+5850 b c$ & Arp299bc & & 42.0 & 11.59 & $293.3(26.7)^{d}$ & $\ldots$ & $14.0^{e}$ & $\ldots$ & $11.1^{g}$ & $\ldots$ & $40.0(8.0)^{d}$ & $\ldots$ & OSO, PV & NGC3690, ref e consist. \\
\hline $11460+4859$ & NGC3893 & & 13.9 & 10.02 & $626.5^{f}$ & $\ldots$ & $26.0^{f}$ & $\ldots$ & $\ldots$ & $\ldots$ & $\ldots$ & $\ldots$ & KP & - \\
\hline $11578-0049$ & NGC4030 & & 17.1 & 10.31 & $1879.9^{f}$ & $\ldots$ & $39.3^{f}$ & $\ldots$ & $\ldots$ & $\ldots$ & $\ldots$ & $\ldots$ & KP & - \\
\hline $11596+6224$ & NGC4041 & & 18.0 & 10.19 & $433.6^{f}$ & $\ldots$ & $11.7^{f}$ & $\ldots$ & $\ldots$ & $\ldots$ & $\ldots$ & $\ldots$ & KP & - \\
\hline $12112+0305$ & $\ldots$ & 1 & 297.0 & 12.28 & $47.8^{9}$ & $\ldots$ & $3.6^{g}$ & $\ldots$ & $2.1^{g}$ & $\ldots$ & $\ldots$ & $\ldots$ & PV & - \\
\hline $12239+3129$ & NGC4414 & & 9.3 & 9.95 & $1982.0^{f}$ & $1501.9(68.6)^{j}$ & $38.5^{f}$ & $\ldots$ & $\ldots$ & $\ldots$ & $\ldots$ & $\ldots$ & KP, PV & scaled values ref. $\mathrm{j}$ \\
\hline $12396+3249$ & NGC4631 & & 8.1 & 10.25 & $1167.0^{f}$ & $\ldots$ & $<27.1^{f}$ & $\ldots$ & $\ldots$ & $<4.6^{i}$ & $\ldots$ & $\ldots$ & KP, PV & - \\
\hline $12542+2157$ & NGC4826 & & 4.7 & 9.44 & $2172.6^{f}$ & $\ldots$ & $<40.1^{f}$ & $\ldots$ & $\ldots$ & $\ldots$ & $\ldots$ & $\ldots$ & KP & - \\
\hline $13001-2339$ & $\ldots$ & & 90.0 & 11.45 & $142.0^{a}$ & $\ldots$ & $\ldots$ & $\ldots$ & $\ldots$ & $\ldots$ & $\ldots$ & $\ldots$ & SE & - \\
\hline $13025-4911$ & NGC4945 & & 7.8 & 11.10 & $9886.6(28.4)^{k}$ & $18878.5(12.3)^{k}$ & $420.0(7.5)^{k}$ & $225.0(4.1)^{k}$ & $450.0(3.8)^{k}$ & $275.2(4.0)^{k}$ & $756.0(18.9)^{k}$ & $602.7(8.0)^{k}$ & SE & - \\
\hline $13086+3719$ & NGC5005 & & 14.0 & 10.22 & $2542.3^{f}$ & $\ldots$ & $75.4^{f}$ & $\ldots$ & $\ldots$ & $\ldots$ & $\ldots$ & $\ldots$ & KP & - \\
\hline $13135+4217$ & NGC5055 & & 7.3 & 9.96 & $4690.5^{f}$ & $\ldots$ & $<46.5^{f}$ & $\ldots$ & $\ldots$ & $\ldots$ & $\ldots$ & $\ldots$ & KP & - \\
\hline $13183+3423$ & Arp193 & & 94.0 & 11.63 & $171.1^{g}$ & $\ldots$ & $4.9^{g}$ & $\ldots$ & $9.4^{g}$ & $\ldots$ & $\ldots$ & $\ldots$ & PV & ref. f \& e not consist. \\
\hline $13229-2934$ & NGC5135 & & 51.7 & 11.15 & $426.3^{f}$ & $\ldots$ & $21.2^{f}$ & $\ldots$ & $\ldots$ & $\ldots$ & $\ldots$ & $\ldots$ & KP & - \\
\hline $13299+4713$ & $\mathrm{M} 51^{4}$ & & 9.7 & 11.32 & $\ldots$ & $\ldots$ & $27.1(2.3)^{h}$ & $8.0(2.3)^{c}$ & $\ldots$ & $<2.3^{i}$ & $\ldots$ & $\ldots$ & PV & - \\
\hline $14306+5808$ & NGC5678 & & 27.8 & 10.44 & $811.7^{f}$ & $\ldots$ & $23.1^{f}$ & $\ldots$ & $\ldots$ & $\ldots$ & $\ldots$ & $\ldots$ & KP & - \\
\hline $14348-1447$ & $\ldots$ & & 349.5 & 12.33 & $41.6^{a}$ & $\ldots$ & $\ldots$ & $\ldots$ & $\ldots$ & $\ldots$ & $\ldots$ & $\ldots$ & SE & - \\
\hline $14376-0004$ & NGC5713 & & 24.0 & 10.58 & $505.9^{f}$ & $\ldots$ & $10.0^{f}$ & $\ldots$ & $\ldots$ & $\ldots$ & $\ldots$ & $\ldots$ & KP & - \\
\hline $14378-3651$ & $\ldots$ & & 286.2 & 12.17 & $24.5^{a}$ & $\ldots$ & $\ldots$ & $\ldots$ & $\ldots$ & $\ldots$ & $\ldots$ & $\ldots$ & SE & - \\
\hline $14514+0344$ & NGC5775 & & 21.3 & 10.57 & $868.7^{f}$ & $\ldots$ & $26.5^{f}$ & $\ldots$ & $\ldots$ & $\ldots$ & $\ldots$ & $\ldots$ & KP & - \\
\hline $16330-6820$ & $\ldots$ & & 197.8 & 11.93 & $178.8^{a}$ & $\ldots$ & $\ldots$ & $\ldots$ & $\ldots$ & $\ldots$ & $\ldots$ & $\ldots$ & SE & - \\
\hline $18093-5744$ & $\ldots$ & & 72.5 & 11.47 & $146.9^{a}$ & $\ldots$ & $\ldots$ & $\ldots$ & $\ldots$ & $\ldots$ & $\ldots$ & $\ldots$ & SE & - \\
\hline $18293-3413$ & $\ldots$ & & 72.1 & 11.73 & $686.1(7.6)^{b}$ & $1426.8(20.5)^{b}$ & $18.7^{f}$ & $\ldots$ & $\ldots$ & $\ldots$ & $\ldots$ & $\ldots$ & SE, KP & scaled values ref. $\mathrm{f}$ \\
\hline $18341-5732$ & $\ldots$ & & 64.8 & 11.26 & $257.0(3.8)^{b}$ & $307.5(12.3)^{b}$ & $\ldots$ & $\ldots$ & $\ldots$ & $\ldots$ & $\ldots$ & $\ldots$ & SE & - \\
\hline $18425+6036$ & NGC6701 & & 56.8 & 11.02 & $380.9^{f}$ & $\ldots$ & $9.0^{f}$ & $\ldots$ & $\ldots$ & $\ldots$ & $\ldots$ & $\ldots$ & KP & - \\
\hline $19115-2124$ & $\ldots$ & & 205.3 & 11.89 & $95.5^{a}$ & $\ldots$ & $\ldots$ & $\ldots$ & $\ldots$ & $\ldots$ & $\ldots$ & $\ldots$ & SE & - \\
\hline $19254-7245$ & $\ldots$ & & 260.8 & 11.96 & $58.8^{a}$ & $\ldots$ & $\ldots$ & $\ldots$ & $\ldots$ & $\ldots$ & $\ldots$ & $\ldots$ & SE & - \\
\hline $20284+2543$ & NGC6921 & & 75.5 & 11.06 & $182.0^{f}$ & $\ldots$ & $16.5^{f}$ & $\ldots$ & $\ldots$ & $\ldots$ & $\ldots$ & $\ldots$ & KP & - \\
\hline $20338+5958$ & NGC6946 & & 5.5 & 10.13 & $1432.2^{f}$ & $\ldots$ & $41.2(4.2)^{h}$ & $18.7(1.4)^{c}$ & $45.9(5.6)^{h}$ & $8.8(3.7)^{i}$ & $\ldots$ & $\ldots$ & $\mathrm{PV}, \mathrm{KP}$ & scaled values ref. $f$ \\
\hline $20511-4250$ & $\ldots$ & & 179.2 & 12.00 & $75.9^{a}$ & $\ldots$ & $\ldots$ & $\ldots$ & $\ldots$ & $\ldots$ & $\ldots$ & $\ldots$ & SE & - \\
\hline $21453-3511$ & NGC7130 & & 65.0 & 11.31 & $256.5(13.5)^{d}$ & $\ldots$ & $16.5^{f}$ & $10.0(1.2)^{d}$ & $\ldots$ & $\ldots$ & $13.5(2.7)^{d}$ & $18.4(2.0)^{d}$ & SE, KP & - \\
\hline $22132-3705$ & IC5179 & & 48.1 & 11.15 & $287.3(5.7)^{b}$ & $401.8(12.3)^{b}$ & $23.9^{f}$ & $\ldots$ & $\ldots$ & $\ldots$ & $\ldots$ & $\ldots$ & SE, KP & scaled values ref. $\mathrm{f}$ \\
\hline $22347+3409$ & NGC7331 & & 15.0 & 10.56 & $<191.6^{f}$ & $\ldots$ & $4.7(1.4)^{h}$ & $4.7(0.9)^{c}$ & $<2.8^{h}$ & $\ldots$ & $\ldots$ & $\ldots$ & $\mathrm{PV}, \mathrm{KP}$ & scaled values ref. $\mathrm{f}$ \\
\hline $22467-4906$ & $\ldots$ & & 174.7 & 11.76 & $53.9^{a}$ & $\ldots$ & $\ldots$ & $\ldots$ & $\ldots$ & $\ldots$ & $\ldots$ & $\ldots$ & SE & - \\
\hline $22491-1808$ & $\ldots$ & 1 & 329.8 & 12.16 & $22.0^{a}$ & $\ldots$ & 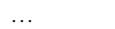 & $\ldots$ & $\ldots$ & $\ldots$ & $\ldots$ & $\ldots$ & SE & - \\
\hline $23007+0836$ & NGC7469 & & 66.0 & 11.51 & $48.1^{g}$ & $\ldots$ & $10.8^{g}$ & $17.0(2.4)^{d}$ & $15.8^{g}$ & $\ldots$ & $21.3(2.7)^{d}$ & $\ldots$ & PV, OSO & - \\
\hline $23024+1203$ & NGC7479 & & 35.2 & 10.76 & $<787.6^{f}$ & $\ldots$ & $<20.2^{f}$ & $\ldots$ & $\ldots$ & $\ldots$ & $\ldots$ & $\ldots$ & $\mathrm{KP}$ & - \\
\hline $23128-5919$ & $\ldots$ & & 186.3 & 11.98 & $46.5^{a}$ & $\ldots$ & $\ldots$ & $\ldots$ & $\ldots$ & $\ldots$ & $\ldots$ & $\ldots$ & SE & - \\
\hline $23179-6929$ & $\ldots$ & & 174.7 & 11.72 & $94.5(1.9)^{b}$ & $129.2(12.3)^{b}$ & $\ldots$ & $\ldots$ & $\ldots$ & $\ldots$ & $\ldots$ & $\ldots$ & SE & - \\
\hline $23365+3604$ & $\ldots$ & 1 & 261.0 & 12.13 & $42.1^{g}$ & $\ldots$ & $2.3^{g}$ & $\ldots$ & $1.4^{g}$ & $\ldots$ & $\ldots$ & $\ldots$ & PV & refs. f \& g not consist. \\
\hline $23488+1949$ & NGC7771 & & 57.0 & 11.32 & $496.1^{g}$ & $\ldots$ & $22.7^{9}$ & $\ldots$ & $21.5^{g}$ & $\ldots$ & $\ldots$ & $\ldots$ & PV & refs. f \& e consist. \\
\hline $23488+2018$ & Mrk331 & & 72.0 & 11.40 & $49.5^{g}$ & $\ldots$ & $8.3^{g}$ & $\ldots$ & $10.5^{g}$ & $\ldots$ & $\ldots$ & $\ldots$ & PV & refs. f \& g not consist. \\
\hline
\end{tabular}

Notes to Table 1: 1) Data without references is new data presented in this paper. References for line data from the literature: $a$ Mirabel et al. (1990), $b$ Garay et al. (1993), $c$ Hüttemeister et al. (1995),

$d$ Aalto et al. (2002), $e$ Solomon et al. (1992), $f$ Gao \& Solomon (2004a), $g$ Graciá-Carpio et al. (2006), $h$ Nguyen-Q-Rieu et al. (1992), $i$ Mauersberger et al. (1989), $j$ Braine \& Combes (1992), $k$ Wang et al. (2004), $l$ Henkel et al. (1993), $m$ Henkel et al. (1988), and $n$ Mauersberger \& Henkel (1989).

2) Column MM: 1 = OH MM, $2=\mathrm{H}_{2} \mathrm{O}$ MM

3) Distances $D_{\mathrm{L}}$ derived from radial velocities using $H_{0}=72 \mathrm{Mpc} / \mathrm{km} \mathrm{s}^{-1}$ and $q_{0}=0.5$.

4) Observational data from the literature for M 51, M 82, M 83, IC 342, NGC 253, and Maffei 2 were taken at the nuclear position using the 26" beam of the IRAM $30 \mathrm{~m}$ telescope. 
W. A. Baan et al.: Dense gas in luminous infrared galaxies, Online Material p 7

Table B.2. Line ratios for Luminous Infrared Galaxies.

\begin{tabular}{|c|c|c|c|c|c|c|c|c|c|c|c|c|}
\hline $\begin{array}{l}\text { Source } \\
\text { IRAS }\end{array}$ & name & MM & $\begin{array}{l}L_{\mathrm{FIR}} \\
\log \frac{L_{\mathrm{FIR}}}{I}\end{array}$ & $\frac{\mathrm{HCO}^{+}(1-0)}{\mathrm{HCN}(1-0)}$ & $\frac{\mathrm{HNC}(1-0)}{\mathrm{HCN}(1-0)}$ & $\frac{\mathrm{HNC}(1-0)}{\mathrm{HCO}^{+}(1-0)}$ & $\frac{\mathrm{HCN}(1-0)}{\mathrm{CO}(1-0)}$ & $\frac{\mathrm{HNC}(1-0)}{\mathrm{CO}(1-0)}$ & $\frac{\mathrm{HCO}^{+}(1-0)}{\mathrm{CO}(1-0)}$ & $\frac{\operatorname{CS}(3-2)}{\operatorname{CO}(1-0)}$ & $\frac{\mathrm{CN}(1-0)}{\mathrm{CO}(1-0)}$ & $\frac{\mathrm{CN}(2-1)}{\mathrm{CO}(1-0)}$ \\
\hline $00085-1223$ & NGC34 & & 11.47 & $\ldots$ & $\ldots$ & $\ldots$ & $\ldots$ & $<0.05$ & $\ldots$ & $\ldots$ & $<0.05$ & $0.07(0.015)$ \\
\hline $00278-3331$ & NGC134 & & 10.60 & $\ldots$ & $\ldots$ & $\ldots$ & $<0.06$ & $\ldots$ & $<0.06$ & $<0.17$ & $\ldots$ & $\ldots$ \\
\hline $00345-2945$ & NGC174 & & 10.92 & $\ldots$ & $\ldots$ & $\ldots$ & $<0.08$ & $\ldots$ & $<0.08$ & $<0.17$ & $\ldots$ & $\ldots$ \\
\hline 01053-1746 & IC1623 & & 11.63 & $1.16(0.186)$ & $\ldots$ & $\ldots$ & $0.04(0.006)$ & $\ldots$ & $0.04(0.009)$ & $\ldots$ & $\ldots$ & $\ldots$ \\
\hline 01364-1042 & $\ldots$ & 1 & 11.85 & $\ldots$ & $\ldots$ & $\ldots$ & $<0.15$ & $\ldots$ & $<0.15$ & $<0.30$ & $\ldots$ & $\ldots$ \\
\hline $01403+1323$ & NGC660 & & 10.59 & $0.99(0.315)$ & $0.58(0.224)$ & $0.58(0.206)$ & $0.05(0.017)$ & $0.03(0.010)$ & $0.05(0.015)$ & $<0.12$ & $<0.01$ & $\ldots$ \\
\hline 02401-0013 & NGC1068 & 2 & 11.18 & $0.91(0.092)$ & $0.39(0.064)$ & $0.43(0.068)$ & $0.08(0.006)$ & $0.03(0.005)$ & $0.08(0.005)$ & $0.03(0.004)$ & $0.15(0.013)$ & $\ldots$ \\
\hline 03056+2034 & $\ldots$ & 1 & 11.20 & $\ldots$ & $\ldots$ & $\ldots$ & $<0.02$ & $\ldots$ & $\ldots$ & $\ldots$ & $\ldots$ & $\ldots$ \\
\hline 03317-3618 & NGC1365 & & 11.10 & $0.66(0.378)$ & $0.37(0.196)$ & $0.56(0.199)$ & $0.06(0.030)$ & $0.02(0.005)$ & $0.04(0.012)$ & $0.02(0.005)$ & $\ldots$ & $\ldots$ \\
\hline 05059-3734 & NGC1808 & & 10.54 & $0.56(0.042)$ & $0.42(0.100)$ & $0.76(0.182)$ & $0.06(0.005)$ & $0.02(0.006)$ & $0.03(0.003)$ & $0.01(0.002)$ & $0.05(0.004)$ & $0.04(0.004)$ \\
\hline $05414+5840$ & $\ldots$ & & 11.25 & $0.61(0.204)$ & $<0.26$ & $<0.42$ & $0.04(0.006)$ & $<0.01$ & $0.02(0.006)$ & $<0.06$ & 0.03 & $<0.03$ \\
\hline $07160-6215$ & NGC2369 & & 11.12 & $1.00(0.428)$ & $<0.57$ & $<0.57$ & $0.04(0.014)$ & $<0.02$ & $0.04(0.011)$ & $<0.04$ & $\ldots$ & $\ldots$ \\
\hline $08071+0509$ & $\ldots$ & 1 & 11.80 & $\ldots$ & 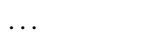 & 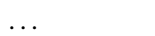 & $<0.05$ & $<0.10$ & $\ldots$ & $<0.08$ & 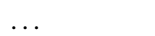 & $<2.01$ \\
\hline $10257-4338$ & NGC3256 & & 11.62 & $1.28(0.283)$ & $<0.90$ & $<0.70$ & $0.04(0.007)$ & $<0.03$ & $0.05(0.006)$ & $<0.03$ & $0.03(0.001)$ & $0.02(0.002)$ \\
\hline $11143-7556$ & NGC3620 & & 10.92 & $<0.53$ & $<0.36$ & $\ldots$ & $0.08(0.032)$ & $<0.03$ & $<0.04$ & $0.07(0.018)$ & $\ldots$ & $\ldots$ \\
\hline $11257+5850 \mathrm{a}$ & Arp299A & 1,2 & 11.75 & $1.63(0.300)$ & $0.42(0.119)$ & $0.26(0.066)$ & $0.04(0.006)$ & $0.02(0.004)$ & $0.07(0.007)$ & $<0.04$ & $0.03(0.008)$ & $\ldots$ \\
\hline $11506-3851$ & & 1 & 11.24 & $<0.32$ & $<0.56$ & & $0.18(0.046)$ & $<0.10$ & $<0.06$ & $0.08(0.018)$ & $\ldots$ & $\ldots$ \\
\hline $12018-1852$ & NGC4038 & & 10.69 & $3.11(2.053)$ & $<1.70$ & $<0.55$ & $\ldots$ & $\ldots$ & $\ldots$ & $\ldots$ & $\ldots$ & $\ldots$ \\
\hline $12243-0036$ & $\ldots$ & 1 & 10.97 & $0.75(0.153)$ & $1.03(0.175)$ & $1.38(0.278)$ & $0.08(0.010)$ & $0.08(0.010)$ & $0.06(0.010)$ & $0.10(0.015)$ & $0.06(0.011)$ & $<0.10$ \\
\hline $12540+5708$ & Mrk231 & 1 & 12.35 & $0.72(0.085)$ & $0.69(0.138)$ & $0.96(0.224)$ & 0.17 & $0.12(0.023)$ & $0.12(0.014)$ & $\ldots$ & $0.13(0.052)$ & $0.04(0.011)$ \\
\hline $13126+2452$ & $\ldots$ & & 11.14 & $>1.70$ & $\ldots$ & $<1.21$ & $<0.01$ & $<0.02$ & 0.02 & $<0.03$ & $\ldots$ & $0.01(0.004)$ \\
\hline $13341-2936$ & M83 & & 10.07 & $0.45(0.229)$ & $0.29(0.093)$ & $0.65(0.269)$ & $0.10(0.033)$ & $0.03(0.003)$ & $0.05(0.019)$ & $0.01(0.002)$ & $\ldots$ & $\ldots$ \\
\hline $13428+5608$ & Mrk273 & 1 & 12.12 & 0.38 & $<0.39$ & $<1.02$ & 0.16 & $<0.06$ & 0.06 & $\ldots$ & $<0.07$ & $\ldots$ \\
\hline $15065-1107$ & $\ldots$ & 1 & 10.35 & & & & $0.02(0.005)$ & & $\ldots$ & $<0.02$ & $\ldots$ & $\ldots$ \\
\hline $15107+0724$ & $\ldots$ & 1 & 11.14 & $0.50(0.223)$ & $0.53(0.363)$ & $1.06(0.752)$ & $0.11(0.031)$ & $0.06(0.036)$ & $0.06(0.019)$ & $0.10(0.029)$ & $0.06(0.083)$ & $<0.23$ \\
\hline $15327+2340$ & Arp220 & 1 & 12.16 & $0.46(0.089)$ & $0.87(0.184)$ & $1.88(0.536)$ & 0.08 & $0.07(0.014)$ & $0.04(0.007)$ & $0.11(0.025)$ & $0.13(0.020)$ & $0.08(0.023)$ \\
\hline $16399-0937$ & $\ldots$ & 1 & 11.50 & $\ldots$ & & & $<0.02$ & $\ldots$ & $\ldots$ & $\ldots$ & $\ldots$ & $\ldots$ \\
\hline $16504+0228$ & NGC6240 & 2 & 11.76 & 0.75 & $0.26(0.128)$ & $0.34(0.170)$ & 0.09 & $0.02(0.012)$ & 0.07 & $<0.08$ & $0.05(0.006)$ & $0.13(0.010)$ \\
\hline $17208-0014$ & $\ldots$ & 1 & 12.40 & 0.68 & $\ldots$ & $\ldots$ & $0.0^{\circ}$ & $\ldots$ & 0.05 & $\ldots$ & $<0.09$ & $<0.04$ \\
\hline $17526+3253$ & $\ldots$ & 1 & 11.10 & $\ldots$ & $\ldots$ & $\ldots$ & $<0.03$ & $<0.08$ & $<0.07$ & $<0.08$ & $\ldots$ & $<0.25$ \\
\hline $20550+1656$ & $\ldots$ & 1 & 11.86 & & & .. & $0.03(0.015)$ & & $\ldots$ & $\ldots$ & $\ldots$ & $\ldots$ \\
\hline $22025+4205$ & $\ldots$ & 1 & 11.00 & $0.42(0.151)$ & $<0.51$ & $<1.22$ & $0.15(0.025)$ & $<0.08$ & $0.06(0.020)$ & $\ldots$ & $<0.06$ & $<0.09$ \\
\hline $23134-4251$ & NGC7552 & & 11.02 & $0.90(0.253)$ & $0.47(0.176)$ & $0.52(0.204)$ & $0.05(0.012)$ & $0.02(0.009)$ & $0.05(0.012)$ & $0.03(0.008)$ & $\ldots$ & $\ldots$ \\
\hline $23156-4238$ & NGC7582 & & 10.87 & $1.34(0.609)$ & $<1.23$ & $<0.92$ & $0.03(0.009)$ & $<0.04$ & $0.04(0.015)$ & $<0.03$ & $\ldots$ & $\ldots$ \\
\hline $450-2533$ & NGC253 & & 10.22 & $0.87(0.058)$ & 0.74 & $0.85(0.050)$ & $0.04(0.002)$ & $0.03(0.001)$ & $0.03(0.002)$ & $0.02(0.002)$ & 0.07 ( & $0.05(0.002)$ \\
\hline $1219+0331$ & NGC520 & & 10.96 & & $\ldots$ & $\ldots$ & 0.0 & $\ldots$ & .. & $\ldots$ & $\ldots$ & $\ldots$ \\
\hline $1484+2220$ & NGC695 & & 11.60 & 1.59 & $\ldots$ & $\ldots$ & 0.0 & $\ldots$ & 0.13 & $\ldots$ & $\ldots$ & $\ldots$ \\
\hline $2071+3857$ & NGC828 & & 11.33 & $\ldots$ & $\ldots$ & $\ldots$ & $<0.01$ & $\ldots$ & $\ldots$ & $\ldots$ & $\ldots$ & $\ldots$ \\
\hline $2114+0456$ & Mrk1027 & & 11.37 & $\ldots$ & ... & $\ldots$ & 0.03 & $\ldots$ & $\ldots$ & $\ldots$ & $\ldots$ & $\ldots$ \\
\hline $2193+4207$ & NGC891 & & 10.38 & $1.11(0.541)$ & $0.50(0.178)$ & $0.45(0.206)$ & $0.02(0.005)$ & $0.01(0.002)$ & $0.02(0.008)$ & $\ldots$ & $\ldots$ & $\ldots$ \\
\hline $2360-0653$ & NGC1022 & & 10.39 & $\ldots$ & $\ldots$ & $\ldots$ & 0.03 & $\ldots$ & $\ldots$ & $\ldots$ & $\ldots$ & $\ldots$ \\
\hline $2381+5923$ & Maffei2 & & 10.00 & $0.37(0.057)$ & $0.28(0.026)$ & $0.74(0.107)$ & & $\ldots$ & $\ldots$ & $\ldots$ & $\ldots$ & $\ldots$ \\
\hline $2391+0013$ & NGC1055 & & 10.29 & $\ldots$ & $\ldots$ & $\ldots$ & $<0.0$ & $\ldots$ & $\ldots$ & $\ldots$ & $\ldots$ & $\ldots$ \\
\hline $02526-0023$ & NGC1144 & & 11.39 & $\ldots$ & $\ldots$ & 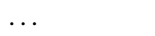 & 0.02 & & $\ldots$ & $\ldots$ & $\ldots$ & $\ldots$ \\
\hline $3419+6756$ & IC342 & & 9.92 & $0.51(0.060)$ & $0.34(0.022)$ & $0.65(0.070)$ & $0.03(0.002)$ & $0.01(0.000)$ & $0.01(0.001)$ & $0.01(0.001)$ & $0.02(0.001)$ & $0.01(0.000)$ \\
\hline $4170+7510$ & NGC1530 & & 10.64 & $\ldots$ & $\ldots$ & $\ldots$ & 0.03 & $\ldots$ & $\ldots$ & $\ldots$ & $\ldots$ & $\ldots$ \\
\hline $4315-0840$ & NGC1614 & & 11.52 & $\ldots$ & $\ldots$ & $\ldots$ & $0.03(0.002)$ & & $\ldots$ & $\ldots$ & $0.07(0.034)$ & $0.08(0.018)$ \\
\hline $05083+7936$ & VIIZw31 & & 11.92 & 0.70 & $\ldots$ & $\ldots$ & 0.05 & $\ldots$ & 0.03 & $\ldots$ & $\ldots$ & $\ldots$ \\
\hline-2524 & $\ldots$ & & 11 & $\ldots$ & $\ldots$ & $\ldots$ & 0.0 & $\ldots$ & $\ldots$ & $\ldots$ & $\ldots$ & $\ldots$ \\
\hline+7822 & NGC2146 & & 10.97 & $>1.05$ & $>0.40$ & $0.38(0.095)$ & $<0.02$ & $0.01(0.001)$ & $0.02(0$ & $<0.01$ & $0.03(0$. & $\ldots$ \\
\hline $07101+8550$ & NGC2276 & & 10.77 & $\ldots$ & $\ldots$ & $\ldots$ & 0.02 & $\ldots$ & $\ldots$ & $\ldots$ & $\ldots$ & $\ldots$ \\
\hline $4+2555$ & NGC2623 & & 11.56 & $\ldots$ & $\ldots$ & $\ldots$ & $\ldots$ & 0.04 & $\ldots$ & $\ldots$ & $<0.05$ & $\ldots$ \\
\hline $9126+4432$ & Arp55 & & 11.68 & 0.83 & .. & & 0.10 & $\cdots$ & 0.08 & $\ldots$ & $\ldots$ & $\ldots$ \\
\hline $9293+2143$ & NGC2903 & & 9.89 & $0.37(0.124)$ & $0.23(0.068)$ & $0.64(0.220)$ & $0.02(0.005)$ & $0.01(0.001)$ & $0.01(0.002)$ & $\ldots$ & $\ldots$ & $\ldots$ \\
\hline $09320+6134$ & UGC5101 & 1 & 11.96 & $\ldots$ & $\ldots$ & $\ldots$ & 0.14 & $\ldots$ & $\ldots$ & $\ldots$ & $\ldots$ & $\ldots$ \\
\hline $09517+6954$ & M82 & & 10.65 & $1.58(0.138)$ & $0.53(0.048)$ & $0.33(0.019)$ & $0.03(0.002)$ & $0.01(0.001)$ & $0.04(0.002)$ & $0.02(0.003)$ & $0.04(0.004$ & $0.02(0.001)$ \\
\hline $09585+5555$ & NGC3079 & 2 & 10.64 & $<0.22$ & $0.75(0.189)$ & $>3.45$ & $0.02(0.005)$ & $0.02(0.003)$ & $<0.01$ & $0.01(0.002)$ & $\ldots$ & $\ldots$ \\
\hline $10126+7339$ & NGC3147 & & 10.79 & $\ldots$ & $\ldots$ & $\ldots$ & 0.11 & $\ldots$ & $\ldots$ & $\ldots$ & $\ldots$ & $\ldots$ \\
\hline $10565+2448$ & $\ldots$ & & 11.99 & $\ldots$ & $\ldots$ & $\ldots$ & 0.11 & $\ldots$ & $\ldots$ & $\ldots$ & $\ldots$ & $\ldots$ \\
\hline $11010+4107$ & Arp148 & 1 & 11.59 & $\ldots$ & $\ldots$ & $\ldots$ & 0.06 & $\ldots$ & $\ldots$ & $\ldots$ & $\ldots$ & $\ldots$ \\
\hline $11085+5556$ & NGC3556 & & 10.11 & $\ldots$ & $\ldots$ & $\ldots$ & 0.02 & $\ldots$ & $\ldots$ & $\ldots$ & $\ldots$ & $\ldots$ \\
\hline $11176+1315$ & NGC3627 & & 10.10 & $\ldots$ & ... & $\ldots$ & $<0.00$ & $\ldots$ & $\ldots$ & $\ldots$ & $\ldots$ & $\ldots$ \\
\hline $11176+1351$ & NGC3628 & & 10.21 & $0.62(0.182)$ & $0.59(0.157)$ & $0.94(0.193)$ & $0.02(0.005)$ & $0.01(0.001)$ & $0.01(0$ & $\ldots$ & $\ldots$ & $\ldots$ \\
\hline $11257+5850 \mathrm{bc}$ & Arp299bc & & 11.59 & 0.79 & $\ldots$ & $\ldots$ & $0.05(0.004)$ & $\ldots$ & $0.04(0.003)$ & $\ldots$ & 0.14 & \\
\hline $11460+4859$ & NGC3893 & & 10.02 & $\ldots$ & ... & $\ldots$ & 0.04 & $\ldots$ & $\ldots$ & $\ldots$ & $\ldots$ & $\ldots$ \\
\hline
\end{tabular}


W. A. Baan et al.: Dense gas in luminous infrared galaxies, Online Material $p 8$

Table B.2. continued.

\begin{tabular}{|c|c|c|c|c|c|c|c|c|c|c|c|c|}
\hline $\begin{array}{l}\text { Source } \\
\text { IRAS }\end{array}$ & name & MM & $\begin{array}{l}L_{\mathrm{FIR}} \\
\log \frac{L_{\mathrm{FIR}}}{L_{\odot}}\end{array}$ & $\frac{\mathrm{HCO}^{+}(1-0)}{\mathrm{HCN}(1-0)}$ & $\frac{\mathrm{HNC}(1-0)}{\mathrm{HCN}(1-0)}$ & $\frac{\mathrm{HNC}(1-0)}{\mathrm{HCO}^{+}(1-0)}$ & $\frac{\mathrm{HCN}(1-0)}{\mathrm{CO}(1-0)}$ & $\frac{\mathrm{HNC}(1-0)}{\mathrm{CO}(1-0)}$ & $\frac{\mathrm{HCO}^{+}(1-0)}{\mathrm{CO}(1-0)}$ & $\frac{\mathrm{CS}(3-2)}{\mathrm{CO}(1-0)}$ & $\frac{\mathrm{CN}(1-0)}{\mathrm{CO}(1-0)}$ & $\frac{\mathrm{CN}(2-1)}{\mathrm{CO}(1-0)}$ \\
\hline $11578-0049$ & NGC4030 & & 10.31 & $\ldots$ & $\ldots$ & $\ldots$ & 0.02 & $\ldots$ & $\ldots$ & $\ldots$ & $\ldots$ & $\ldots$ \\
\hline $11596+6224$ & NGC4041 & & 10.19 & $\ldots$ & $\ldots$ & $\ldots$ & 0.03 & $\ldots$ & $\ldots$ & $\ldots$ & $\ldots$ & $\ldots$ \\
\hline $12112+0305$ & $\ldots$ & 1 & 12.28 & 0.60 & $\ldots$ & $\ldots$ & 0.07 & $\ldots$ & 0.04 & $\ldots$ & $\ldots$ & $\ldots$ \\
\hline $12239+3129$ & NGC4414 & & 9.95 & $\ldots$ & $\ldots$ & $\ldots$ & 0.02 & $\ldots$ & $\ldots$ & $\ldots$ & $\ldots$ & $\ldots$ \\
\hline $12396+3249$ & NGC4631 & & 10.25 & $\ldots$ & $\ldots$ & $\ldots$ & $<0.02$ & $\ldots$ & $\ldots$ & $<0.00$ & $\ldots$ & $\ldots$ \\
\hline $12542+2157$ & NGC4826 & & 9.44 & $\ldots$ & $\ldots$ & $\ldots$ & $<0.02$ & $\ldots$ & $\ldots$ & $\ldots$ & $\ldots$ & $\ldots$ \\
\hline $13025-4911$ & NGC4945 & & 11.10 & $1.07(0.021)$ & $0.54(0.014)$ & $0.50(0.010)$ & $0.04(0.001)$ & $0.02(0.000)$ & $0.05(0.000)$ & $0.03(0.000)$ & $0.08(0.002)$ & $0.06(0.001)$ \\
\hline $13086+3719$ & NGC5005 & & 10.22 & $\ldots$ & $\ldots$ & $\ldots$ & 0.03 & $\ldots$ & $\ldots$ & $\ldots$ & $\ldots$ & $\ldots$ \\
\hline $13135+4217$ & NGC5055 & & 9.96 & $\ldots$ & $\ldots$ & $\ldots$ & $<0.01$ & $\ldots$ & $\ldots$ & $\ldots$ & $\ldots$ & $\ldots$ \\
\hline $13183+3423$ & Arp193 & & 11.63 & 1.91 & $\ldots$ & $\ldots$ & 0.03 & $\ldots$ & 0.05 & $\ldots$ & $\ldots$ & $\ldots$ \\
\hline $13229-2934$ & NGC5135 & & 11.15 & $\ldots$ & $\ldots$ & $\ldots$ & 0.05 & $\ldots$ & $\ldots$ & $\ldots$ & $\ldots$ & $\ldots$ \\
\hline $13299+4713$ & M51 & & 11.32 & $\ldots$ & $0.29(0.090)$ & $\ldots$ & $\ldots$ & $\ldots$ & $\ldots$ & $\ldots$ & $\ldots$ & $\ldots$ \\
\hline $14306+5808$ & NGC5678 & & 10.44 & $\ldots$ & $\ldots$ & $\ldots$ & 0.03 & $\ldots$ & $\ldots$ & $\ldots$ & $\ldots$ & $\ldots$ \\
\hline $14376-0004$ & NGC5713 & & 10.58 & $\ldots$ & $\ldots$ & $\ldots$ & 0.02 & $\ldots$ & $\ldots$ & $\ldots$ & $\ldots$ & $\ldots$ \\
\hline $14514+0344$ & NGC5775 & & 10.57 & $\ldots$ & $\ldots$ & $\ldots$ & 0.03 & $\ldots$ & $\ldots$ & $\ldots$ & $\ldots$ & $\ldots$ \\
\hline $18293-3413$ & $\ldots$ & & 11.73 & $\ldots$ & $\ldots$ & $\ldots$ & $0.03(0.000)$ & $\ldots$ & $\ldots$ & $\ldots$ & $\ldots$ & $\ldots$ \\
\hline $18425+6036$ & NGC6701 & & 11.02 & $\ldots$ & $\ldots$ & $\ldots$ & 0.02 & $\ldots$ & $\ldots$ & $\ldots$ & $\ldots$ & $\ldots$ \\
\hline $20284+2543$ & NGC6921 & & 11.06 & $\ldots$ & $\ldots$ & $\ldots$ & 0.09 & $\ldots$ & $\ldots$ & $\ldots$ & $\ldots$ & $\ldots$ \\
\hline $20338+5958$ & NGC6946 & & 10.13 & $1.11(0.178)$ & $0.45(0.058)$ & $0.41(0.059)$ & $0.03(0.003)$ & $0.01(0.001)$ & $0.03(0.004)$ & $0.01(0.003)$ & $\ldots$ & $\ldots$ \\
\hline $21453-3511$ & NGC7130 & & 11.31 & $\ldots$ & $0.61(0.076)$ & $\ldots$ & $0.06(0.003)$ & $0.04(0.005)$ & $\ldots$ & $\ldots$ & $0.05(0.011)$ & $0.07(0.009)$ \\
\hline $22132-3705$ & IC5179 & & 11.15 & $\ldots$ & $\ldots$ & $\ldots$ & $0.08(0.002)$ & $\ldots$ & $\ldots$ & $\ldots$ & $\ldots$ & $\ldots$ \\
\hline $22347+3409$ & NGC7331 & & 10.56 & $<0.60$ & $1.00(0.360)$ & $>1.67$ & $>0.02$ & $>0.02$ & $\ldots$ & $\ldots$ & $\ldots$ & $\ldots$ \\
\hline $23007+0836$ & NGC7469 & & 11.51 & 1.46 & $1.57(0.224)$ & $1.07(0.153)$ & 0.22 & $0.35(0.050)$ & 0.33 & $\ldots$ & $0.44(0.055)$ & $\ldots$ \\
\hline $23365+3604$ & & 1 & 12.13 & 0.62 & $\ldots$ & $\ldots$ & 0.06 & $\ldots$ & 0.03 & $\ldots$ & $\ldots$ & $\ldots$ \\
\hline $23488+1949$ & NGC7771 & & 11.32 & 0.95 & $\ldots$ & $\ldots$ & 0.05 & $\ldots$ & 0.04 & $\ldots$ & $\ldots$ & $\ldots$ \\
\hline $23488+2018$ & Mrk331 & & 11.40 & 1.26 & $\ldots$ & $\ldots$ & 0.17 & $\ldots$ & 0.21 & $\ldots$ & $\ldots$ & $\ldots$ \\
\hline
\end{tabular}

Notes to Table 1: Column MM: $1=\mathrm{OH}$ MM, $2=\mathrm{H}_{2} \mathrm{O}$ MM. 